Universidade de São Paulo

Instituto de Astronomia, Geofísica e Ciências Atmosféricas

Departamento de Astronomia

Andressa Maria Jendreieck

\title{
Sismologia e Rotação ao Longo da Sequência \\ Principal
}

São Paulo 

Andressa Maria Jendreieck

\section{Sismologia e Rotação ao Longo da Sequência Principal}

Dissertação apresentada ao Departamento de Astronomia do Instituto de Astronomia, Geofísica e Ciências Atmosféricas da Universidade de São Paulo como parte dos requisitos para a obtenção do título de Mestre em Ciências.

Área de Concentração: Astronomia. Orientador:Prof. Dr. Eduardo Janot Pacheco.

Versão corrigida

São Paulo 
Nota: A versão original desta dissertação encontra-se disponível no Serviço de Biblioteca e Documentação do Instituto de Astronomia, Geofísica e Ciências Atmosféricas IAG/USP. 
ao infinito e além 



\section{Agradecimentos}

Ao meu orientador Eduardo Janot Pacheco e ao Laerte Andrade por toda a ajuda com o trabalho;

Ao meu pai, minha irmã, meu irmão e toda minha família, especialmente à minha mãe, que me apoiaram durante todo o Mestrado;

Aos professores que trabalham com ética e dedicação e contribuíram para minha formação;

Às minhas amigas Camila e Giovana Celinski pela amizade e apoio sempre que eu precisava;

Ao Daniel, Guilherme, Luís, Rogério e Kadu, que entraram comigo, por todas as horas de estudo e companherismo;

À Ana, Grazi, Lu e Márcia, por todas as conversas, apoio, compreensão e amizade;

À turma do café: Alberto, Chuchu, Tiago, Pedro e Phillip por todas as discussões e brincadeiras;

À turma de corrida: Tati, Mairan, Marcus e Reinaldo pela companhia;

À todos os amigos, que de uma maneira ou de outra, me ajudaram com o trabalho ou simplesmente me apoiaram;

À trilha sonora do filme 'Once' que serviu como inspiração;

À FAPESP, pelo apoio financeiro, sob o projeto $n^{o}:$ 2009/06647-0.

Esta tese/dissertação foi escrita em IATEX com a classe IAGTESE, para teses e dissertações do IAG. 

“'Have you guessed the riddle yet?' the Hatter said, turning to Alice again. 'No, I give it up,' Alice replied. 'What's the answer?' 'I haven't the slightest idea,' said the Hatter. 'Nor I,' said the March Hare. Alice sighed wearily. 'I think you might do something better with the time,' she said, 'than wasting it in asking riddles that have no answer.' 'If you knew Time as well as I do,' said the Hatter, 'you wouldn't talk about wasting it. It's him."

Lewis Carroll, Alice's Adventures in Wonderland

"Let us study stars as musical instruments."

Wasaburo Unno \& Yoji Osaki 



\section{Resumo}

A sismologia estelar oferece uma oportunidade única de sondar as propriedades internas das estrelas através do estudo de oscilações estelares. Essas oscilações são dependentes diretamente da física da cavidade onde são formadas. No entanto, a rotação da estrela introduz um eixo de simetria e levanta a degenerescência nas frequências, dificultando assim, a sua identificação. O objetivo deste trabalho foi estudar a dependência das frequências de oscilação estelar com a velocidade de rotação para modelos de massas intermediárias ao longo da sequência principal. Este estudo foi realizado através da modelagem de estrelas com uma grande variedade de massas e velocidades de rotação $\left(2-8 M_{\odot}, 20-100 \mathrm{~km} / \mathrm{s}\right)$ com os códigos CESAM/FILOU. O comportamento do splitting rotacional e de sua assimetria nos modos $g_{2}, g_{1}, p_{1}$ e $p_{2}$ mostrou uma dependência com outros parâmetros físicos, além da rotação. A assimetria apresenta variações interessantes levando a um método de diagnóstico de evolução: quando vários modos são observados, as assimetrias levam a uma determinação precisa da fase evolutiva da estrela. Modelos representativos para a estrela HD50844 foram comparados com os dados observacionais. A falta da identificação dos modos $(l, m)$ e o grande número de frequências não permite obter-se resultados precisos. 



\section{Abstract}

Asteroseismology provides a unique opportunity to probe the inner properties of stars through the study of stellar oscillations. These oscillations depend on the physics of the cavity where they are formed. However, the stellar rotation introduces an axis of symmetry and lifts the degeneracy of the frequencies, thus hindering the identification. The goal of this work was to study the dependence of the oscillation frequency on the stellar rotation velocities for models of intermediate mass along the main sequence. This study was performed through the modeling of stars with a wide range of masses and rotational velocities $\left(2.8 M_{\odot}, 20-100 \mathrm{~km} / \mathrm{s}\right)$ with the codes CESAM/FILOU. The behavior of the rotational splitting and its asymmetry for the modes $g_{2}, g_{1}, p_{1}$ and $p_{2}$ showed a dependence on other physical parameters, as well as rotation. The asymmetry presents interesting variations leading to a diagnostic method of evolution: when multiple modes are observed, the asymmetries lead to a precise determination of the evolution phase of the star. Representative models for the star HD50844 were compared with the observational data. The lack of identification of the modes $(1, \mathrm{~m})$ and the large number of frequencies does not allow to obtain accurate results. 



\section{Lista de Figuras}

1.1 Diagrama HR representando as diferentes classes de estrelas pulsantes. Créditos: Favata et al. (2000). . . . . . . . . . . . . . . . . . . . . 31

1.2 Espectro de frequências detectadas para a estrela $\delta$ Scuti FG Virginis. Créditos: Breger et al. (2005). . . . . . . . . . . . . . . . . . . .

1.3 Espectro de frequências da estrela $\beta$ Cephei HD129929. O painel superior mostra a janela espectral gerada a partir da amplitude do modo dominante e dos dados de amostragem. Créditos: Cunha et al. (2007). . . . . . . . . .

1.4 Espectro de frequências de estrelas do tipo solar, incluindo o Sol. A ordenada é arbitrária. Créditos: Cunha et al. (2007) . . . . . . . . . . . . . . . . . .

2.1 A linha contínua apresenta o comportamento da frequência de empuxo $N$ e linha tracejada, o da frequência acústica característica em termos da frequência cíclica correspondente, contra o raio fracional $r / R$, para um modelo Solar. As linha contínuas horizontais mostram as regiões de aprisionamento para um modo g com frequência $\nu=100 \mu \mathrm{Hz}$, e para um modo $\mathrm{p}$ com grau 20 e $\nu=2000 \mu H z$. Créditos: Christensen-Dalsgaard (2003). . . . 
2.2 Propagação de ondas acústicas ou de gravidade na seção transversal do interior Solar. O painel a) mostra que o caminho das ondas acústicas está ligado ao aumento da velocidade do som com a profundidade até o alcance do ponto de retorno interior (círculos pontilhados). As ondas correspondem a modos com frequência $3000 \mu \mathrm{Hz}$ e $l=75,25,20$ e 2. A linha passando pelo centro ilustra um modo radial. O painel b) mostra o caminho das ondas de gravidade correspondentes ao modo com frequência $190 \mu \mathrm{Hz}$ e $l=5$. Créditos: Cunha et al. (2007). . . . . . . . . . . . . . . .

2.3 Uma ampliação do espectro de potências de oscilações solares obtido com observações Doppler da luz integrada do disco Solar. Alguns modos foram identificados com seu grau 1 . O espectro apresenta um espaçamento regular previsto pela teoria assimptótica. A "grande" e "pequena"separação estão indicadas. Créditos: Christensen-Dalsgaard (2003). . . . . . . . . . 54

3.1 Esquema da órbita do satélite MOST com o campo de visão contínuo (CVZ). Créditos: CSA (2011). . . . . . . . . . . . . . . 65

3.2 Os campos de observação da missão CoRoT. Créditos: CNES (2011). . . . 68

3.3 Campo de visão do satélite Kepler com o esquema dos 42 detectores CCD. Créditos: NASA (2011). . . . . . . . . . . . . . . . . . . 71

4.1 Componente radial da aceleração centrífuga para os modelos de rotação diferencial $J_{L}$ (linha contínua) e rotação uniforme $J_{G}$ (linha tracejada). As quantidades apresentadas são o caso para um modelo de 1,8 $M_{\odot}$, com idade de 1, 05 Gyr e raio fotométrico de $R=2,27 R_{\odot}$. Créditos: Suárez et al. (2006). 75

5.1 Diagrama HR contendo as trajetórias evolutivas para os modelos calculados

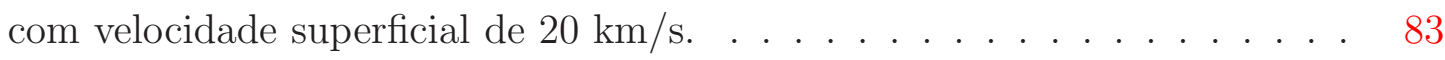

5.2 Diagrama HR contendo as trajetórias evolutivas para as massas a) $2 M_{\odot}$, b) $3 M_{\odot}$, c) $4 M_{\odot}$ e d) $5 M_{\odot}$ mostrando a influência da rotação com velocidade

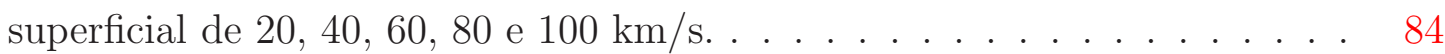

5.3 Mesmo da Figura 5.2 para as massas a) $6 M_{\odot}$, b) $7 M_{\odot}$ e c) $8 M_{\odot} . \quad \ldots \quad 85$ 
5.4 Comportamento da frequência central $(\mathrm{m}=0)$ dos modos a) $\left.\left.g_{2}, \mathrm{~b}\right) g_{1}, \mathrm{c}\right) p_{1}$ e d) $p_{2}$ em função da temperatura efetiva para as trajetórias evolutivas das massas 2 a $8 M_{\odot}$ (direita para a esquerda) mostrando a influência da rotação com velocidade superficial de 20, 40, 60, 80 e $100 \mathrm{~km} / \mathrm{s}$. . . . . . . . .

5.5 Comportamento do splitting rotacional do modo $g_{2}$ em função da temperatura efetiva para as trajetórias evolutivas das massas a) $2 M_{\odot}$, b) $3 M_{\odot}$, c) $4 M_{\odot}$ e d) $5 M_{\odot}$ mostrando a influência da rotação com velocidade superficial de 20, 40, 60, 80 e 100 km/s. Existe um problema de convergência dos códigos utilizados nos pontos à direita (picos) das Figuras b), c) e d). . . . 89

5.6 Idem a Figura 5.5, para as massas a) $6 M_{\odot}$, b) $7 M_{\odot}$ e c) $8 M_{\odot} \ldots \ldots$. . . 90

5.7 Idem a Figura 5.5, para o modo $g_{1}$ e, para as massas a) $2 M_{\odot}$, b) $3 M_{\odot}$, c) $4 M_{\odot}$ e d) $5 M_{\odot} \ldots \ldots \ldots \ldots 1$

5.8 Idem a Figura 5.7, para as massas a) $6 M_{\odot}$, b) $7 M_{\odot}$ e c) $8 M_{\odot} \ldots \ldots$. . . 92

5.9 Idem a Figura 5.5, para o modo $p_{1}$ e, para as massas a) $2 M_{\odot}$, b) $3 M_{\odot}$, c)

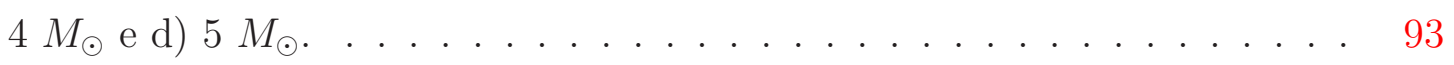

5.10 Idem a Figura 5.9 , para as massas a) $6 M_{\odot}$, b) $7 M_{\odot}$ e c) $8 M_{\odot} \ldots \ldots . .94$

5.11 Idem a Figura 5.5, para o modo $p_{2}$ e, para as massas a) $2 M_{\odot}$, b) $3 M_{\odot}$, c) $4 M_{\odot}$ e d) $5 M_{\odot} \ldots \ldots \ldots \ldots \ldots$

5.12 Idem a Figura 5.11 , para as massas a) $6 M_{\odot}$, b) $7 M_{\odot}$ e c) $8 M_{\odot} . \quad \ldots$. . 96

5.13 Comportamento do splitting rotacional dos modos a) $g_{2}$, b) $g_{1}$, c) $p_{1}$ e d) $p_{2}$ em função da temperatura efetiva para as trajetórias evolutivas das massas 2 à $8 M_{\odot}$ (direita para a esquerda) mostrando a influência da rotação com velocidade superficial de $100 \mathrm{~km} / \mathrm{s} . \ldots \ldots \ldots$

5.14 Comportamento do splitting rotacional dos modos a) $g_{2}$, b) $g_{1}$, c) $p_{1}$ e d) $p_{2}$ em função da temperatura efetiva para as trajetórias evolutivas mostrando a influência da rotação para velocidades angulares similares. A velocidade angular correspondente à $v_{r}$ ot $=20 \mathrm{~km} / \mathrm{s}$ para a $2 M_{\odot}$ é igual a $2,3 \cdot 10^{-5}$ $\mathrm{rad} / \mathrm{s}$ e à $v_{r}$ ot $=40 \mathrm{~km} / \mathrm{s}$ para $8 M_{\odot}$ é $2,1 \cdot 10^{-5} \mathrm{rad} / \mathrm{s}$. Note que a magnitude do splitting é da mesma ordem ao comparar modelos com velocidade angular próximas. 
5.15 Comportamento do assimetria do splitting rotacional do modo $g_{2}$ em função da temperatura efetiva para as trajetórias evolutivas das massas a) $2 M_{\odot}$, b) $3 M_{\odot}$, c) $4 M_{\odot}$ e d) $5 M_{\odot}$ mostrando a influência da rotação com velocidade superficial de 20, 40, 60, 80 e $100 \mathrm{~km} / \mathrm{s}$. Existe um problema de convergência dos códigos utilizados nos pontos à direita (picos) das Figuras c) e d). Note que uma ausência de assimetria pode indicar que a estrela esta numa determinada fase de evolução, onde a assimetria tende a se anular para esse modo, como p. ex., no quadro a) em $\log T_{\text {eff }} \sim 3,91 ; 3,87$ e, eventualmente $3.83 \ldots \ldots \ldots$. . . . . . . . . . . . . . . . . 100

5.16 Idem a Figura 5.15 , para as massas a) $6 M_{\odot}$, b) $7 M_{\odot}$ e c) $8 M_{\odot}$. . . . 101

5.17 Idem a Figura 5.15, para o modo $g_{1}$ e, para as massas a) $2 M_{\odot}$, b) $3 M_{\odot}$, c) $4 M_{\odot}$ e d) $5 M_{\odot}$. Note que uma ausência de assimetria pode indicar que a estrela esta numa determinada fase de evolução, onde a assimetria tende a se anular para esse modo, como p. ex., no quadro a) em $\log T_{\text {eff }} \sim 3,95 ; 3,92$ e, eventualmente $3,86 \ldots \ldots \ldots$. . . . . . . . . . . . . . . . . . . . .

5.18 Idem a Figura 5.17, para as massas a) $6 M_{\odot}$, b) $7 M_{\odot}$ e c) $8 M_{\odot}$. Note que uma ausência de assimetria pode indicar que a estrela esta numa determinada fase de evolução, onde a assimetria tende a se anular para esse modo, como p. ex., no quadro a) em $\log T_{\text {eff }} \sim 4,25 ; 4,23$ e 3.18. . . . . . . . . 103

5.19 Idem a Figura 5.15, para o modo $p_{1}$ e, para as massas a) $2 M_{\odot}$, b) $3 M_{\odot}$, c) $4 M_{\odot}$ e d) $5 M_{\odot}$. Note que uma ausência de assimetria pode indicar que a estrela esta numa determinada fase de evolução, onde a assimetria tende a se anular para esse modo, como p. ex., no quadro a) em $\log T_{\text {eff }} \sim 3,91$ e

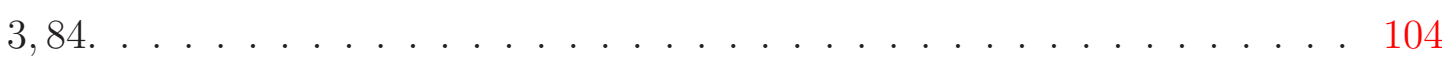

5.20 Idem a Figura 5.19, para as massas a) $6 M_{\odot}$, b) $7 M_{\odot}$ e c) $8 M_{\odot}$. . . . 105

5.21 Idem a Figura 5.15, para o modo $p_{2}$ e, para as massas a) $2 M_{\odot}$, b) $3 M_{\odot}$, c) $4 M_{\odot}$ e d) $5 M_{\odot} \ldots \ldots \ldots \ldots \ldots$ 5.22 Idem a Figura 5.21, para as massas a) $6 M_{\odot}$, b) $7 M_{\odot}$ e c) $8 M_{\odot}$. . . . 107 
5.23 Comportamento da assimetria do splitting rotacional dos modos a) $g_{2}$, b) $g_{1}$, c) $p_{1}$ e d) $p_{2}$ em função da temperatura efetiva para as trajetórias evolutivas das massas 2 a $8 M_{\odot}$ (direita para a esquerda) mostrando a influência da rotação com velocidade superficial de $100 \mathrm{~km} / \mathrm{s}$. . . . . . . . . . . . . 108

5.24 Comportamento da assimetria do splitting rotacional dos modos a) $g_{2}$, b) $g_{1}$, c) $p_{1}$ e d) $p_{2}$ em função da temperatura efetiva para as trajetórias evolutivas mostrando a influência da rotação para velocidades angulares similares. A velocidade angular correspondente à $v_{r}$ ot $=20 \mathrm{~km} / \mathrm{s}$ para a $2 M_{\odot}$ é igual a $2,3 \cdot 10^{-5} \mathrm{rad} / \mathrm{s}$ e à $v_{r}$ ot $=40 \mathrm{~km} / \mathrm{s}$ para $8 M_{\odot}$ é $2,1 \cdot 10^{-5} \mathrm{rad} / \mathrm{s}$. Note que a magnitude do splitting é da mesma ordem ao comparar modelos com velocidade angular próximas. . . . . . . . . . . . . . . . . . . 110

5.25 Espectro com as frequências obtidas por Poretti et al. (2009) da curva de luz observada com o CoRoT. Dentre essas frequências, somente 28 tiveram o valor $(1, \mathrm{~m})$ identificados através de espectroscopia. . . . . . . . . . . .

5.26 Diagrama HR contendo as trajetórias evolutivas dos 12 modelos representativos da estrela HD50844 calculados para as massas 2,0 a 2,4 $M_{\odot}$ (direita para a esquerda) com velocidade superficial de 60, 80, 100 e $120 \mathrm{~km} / \mathrm{s}$. . .

5.27 Frequência radial fundamental em função da temperatura efetiva para os 12 modelos representativos da estrela HD50844 calculados para as massas 2,0 a 2,4 $M_{\odot}$ (direita para a esquerda) com velocidade superficial de 60, 80, 100 e $120 \mathrm{~km} / \mathrm{s}$. A linha horizontal representa o valor da frequência 80,15370 $\mu H z$ obtida da observação do CoRoT por Poretti et al. (2009). . . . . . . . 115 



\section{Lista de Tabelas}

5.1 Parâmetros físicos utilizados nos modelos de equilíbrio. . . . . . . . . . . 82

5.2 Características dos modelos representativos da estrela $\delta$ Scuti HD50844. . . 109

5.3 Frequência calculada para os modos $g_{2}, g_{1}, p_{1}$ e $p_{2}$ considerando os modelos de $2,2 M_{\odot}$ dentro da caixa de erro com idade de 7,6 Gyrs. . . . . . . . . . 112 



\section{Sumário}

1. Introdução . . . . . . . . . . . . . . . . . . . . . . . . . . . 23

1.1 Perspectiva Histórica . . . . . . . . . . . . . . . . . . . . . . . . 24

1.2 Objetivo da Sismologia Estelar e Modelos Estelares . . . . . . . . . . . . . 26

1.3 Origem e Natureza Física das Oscilações . . . . . . . . . . . . . . . . . . . 28

1.4 Estrelas Pulsantes na Sequência Principal . . . . . . . . . . . . . . . . . 30

2. Pulsações Estelares . . . . . . . . . . . . . . . . . . . . . . . . . . . . . . . 35

2.1 Equações Básicas . . . . . . . . . . . . . . . . . . . . . . . 35

2.2 Equações de Oscilação . . . . . . . . . . . . . . . . . . . . . . . . . . 37

2.3 Equações de Onda nas Direções Radial e Angular . . . . . . . . . . . . . . 39

2.4 Oscilações Adiabáticas . . . . . . . . . . . . . . . . . . . . . . . . . . . 42

2.5 Propriedades dos Modos de Oscilação . . . . . . . . . . . . . . . . . . . . 44

2.5.0.1 Aproximação de Cowling . . . . . . . . . . . . . . . . . . 44

2.5.1 Aprisionamento dos Modos . . . . . . . . . . . . . . . . 45

2.5.1.1 Modos p . . . . . . . . . . . . 47

2.5.1.2 Modos g. . . . . . . . . . . . . . 48

2.6 Teoria Assimptótica . . . . . . . . . . . . . . . . . . . . . . . . . . . . . . . . . . 49

2.7 Rotação na Estrela . . . . . . . . . . . . . . . . . . . . . . 55

2.7.1 Splitting Rotacional . . . . . . . . . . . . . . 56

2.7.2 Assimetrias no Splitting Rotacional . . . . . . . . . . . . . 57

2.8 Mecanismos de Excitação das Oscilações Estelares . . . . . . . . . . . . . . 57

2.8.1 Mecanismo do tipo Motor Térmico . . . . . . . . . . . . . 57 
2.8.2 Pulsações Excitadas Estocasticamente . . . . . . . . . . . . . . . 59

2.9 Convecção . . . . . . . . . . . . . . . . . . . . . . . . . 60

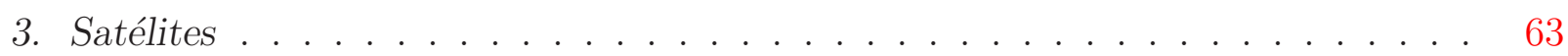

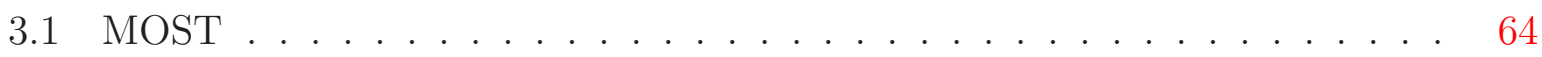

3.1 .1 Objetivo da Missão Espacial . . . . . . . . . . . . . . . . . 64

$3.1 .2 \quad$ Aspectos Gerais . . . . . . . . . . . . . . . . . . 64

3.2 CoRoT . . . . . . . . . . . . . . . . 66

3.2.1 Objetivo da Missão Espacial . . . . . . . . . . . . . . 66

3.2 .2 Aspectos Gerais . . . . . . . . . . . . . . . 67

3.3 Kepler . . . . . . . . . . . . . . . . . . . . . . . . . . 69

3.3.1 Objetivo da Missão Espacial . . . . . . . . . . . . . . . . . 69

3.3.2 Aspectos Gerais . . . . . . . . . . . . . . . . . 70

4. Modelos Estelares . . . . . . . . . . . . . . . . . . . 73

4.1 CESAM . . . . . . . . . . . . . . . . . . . . . 73

4.1 .1 Modelos de Equilíbrio . . . . . . . . . . . . . . 74

4.2 FILOU . . . . . . . . . . . . . . . . . . . 76

4.2.1 Frequências de Oscilação de um Modelo de Pseudo-Rotação . . . 78

4.2 .2 Condições de Contorno . . . . . . . . . . . . . . . . . . 79

4.2.3 Autofunções Perturbadas em Primeira Ordem . . . . . . . . . . . 79

4.2.4 Correções de Primeira e Segunda Ordem . . . . . . . . . . . . . . . 80

5. Sismologia e Rotação ao Longo da Sequência Principal . . . . . . . . . . . . . . 81

5.1 Características dos Modelos . . . . . . . . . . . . . . . 81

5.2 Efeitos da Rotação no Diagrama Hertzsprung-Russel . . . . . . . . . . . . 83

5.3 Análise do Splitting Rotacional . . . . . . . . . . . . . . . 86

5.4 Análise da Assimetria do Splitting Rotacional . . . . . . . . . . . . . . . . 97

5.5 Comparação com a estrela HD50844 . . . . . . . . . . . . . . . . . . . . 109

6. Conclusões e Perspectivas . . . . . . . . . . . . . . . . . . 117

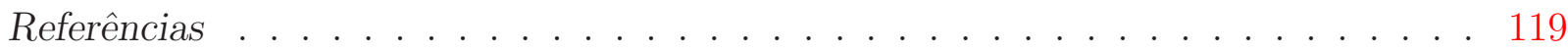


Capítulo 1

\section{Introdução}

O objetivo da astrofísica estelar é estudar a física das estrelas, sua estrutura e evolução. Numa visão geral, a teoria estelar pode ser considerada bem estabelecida já que os modelos concordam com muitas propriedades observacionais das estrelas. No entanto, ainda é necessário o conhecimento de aspectos físicos específicos para uma descrição detalhada do interior estelar. Isto é obviamente interessante, visto que as estrelas são laboratórios de física para condições extremas não atingivéis em laboratórios terrestres. Assim, dados estelares suficientemente detalhados apresentam informações sobre as propriedades da matéria nessas condições.

A maioria das observações relevantes ao interior estelar fornece apenas restrições limitadas das propriedades estelares. Se mais informações extensas estão disponíveis (como determinações detalhadas da abundância superficial), os modelos muitas vezes não conseguem explicá-las (Christensen-Dalsgaard, 2002). Aparentemente, pequenas incertezas na física de entrada dos modelos têm fortes consequências em toda a duração e no fim do ciclo de vida estelar. A falta de compreensão dos processos de transporte no interior estelar, causados por diferentes fenômenos como a rotação e a difusão magnética, é particularmente grave quando se trata de previsões precisas da evolução estelar (Aerts et al., 2008).

A heliossismologia foi um passo positivo na precisão dos modelos de estrutura interna e processos de transporte solar. A sismologia estelar visa obter avanços similares para diferentes tipos de estrela por meio da análise de suas oscilações. De fato, as oscilações estelares oferecem uma oportunidade única de colocar a prova os parâmetros de estrutura estelar e transporte internos, porque isso afeta diretamente as frequências observadas. A comparação entre as frequências medidas com alta precisão e as previstas pelos modelos 
mostra as limitações da física utilizada nos modelos e favorece a melhorias destes (Aerts et al., 2008). Logo, as observações devem abarcar uma ampla variedade de estrelas, com sensibilidade e duração suficiente para assegurar a precisão exigida (Christensen-Dalsgaard, 2010). Observações cada vez mais precisas provêm de missões espacias como MOST (Microvariability and Oscillations of STars telescope), CoRoT (Convection, Rotation and planetary Transits) e Kepler.

Esse trabalho teve como principal objetivo estudar os efeitos da rotação estelar nas frequências de oscilação para diferentes massas, com o intuito de obter as dependências e limitações das frequências com esse parâmetro físico. Para a pesquisa foram utilizados os seguintes modelos: CESAM (Morel, 1997) para obter a estrutura estelar e sua evolução e FILOU (Suárez et al., 2006) para obter as frequências de oscilação. O capítulo 2 descreve a teoria das oscilações estelares. O capítulo 3 trata das características principais dos satélites dedicados à sismologia estelar. No capítulo 4 são descritos os modelos utilizados neste trabalho. A apresentação e discussão dos resultados se encontram no capítulo 5. O capítulo 6 apresenta as conclusões e perspectivas.

\subsection{Perspectiva Histórica}

As estrelas variáveis são conhecidas desde o século XVII. Nessa época, Jan Fokkens Holwarda percebeu que a magnitude de Mira, estrela variável descoberta quase um século antes por David Fabricius, variava periodicamente em um período de 11 meses (Cunha et al., 2007). Porém, apenas quase três séculos depois entendeu-se que algumas estrelas variáveis apresentam alteração devido a pulsações intrínsecas seguindo uma sugestão de Shapley (1914), motivado pela dificuldade em se associar as variações observadas com a hipótese de binaridade. Na mesma época, o estudo das estrelas variáveis tornou-se mais sistemático. A pesquisa levou à descoberta da relação período-luminosidade de Cefeidas por Henrietta Leavitt em 1908 (Leavitt, 1908). Desde então, essas estrelas são utilizadas como "velas padrão" no universo e elas são de fundamental importância para a determinação de distâncias astrofísicas.

Depois dessa descoberta, o interesse principal das teorias de pulsação se concentrou nas Cefeidas e nas pulsações radiais. Diferentemente do progresso no desenvolvimento da teoria de pulsação radial, a teoria de pulsação não-radial permaneceu nos círculos acadêmicos 
até recentemente. Trabalhos como o de Pekeris (1938) que obteve a solução exata para oscilações não-radiais adiabáticas em um modelo compressível homogêneo e o de Cowling (1941) que estendeu o estudo para o modelo politrópico contribuíram fortemente para a divulgação do assunto.

No entanto, considera-se o início do desenvolvimento moderno da teoria de pulsação não-radial a partir do trabalho de Ledoux (1951). O autor sugeriu que oscilações nãoradiais poderiam explicar a dupla periodicidade e a grande variação temporal nas linhas espectrais de $\beta$ Canis Majoris. Osaki (1971) examinou a teoria de Ledoux ao calcular perfis de linha para uma estrela submetida a oscilação não-radial. Ele comparou o resultado com as observações disponíveis na época. Assim, ele confirmou que uma onda viajando na mesma direção da rotação explicaria a variação das linhas e as curvas de velocidade radial descontínuas.

Outra importante descoberta foram as oscilações da ordem de cinco minutos do Sol por Leighton, Noyes e Simon (1962). Muitas teorias foram propostas para explicar esse fenômeno. Deubner (1975) conseguiu resolver as oscilações observadas em modos discretos no diagrama diagnóstico que indica a natureza da oscilação ao colocar a potência observada em função do número de onda horizontal. Ele comparou-as com as previsões teóricas de Ando e Osaki (1975) e estabeleceu que as oscilações de cinco minutos do Sol eram modos-p não-radiais. O estudo das oscilações solares levou à criação de um novo campo de pesquisa conhecido como a Heliosismologia (Christensen-Dalsgaard, 2002).

Juntamente com o desenvolvimento das técnicas observacionais, que permitiram medir oscilações em diferentes tipos de estrelas, progrediu-se também em relação aos aspectos teóricos das oscilações não-radiais. Desde a metade da década de 1970, equações completas de oscilações não-radiais lineares adiabáticas e não-adiabáticas foram calculadas numericamente para modelos estelares realísticos (Unno et al., 1989). A introdução do chamado diagrama de propagação e diagrama de fase e do conceito de "aprisionamento de onda" (Scuflaire, 1974; Osaki, 1975) melhoraram enormemente o conhecimento sobre as oscilações não-radiais das estrelas.

Ao longo da sequência evolutiva de estrelas de $10 M_{\odot}$, Osaki (1975) notou um comportamento curioso dos autovetores não-radiais. Ele o interpretou como um acoplamento de oscilações de diferentes naturezas do núcleo e do envelope (Aizenman et al., 1977). 
O método assimptótico foi utilizado para esclarecer a natureza das oscilações não-radias (Wolff, 1979). Isso levou a uma fórmula analítica para as autofrequências e para os modos não-radiais de alta ordem (Tassoul, 1980).

No caso específico de alguns tipos de estrela, realizou-se progresso considerável na investigação do mecanismo de excitação dos modos. Para as Anãs Brancas, por exemplo, confirmou-se a presença de modos g instáveis produzidos pelo mecanismo $\kappa$ de opacidade das zonas de ionização de hidrogênio e hélio (e.g. Kepler et al., 1995). Já no campo da Heliossismologia, métodos de inversão foram elaborados (Gough, 1984) para obter informações do interior estelar diretamente do espectro de frequências de oscilação observado. Houve também resultados interessantes de investigações teóricas de oscilações não-radiais para estrelas com rotação (Berthomieu et al., 1978).

O século 21 trouxe desenvolvimentos importantes no campo da sismologia estelar. Com espectrógrafos altamente precisos como o CORALIE e HARPS (no European Southern Observatory/ESO-La Silla), UCLES (no ATT) e UVES (no ESO-Paranal) e com as missões espaciais MOST, CoRoT e Kepler foi possível determinar frequências de oscilação com alta precisão para diversos tipos de estrelas. O avanço nas teorias e na qualidade das observações levaram a modelos mais precisos sobre a estrutura e a evolução estelar.

\subsection{Objetivo da Sismologia Estelar e Modelos Estelares}

Os parâmetros físicos "clássicos", como temperatura efetiva, gravidade, metalicidade, não são sensíveis aos detalhes da estrutura internas das estrelas e dessa maneira não oferecem restrições suficientes para os parâmetros básicos utilizados em modelos estelares (Cunha et al., 2007). Isso afeta diretamente a qualidade dos modelos bem como o tratamento da convecção, a equação de estado, a difusão e a distribuição radial dos elementos químicos. Na falta de restrições observacionais, valores solares são utilizados, como no caso do parâmetro de comprimento de mistura, do overshooting convectivo e da difusão de elementos mais pesados. No entanto, é difícil imaginar que os mesmos parâmetros sejam adequados para diferentes tipos de estrela. Da mesma forma, nem sempre a rotação é incluída no modelo e, quando incluído, apenas com um tratamento simplificado das leis de rotação (Aerts et al., 2008).

Por outro lado, a natureza das oscilações observadas na superfície da estrela depen- 
dem da cavidade onde estas são formadas e se propagam, de modo que informações diretas da estrutura interna da estrela podem ser obtidas a partir do espectro de oscilações (Christensen-Dalsgaard, 2003). Assim, em princípio, uma medida precisa de um grande número de frequências numa estrela permite um estudo detalhado de seu interior. Além disso, restrições suficientes para determinar a localização da estrela numa elipse de erro no diagrama HR podem ser determinadas com melhor precisão (Cunha et al., 2007).

A sismologia estelar pode, então, desempenharam um papel importante no avanço da física e compreensão dos interiores estelares. Para lidar com alguns detalhes físicos desconhecidos, códigos de evolução estelar incluem parâmetros que podem ser ajustados. A maioria se refere ao tratamento de convecção, difusão, equação de estado, opacidades e balanço de elementos pesados. Um tratamento impreciso, principalmente da convecção e difusão, pode levar a importantes implicações nos modelos estelares (Cunha et al., 2007).

A maioria das estrelas apresenta instabilidades convectivas no núcleo ou no envelope. Mesmo assim, o tratamento apropriado dos modelos de convecção é algo bastante difícil (Cunha et al., 2007). O modelo de convecção mais utilizado no contexto de modelagem estelar é a Teoria do Comprimento de Mistura (Mixing Length/MLT) (Böhm-Vitense, 1958). Uma alternativa também frequentemente utilizada foi desenvolvida por Canuto e Mazzitelli (1991). Nela, correções no fluxo convectivo turubulento e uma nova expressão para a incompressibilidade livre de parâmetros ajustáveis foram incorporadas. Além da convecção, existe o problema da extensão do overshooting (e.g. Zahn, 1991). Com observações cada vez mais precisas de estrelas mais evoluídas torna-se mais importante conhecer em detalhes o overshooting nos modelos. Outro aspecto relevante diz respeito aos estudos sobre a interação da convecção com outros fenômenos físicos, como rotação, campo magnético e radiação, que apresentam fortes incertezas. Em geral, todos esses senões limitam a precisão obtida na determinação da idade e outros parâmetros globais das estrelas.

Por outro lado, a difusão de elementos químicos ocorre na maioria das estrelas e desempenha um papel importante em diversos contextos em astrofísica estelar, inclusive em estudos sobre o Sol, a maioria das estrelas na sequência principal e também para as Anãs Brancas (Cunha et al., 2007). No caso da difusão, a composição original da estrela muitas vezes não pode ser observada diretamente por ser diferente na superfície. Na verdade, há vários mecanismos físicos não muito bem entendidos mas que levariam a misturas diferentes 
na superfície e no interior. Esses mecanismos podem ser: arranjo gravitacional, levitação radiativa, mistura devido à rotação e perda de massa por vento estelar (Théado et al., 2005). Porém, para testar os modelos de difusão, é necessário conectá-los a observações que, de certa forma, só podem ser úteis se modelos precisos das camadas atmosféricas estiverem disponíveis. No entanto, estrelas que possuem o efeito de difusão mais evidente como estrelas peculiares - tendem a possuir atmosferas complexas, tornando difícil realizar testes diretos dos modelos de difusão (Cunha et al., 2007). Contudo, a sismologia estelar permite fazer testes indiretos e inferir sobre a concorrência entre a difusão e processos de mistura no interior de estrelas pulsantes. Algumas tentativas de realizar tais testes foram executadas com dados sísmicos de estrelas roAp (Cunha et al., 2004; Vauclair e Théado, 2004).

Para as estrelas em geral, o conhecimento de sua estrutura interna é limitada também pela presença eventual de agentes externos que possam introduzir desvios na simetria esférica. Os códigos de evolução estelar supõem normalmente que as estrelas apresentam simetria esférica e que alguns aspectos como o da rotação e campo magnético são geralmente ignorados em termos do interior estelar. Até recentemente, os modelos baseados em simetria esférica reproduziam razoavelmente bem as observações, mas isso mudou na última década com o desenvolvimento de novos instrumentos capazes de resolver a superfície de estrelas que não o Sol (Cunha et al., 2007). Exemplos disso são os casos onde grandes desvios da esfericidade foram detectados usando a técnica de interferometria (e.g. Domiciano de Souza et al., 2003). Essas descobertas e outras restrições para a esfericidade do interior estelar que podem ser reveladas pelas oscilações estelares motivaram o desenvolvimento de novos códigos de evolução estelar que levam em conta a simetria não esférica, particularmente causada por rotação.

\subsection{Origem e Natureza Física das Oscilações}

Para a sismologia estelar poder ajudar na compreensão do interior estelar, é necessário o conhecimento da física subjacente aos fenômenos observados. Obviamente, as oscilações dependem da física da cavidade onde elas são geradas e se propagam. Numa análise sísmica, procura-se então entender a origem e a natureza dos fenômenos físicos de que dependem as pulsações. 
Nos espectros de pulsações estelares, incluindo o do Sol, há um amplo intervalo de frequências e essas pulsações podem ter simetria esférica ou apresentarem grande variedade estrutural. Contudo, para o Sol a resolução do disco permite a observação de pequenas estruturas, enquanto que para as outras estrelas o que é observado é a estrutura em larga escala. Portanto, isso significa que pode-se detectar nas variações de brilho apenas modos de 3 ou 4 linhas nodais (Cunha et al., 2007). No caso solar, as observações resultaram na obtenção de informações com alta precisão sobre a física de seu interior como espessura da camada convectiva, perfil de rotação, variação radial da velocidade do som e abundância do Hélio (Christensen-Dalsgaard, 2002).

As pulsações ocorrem devido à presença de instabilidades na estrela. Há três tipos de estabilidades fundamentais numa estrela para que esta mantenha sua configuração de equilíbrio: as estabilidades dinâmica, vibracional e térmica. No caso de uma contração pequena e arbitrária ocorrer na estrela como um todo, o incremento do gradiente de pressão aumenta e excede o da força gravitacional, e então a estrutura em equilibrio é restaurada. Esse é o estado de estabilidade dinâmica no qual as estrelas devem passar a maior parte de suas vidas (Kippenhahn e Weigert, 1990). No entanto, instabilidades dinâmicas locais podem acontecer. De fato, a convecção nas estrelas é a manifestação de instabilidades dinâmicas diante das perturbações não-radiais. Como foi observado por Shapley (1914) no caso das Cefeidas, o tempo de escala dinâmico pode ser utilizado para estimar o período de pulsação da estrela:

$$
t_{d y n} \simeq\left(\frac{R^{3}}{G M}\right)^{1 / 2} \simeq(G \bar{\rho})^{-1 / 2},
$$

onde $R$ e $M$ são o raio e a massa da estrela respectivemente, $G$ é a constante gravitacional e $\bar{\rho}$ é a densidade média da estrela. Logo, mesmo uma simples medida do período de pulsação leva a algumas indicações sobre as propriedades gerais de uma estrela.

O equilíbrio hidrostático pode ser assumido no estudo da estabilidade térmica. Geralmente, um excesso de energia térmica causa a expansão do gás e como o teorema do virial implica, a temperatura normalmente diminui por ajustes hidrostáticos. No entanto, o equilíbrio hidrostático é restrito a perturbações de pressão e densidade com simetria esférica na ausência de campos externos e assim, as instabilidades térmicas não seriam relacionadas com pulsações não-radiais. Instabilidades não-radiais térmicas até são possíveis com o gradiente de peso molecular médio, porém nenhum exemplo desse caso foi encontrado 
(Unno et al., 1989).

Uma oscilação acontece quando um sistema dinamicamente estável sofre uma perturbação. Esta pode ser intrinsecamente estáveis ou instáveis. As oscilações intrinsecamente instáveis resultam da amplificação de perturbações menores, através de um mecanismo de motor térmico atuando numa região apropriada da estrela. A perturbação cresce até que um mecanismo qualquer estabeleça uma amplitude limite. Esse mecanismo depende de detalhes sutis do modo, e por isso a amplitude final pode ser grande para vários modos (Cunha et al., 2007). E isso também depende da localização precisa de características bem determinadas da opacidade ao longo da estrela, fazendo com que essas instabilidades estejam concentradas em regiões específicas do diagrama HR (ver seção 1.4).

As estrelas apresentam vários modos de oscilação e esses modos são caracterizados, para uma simetria esférica, por autofunções proporcionais a harmônicos esféricos (ver capítulo 2)

$$
Y_{l}^{m}(\theta, \phi),
$$

para $l=0,1,2, \ldots$ e $m=0, \pm 1, \ldots, \pm l$. As autofrequências dependem de $\mathbf{l}$ e são degeneradas por $(2 l+1)$ em $\mathbf{m}$. Além disso, os modos são distinguidos pelo número $\mathbf{n}$ que reprensenta o número de nós da componente radial. Um modo é classificado por seus números quâticos $\mathbf{n}$ e $\mathbf{l}$. A introdução de rotação ou campo magnético levanta a degenerescência em $\mathbf{m}$ e este também deve ser então especificado.

A força restauradora que causa as oscilações pode ser do tipo pressão ou gravidade. Porém, a força da gravidade, por ser radial, não pode gerar oscilações puramente radiais, mas a força de empuxo pode agir como força restauradora de oscilações não-radiais. Assim, os modos de oscilação podem ser de dois tipos: modos acústicos (modos p) ou modos de gravidade (modos g). As estrelas normalmente apresentam um dos dois tipos de oscilação, mas algumas, principalmente estrelas mais evoluídas, podem apresentar modos de natureza mista (Christensen-Dalsgaard, 2010). O Sol apresenta modos de gravidade de comprimentos de onda relativamente curtos (Christensen-Dalsgaard, 2002).

As oscilações precisam ser excitadas por um mecanismo qualquer. De acordo com a Termodinâmica, um mecanismo de excitação deve fornecer um aumento na entropia durante a fase de alta-temperatura do ciclo de oscilação. A dependência positiva da temperatura 
com a opacidade e a alta sensibilidade à temperatura da energia nuclear são conhecidas por serem os mecanismo de excitação chamados de mecanismo $\kappa$ e mecanismo $\varepsilon$, respectivamente (Unno et al., 1989). Existem também outros mecanismos de excitação. Para que um modo seja excitado, algum desses mecanismos deve estar operante na região onde a amplitude das autofunções seja grande. Descobriu-se que para as anãs brancas, os modos g são produzidos pelo mecanismo $\kappa$ de opacidade (e.g. Kepler et al., 1995).

\subsection{Estrelas Pulsantes na Sequência Principal}

Estrelas variáveis foram observadas em todo o diagrama HR (Unno et al., 1989). A Figura 1.1 mostra as diferentes classes de estrelas com oscilações no diagrama HR. Ao longo da sequência principal existem desde estrelas de baixa massa do tipo Solar até estrelas massivas ( $\beta$ Cephei). Essas estrelas geralmente apresentam espectros com muitos períodos e são alvos potencialmente mais interessantes para se investigar os interiores estelares via análise sismológica (Christensen-Dalsgaard, 2003).

As estrelas $\delta$ Scuti encontram-se no cruzamento da sequência principal e da faixa de instabilidade das Cefeidas. Elas possuem massa entre 1,5 e 2,5 $M_{\odot}$ e exibem modos p radiais e não-radiais e modos g (ver Rodríguez e Breger, 2001, para uma revisão). As $\delta$ Scuti são as estrelas pulsantes da sequência principal nas quais o maior número de modos de oscilação são observados (Cunha et al., 2007). A Figura 1.2 mostra o espectro de frequências para a estrela $\delta$ Scuti FG Virginis.

As $\beta$ Cephei são estrelas mais massivas, análogas às $\delta$ Scuti na sequência principal. As massas variam de 8 a $18 M_{\odot}$ e também exibem modos p radiais e não-radiais e modos g (ver Stankov e Handler, 2005, para uma revisão). A Figura 1.3 mostra o espectro da estrela $\beta$ Cephei HD129929.

No cruzamento da sequência principal com a faixa de instabilidade das Cefeidas encontramse também as estrelas roAp. Mesmo estando mais ou menos na mesma região de estrelas "normais "da sequência principal, essas estrelas apresentam contudo atmosferas peculiares, particularmente em relação à composição química e possuem ainda um forte campo magnético. Elas também giram mais lentamente que as estrelas $\delta$ Scuti, com pulsações que diferem bastante. Elas podem ser monoperiódicas ou multiperíodicas e são resultados de modos acústicos de alta frequência (Cunha et al., 2007). 


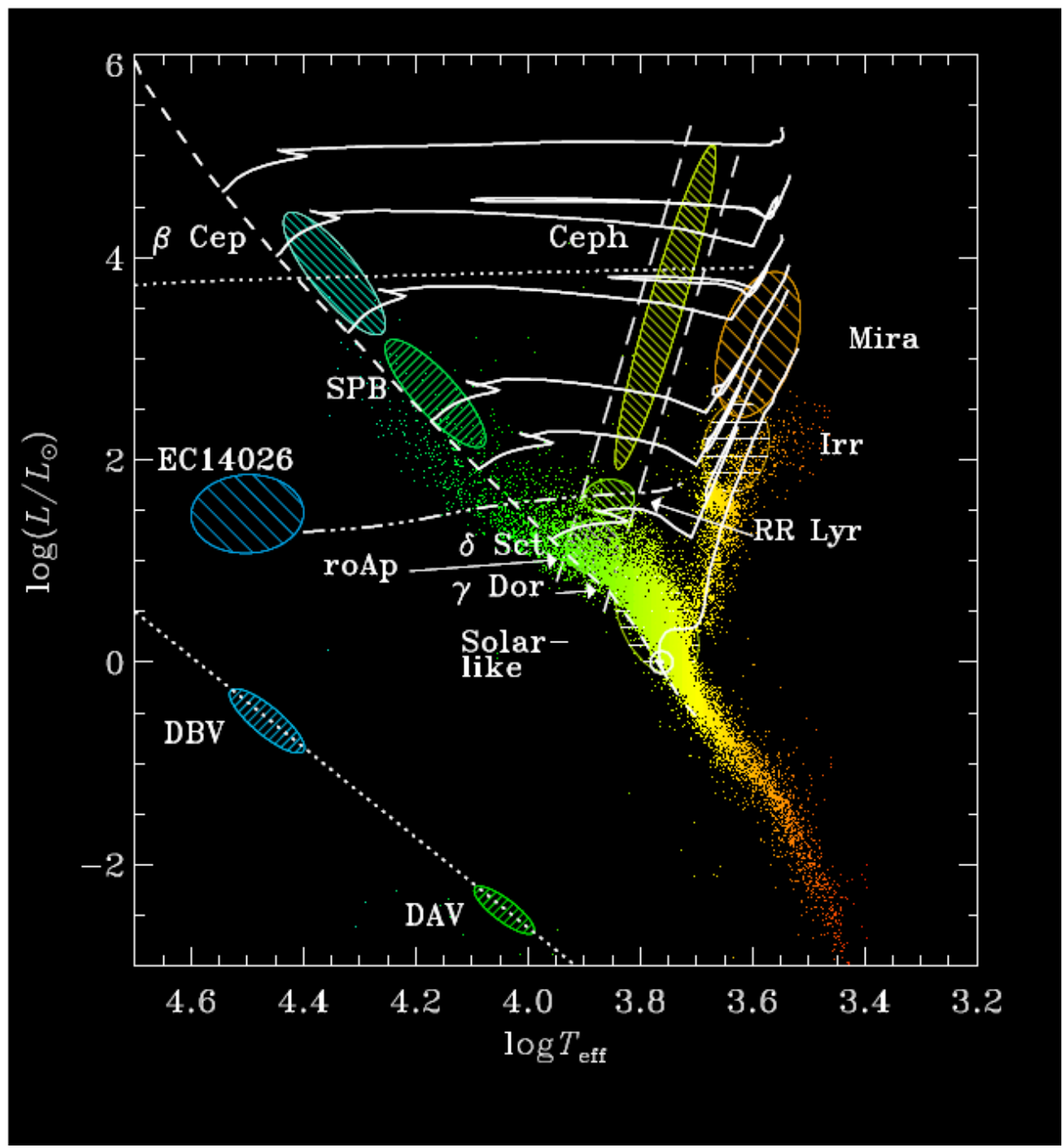

Figura 1.1: Diagrama HR representando as diferentes classes de estrelas pulsantes. Créditos: Favata et al. (2000).

Outra classe existente na sequência principal são as $\gamma$ Doradus e as estrelas B pulsante lentas (SPB - slowly pulsating B). Essas duas classes consistem de objetos com uma variedade de modos g, com períodos da ordem de 0,5 e 3 dias (Cunha et al., 2007).

Finalmente, abaixo das $\gamma$ Dor na sequência principal encontra-se as estrelas pulsantes do tipo solar. A Figura 1.4 mostra o espectro de frequências de 5 estrelas do tipo solar, incluindo o Sol. O espectro dessas estrelas é mais denso do que o das outras da sequência principal. Além do mais, as amplitudes são bem menores e elas mostram um formato "envelope "que é típico de oscilações excitadas estocasticamente (Cunha et al., 2007). 


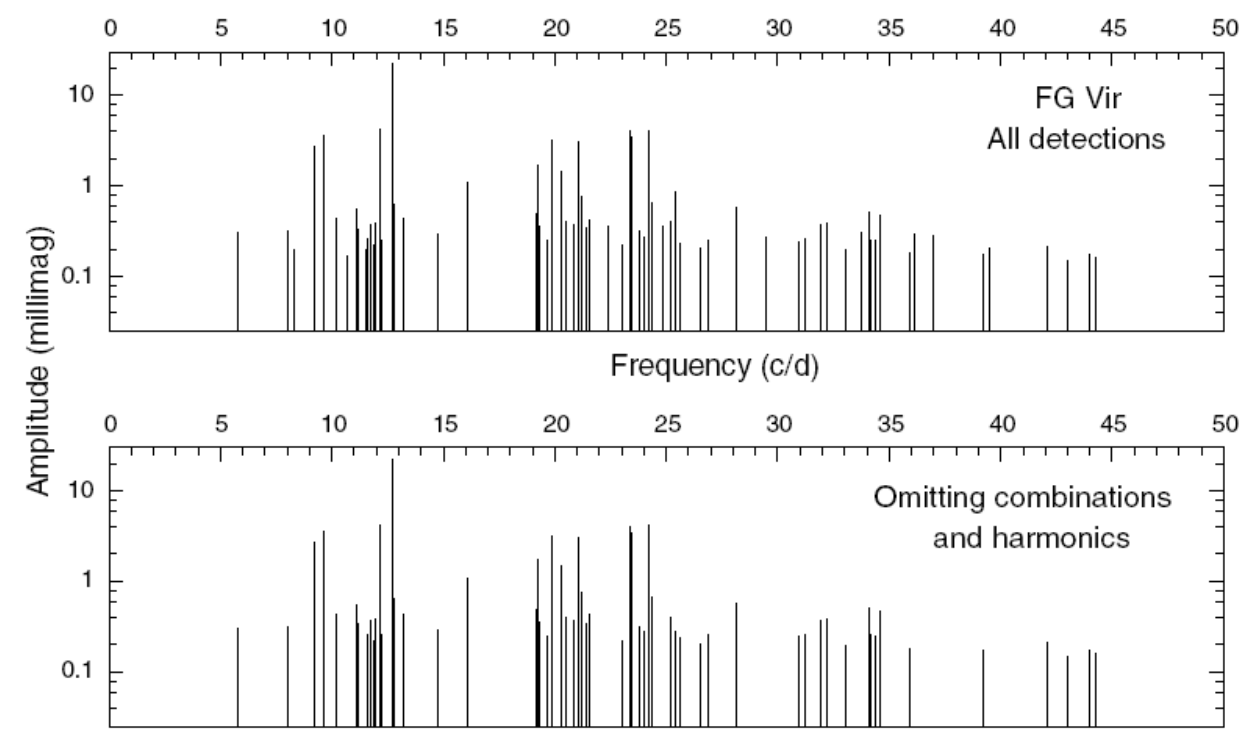

Figura 1.2: Espectro de frequências detectadas para a estrela $\delta$ Scuti FG Virginis. Créditos: Breger et al. (2005). 


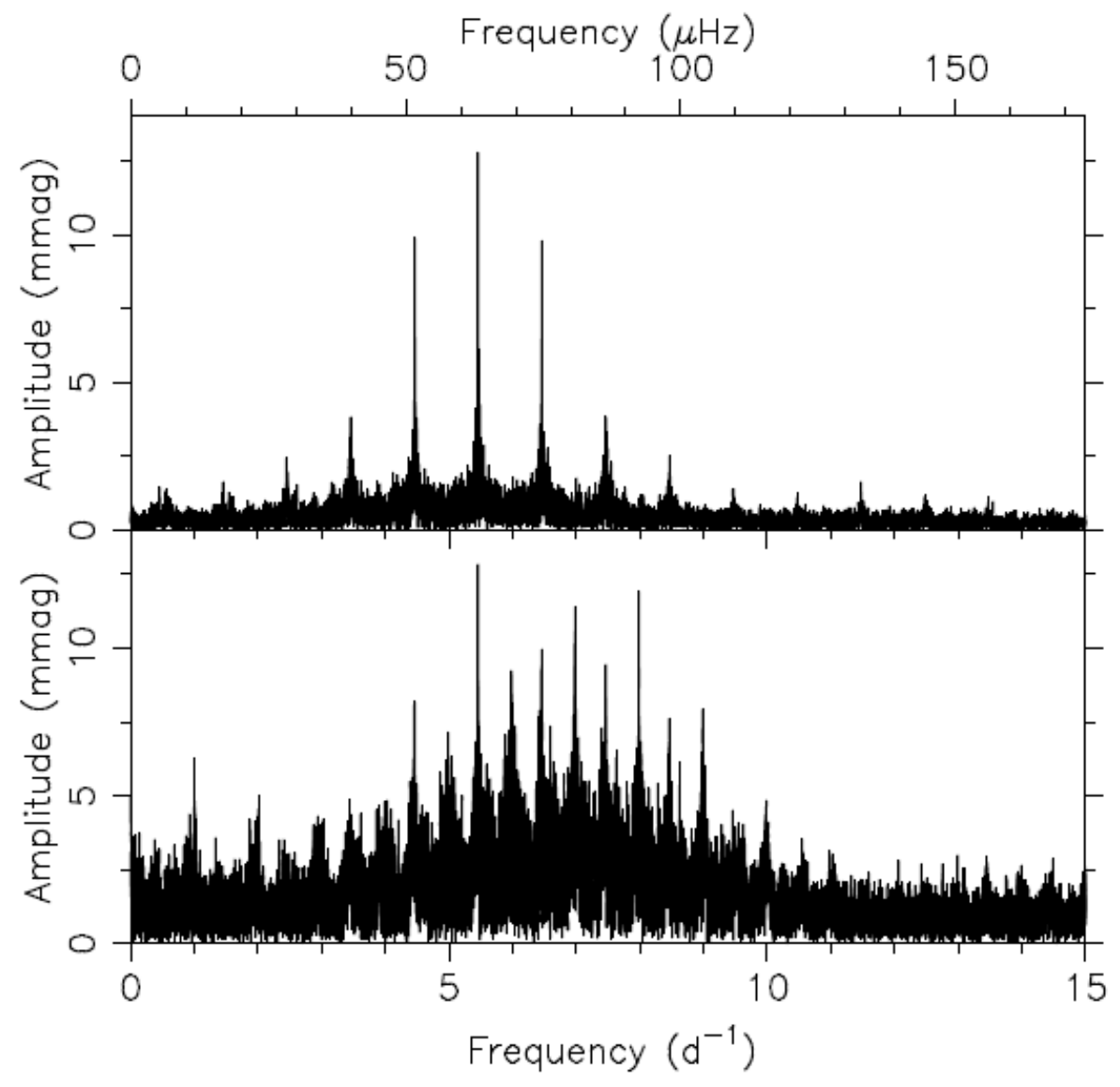

Figura 1.3: Espectro de frequências da estrela $\beta$ Cephei HD129929. O painel superior mostra a janela espectral gerada a partir da amplitude do modo dominante e dos dados de amostragem. Créditos: Cunha et al. (2007). 


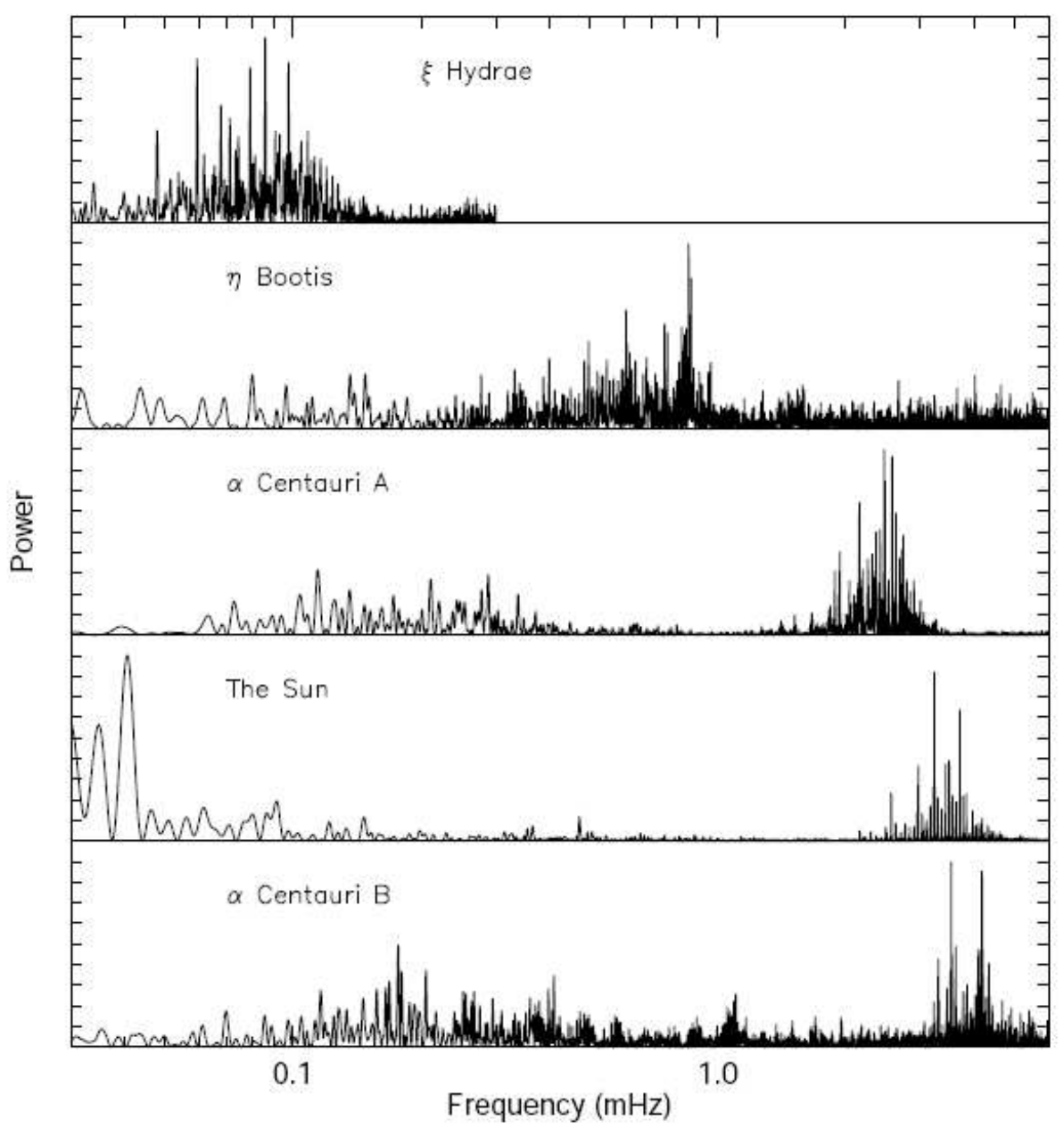

Figura 1.4: Espectro de frequências de estrelas do tipo solar, incluindo o Sol. A ordenada é arbitrária. Créditos: Cunha et al. (2007). 
Capítulo 2

\section{Pulsações Estelares}

Neste capítulo, a teoria das pulsações não-radiais e radiais é apresentado. Primeiramente, as equações básicas de hidrodinâmica são introduzidas na seção 2.1. Depois, na seção 2.2, as equações de oscilação são derivadas a partir da imposição de pequenas perturbações no equilíbrio da estrela. Já na seção 2.3, é feita a separação em variáveis radiais e horizontais do sistema de equações de oscilação. Logo após, na seção 2.4, as equações para oscilações lineares e adiabáticas são discutidas. As propriedades dos modos de oscilação são apresentadas na seção 2.5. Em seguida, na seção 2.6, é introduzida a análise assimptótica e depois na seção 2.7, é discutido a influência da introdução da rotação estelar nas oscilações. Por último, na seção 2.8, são apresentados os mecanismos de excitação dos modos de oscilação.

\subsection{Equações Básicas}

As equações básicas que descrevem as deformações e oscilações de uma estrela são as equações da hidrodinâmica (Unno et al., 1989). Considera-se um gás contínuo, de modo que suas propriedades possam ser especificadas em função de posição e tempo. Assim, levando em conta que a massa é conservada, a equação de continuidade pode ser expressa como

$$
\frac{d \rho}{d t}+\rho \vec{\nabla} \cdot \vec{v}=0
$$

onde $\rho$ é a densidade do gás e $\vec{v}$ é a velocidade deste.

No caso de oscilações estelares, pode-se ignorar normalmente o atrito interno do gás. Desse modo, considerando as forças agindo num volume de gás, a equação para conservação 
de momento é dada por

$$
\rho \frac{d \vec{v}}{d t}+\rho \vec{v} \cdot \vec{\nabla} \vec{v}=-\vec{\nabla} p-\rho \vec{\nabla} \Phi
$$

onde $p$ é a pressão e $\Phi$ representa o potencial gravitacional, que satisfaz a equação de Poisson

$$
\nabla^{2} \Phi=4 \pi G \rho
$$

cuja forma integral é

$$
\Phi(\vec{r}, t)=-G \int_{V} \frac{\rho\left(\vec{r}^{\prime}, t\right) d V}{\left|\vec{r}-\vec{r}^{\prime}\right|} .
$$

Para a decrição da estrutura estelar ficar completa, ainda é necessário uma relação entre a pressão e a densidade do gás. Utilizando a primeira lei da Termodinâmica e algumas identidades termodinâmicas, a equação para a conservação da energia pode ser expressa na forma

$$
\rho \frac{d q}{d t}=\rho \epsilon-\vec{\nabla} \cdot \vec{F}
$$

onde $\epsilon$ é a taxa de geração de energia por unidade de massa e $\vec{F}$ é o fluxo de energia. Para o caso de fluxo radiativo, este pode ser derivado da equação de difusão radiativa

$$
\overrightarrow{F_{R}}=-K \vec{\nabla} T
$$

onde a condutividade radiativa $K$ é escrita em termos da opacidade $\kappa$ como

$$
K=\frac{4 a c_{*}}{3 \kappa \rho} T^{3}
$$

para $a$ sendo a constante de densidade de radiação e $c_{*}$ a velocidade da luz.

Considerando agora a estrutura em estado de equilíbrio estático, esta obedece a equação de equilibrio hidrostático

$$
\vec{\nabla} p_{0}=\rho_{0} \overrightarrow{g_{0}}=-\rho_{0} \vec{\nabla} \Phi_{0}
$$

onde

$$
\overrightarrow{g_{0}}=-g_{0} \overrightarrow{a_{r}}
$$

Para o caso de simetria esférica, a equação 2.8 é reescrita em coordenadas polares esféricas $(r, \theta, \phi)$ ficando da seguinte forma

$$
\frac{d p_{0}}{d r}=-g_{0} \rho_{0}
$$


Da mesma maneira, a equação de Poisson pode ser integrada para gerar

$$
g_{0}=\frac{G}{r^{2}} \int_{0}^{r} 4 \pi \rho_{0} r^{\prime 2} d r^{\prime}=\frac{G m_{0}(r)}{r^{2}},
$$

onde $m_{0}(r)$ é a massa no interior da esfera de raio $r$. O fluxo é irradiado radialmente

$$
\vec{F}=F_{r, 0} \overrightarrow{a_{r}},
$$

de modo que a equação de conservação de energia é dada por

$$
\rho_{0} \epsilon_{0}=\frac{1}{r^{2}} \frac{d}{d r}\left(r^{2} F_{r, 0}\right)=\frac{1}{4 \pi r^{2}} \frac{d L_{0}}{d r},
$$

onde $L_{0}=4 \pi r^{2} \rho \epsilon$, e consequentemente,

$$
\frac{d L_{0}}{d r}=4 \pi r^{2} \rho_{0} \epsilon_{0}
$$

Finalmente a expressão de difusão do fluxo é

$$
\frac{d T_{0}}{d r}=-\frac{3 \kappa_{0} \rho_{0}}{16 \pi a r^{2} c_{*} T_{0}^{3}},
$$

onde $\kappa_{0}$ é a opacidade, a é a constante de densidade de radiação e $c_{*}$ é a velocidade da luz. A equação 2.15 só é válida quando a energia é transportada integralmente por processos radiativos. No caso de existirem processos convectivos, o fluxo convectivo deve ser incluido de modo que o fluxo total de energia corresponde a soma dos dois fluxos,

$$
F=F_{R}+F_{C}
$$

e

$$
L_{r}=4 \pi r^{2}\left(F_{R}+F_{C}\right) \text {, }
$$

onde $F_{R}$ e $F_{C}$ são os fluxos radiativo e convectivo, respectivamente.

\subsection{Equações de Oscilação}

Para derivar as equações básicas da oscilação linear, considera-se uma estrutura em estado de equilíbrio onde são impostas pequenas perturbações. Assume-se na teoria linear que as perturbações são suficientemente pequenas de modo que somente os termos de primeira ordem são importantes e os termos de ordem superior podem ser desprezadas. 
Existem duas maneiras de expressar a perturbação: a forma Euleriana e a forma Lagrangeana. A perturbação euleriana é definida como a perturbação de uma quantidade física numa certa posição, enquanto que a perturbação Lagrangeana é definida como a perturbação num dado elemento de fluído. Assim, como exemplo, a pressão pode ser expressa por

$$
p(\vec{r}, t)=p_{0}(\vec{r})+p^{\prime}(\vec{r}, t)
$$

para a notação Euleriana, ou,

$$
p(\vec{r}, t)=p_{0}\left(\overrightarrow{r_{0}}\right)+\delta p\left(\overrightarrow{r_{0}}, t\right)
$$

para a Lagrangeana. As duas estão relacionadas por

$$
\delta p(\vec{r}, t)=p^{\prime}\left(\overrightarrow{r_{0}}, t\right)+\vec{\xi} \cdot \vec{\nabla} p_{0}(\vec{r})
$$

onde $\vec{\xi}$ é o vetor deslocamento

$$
\vec{\xi} \equiv \vec{r}-\overrightarrow{r_{0}}
$$

que mostra o deslocamento do elemento de fluido da posição de equilíbrio $r_{0}$.

Aplicando essas perturbações nas equações básicas da hidrodinâmica 2.1, 2.2, 2.3, 2.4 e 2.8, a equação da continuidade fica

$$
\frac{\partial \rho^{\prime}}{\partial t}+\nabla \cdot\left(\rho_{0} v\right)=0
$$

ou usando a velocidade com que essa perturbação se propaga e integrando no tempo,

$$
\rho^{\prime}+\nabla \cdot\left(\rho_{0} \delta r\right)=0
$$

A equação de movimento perturbada é

$$
\rho_{0} \frac{\partial^{2} \delta r}{\partial t^{2}}=\rho_{0} \frac{\partial v}{\partial t}=-\nabla p^{\prime}+\rho_{0} g^{\prime}+\rho^{\prime} g_{0}
$$

onde a perturbação no potencial gravitacional também deve satisfazer a equação de Poisson pertubada

$$
\nabla^{2} \Phi^{\prime}=4 \pi G \rho^{\prime}
$$

com solução equivalente a equação 2.4 ,

$$
\Phi^{\prime}=-G \int_{V} \frac{\rho^{\prime}\left(r^{\prime}, t\right)}{\left|r-r^{\prime}\right|} d V .
$$


Para a equação da energia,

$$
\frac{d p}{d t}=\frac{\partial p}{\partial t}+\vec{v} \cdot \vec{\nabla} p=\frac{\partial p^{\prime}}{\partial t}+\vec{v} \cdot \vec{\nabla} p_{0}=\frac{\partial p^{\prime}}{\partial t}+\frac{\partial \overrightarrow{\delta r}}{\partial t} \cdot \vec{\nabla} p_{0}=\frac{\partial}{\partial t}(\delta p),
$$

resultando na pertubação para a taxa de calor

$$
\rho_{0} \frac{\partial \delta q}{\partial t}=\delta(\rho \epsilon-\vec{\nabla} \cdot \vec{F})=(\rho \epsilon)^{\prime}-\vec{\nabla} \cdot \vec{F}^{\prime}
$$

onde

$$
\vec{F}^{\prime}=-K_{0} \vec{\nabla} T^{\prime}-K^{\prime} \vec{\nabla} T_{0} .
$$

As equações 2.22, 2.24, 2.25, 2.28 e 2.29 são equações diferenciais parciais lineares e homogêneas com respeito ao tempo $t$ e ao espaço de coordenadas $\vec{r}$ para as variáveis perturbadas. Essas equações contêm também quantidades de equilíbrio, denotadas com sobrescrito, que dependem somente da coordenada radial. Dessa forma, é possível separar a variável tempo e escrever as grandezas como proporcionais a exp $(i \omega t)$ (Unno et al., 1989).

\subsection{Equações de Onda nas Direções Radial e Angular}

O sistema de equações diferenciais encontrado na seção 2.2 pode ser separado ainda em coordenadas horizontais e radiais de modo a facilitar a solução. Para o deslocamento isso leva a

$$
\delta r=\xi_{r} a_{r}+\xi_{h}
$$

A componente horizontal na equação do movimento 2.24 satisfaz

$$
\rho_{0} \frac{\partial^{2} \xi_{h}}{\partial t^{2}}=-\nabla_{h} p^{\prime}-\rho_{0} \nabla_{h} \Phi^{\prime}
$$

mas como o gradiente horizontal das quantidades de equilíbrio é zero, a equação fica

$$
\rho_{0} \frac{\partial^{2}}{\partial t^{2}} \nabla_{h} \cdot \xi_{h}=-\nabla_{h}^{2} p^{\prime}-\rho_{0} \nabla_{h}^{2} \Phi^{\prime}
$$

A equação da continuidade 2.23 pode ser escrita como

$$
\rho^{\prime}=\frac{1}{r^{2}} \frac{\partial}{\partial r}\left(\rho_{0} r^{2} \xi_{r}\right)-\rho_{0} \nabla \_h^{2} \cdot \Phi^{\prime} .
$$

A equação 2.33 pode ser combinada com a equação 2.32, obtendo-se

$$
-\frac{\partial^{2}}{\partial t^{2}}\left[\rho^{\prime}+\frac{1}{r^{2}} \frac{\partial}{\partial r}\left(r^{2} \rho_{0} \xi_{r}\right)\right]=-\nabla_{h}^{2} p^{\prime}-\rho_{0} \nabla_{h}^{2} \Phi^{\prime} .
$$


A componente radial do movimento 2.24 se torna

$$
\rho_{0} \frac{\partial^{2} \xi_{r}}{\partial t^{2}}=-\frac{\partial p^{\prime}}{\partial r}-\rho^{\prime} g_{0}-\rho_{0} \frac{\partial \Phi^{\prime}}{\partial r} .
$$

E finalmente, a equação de Poisson fica

$$
\frac{1}{r^{2}} \frac{\partial}{\partial r}\left(r^{2} \frac{\partial \Phi^{\prime}}{\partial r}\right)+\nabla_{h}^{2} \Phi^{\prime}=4 \pi G \rho^{\prime} .
$$

Agora, separa-se as variáveis angulares, para um $f$ igual a uma autofunção do operador Laplace horizontal

$$
\nabla_{h}^{2} f=-\frac{1}{r^{2}} \Lambda f
$$

onde $\Lambda$ é uma constante, ou de forma extensa,

$$
\frac{1}{\sin \theta} \frac{\partial}{\partial \theta}\left(\sin \theta \frac{\partial f}{\partial \theta}\right)+\frac{1}{\sin ^{2} \theta} \frac{\partial^{2} f}{\partial \phi^{2}}=-\Lambda f .
$$

Os coeficientes são indepentes de $\phi$ e $f$ pode ser escrita separando a dependência nas variáveis angulares

$$
f(\theta, \phi)=f_{1}(\theta) f_{2}(\phi) .
$$

A equação para $f_{2}$ é

$$
\frac{d^{2} f_{2}}{d \phi^{2}}=\alpha f_{2}
$$

onde $\alpha$ é outra constante; a equação tem solução $f_{2}=\exp \left( \pm \alpha^{1 / 2} \phi\right)$. No entanto, a solução tem que ser continua para $f_{2}(0)=f_{2}(2 \pi)$ e consequentemente $\alpha^{1 / 2}=i m$, onde $m$ é inteiro.

Quando essas condições são usadas na equação 2.38, resulta na seguinte equação diferencial para $f_{1}$ :

$$
\frac{d}{d x}\left[\left(1-x^{2}\right) \frac{d f_{1}}{d x}\right]+\left(\Lambda-\frac{m^{2}}{1-x^{2}}\right) f_{1}=0
$$

onde $x=\cos \theta$. Através das condições de contorno, essa equação tem solução regular somente quando

$$
\Lambda=l(l+1),
$$

onde $l$ é um número inteiro não negativo e

$$
|m| \leq l
$$

Então, a solução regular é

$$
f_{1}(\theta)=P_{l}^{m}(\cos \theta)
$$


onde $P_{l}^{m}$ é o polinômio de Legendre para $l$ e $m$. Desse modo, a função $f$ pode ser escrita como

$$
f(\theta, \phi)=(-1)^{m} c_{l m} P_{l}^{m}(\cos \theta) \exp (i m \phi) \equiv Y_{l}^{m}(\theta, \phi),
$$

onde $Y_{l}^{m}$ são harmônicos esféricos e $c_{l m}$ é a constante de normalização de modo que a integral do quadrado do módulo destes sobre uma esfera seja unitária. A solução obedece a seguinte equação de autovalores

$$
\nabla_{h}^{2} f=-\frac{l(l+1)}{r^{2}} f
$$

As variáveis independentes nas equações 2.34 - 2.36 podem ser escritas agora como

$$
\xi_{r}(r, \theta, \phi, t)=\sqrt{4 \pi} \tilde{\xi}_{r}(r) Y_{l}^{m}(\theta, \phi) \exp (-i \omega t)
$$

e

$$
p^{\prime}(r, \theta, \phi, t)=\sqrt{4 \pi} \tilde{p}^{\prime}(r) Y_{l}^{m}(\theta, \phi) \exp (-i \omega t)
$$

Introduzindo-se as equações de perturbação segue que

$$
\begin{gathered}
\omega^{2}\left[\tilde{\rho}^{\prime}+\frac{1}{r^{2}} \frac{d}{d r}\left(r^{2} \rho_{0} \tilde{\xi}_{r}\right)\right]=\frac{l(l+1)}{r^{2}}\left(\tilde{p}^{\prime}+\rho_{0} \tilde{\Phi}^{\prime}\right) \\
-\omega^{2} \rho_{0} \tilde{\xi}_{r}=-\frac{d \tilde{p}^{\prime}}{d r}-\tilde{\rho}^{\prime} g_{0}-\rho_{0} \frac{d \tilde{\Phi}^{\prime}}{d r} \\
\frac{1}{r^{2}} \frac{d}{d r}\left(r^{2} \frac{d \tilde{\Phi}^{\prime}}{d r}\right)-\frac{l(l+1)}{r^{2}} \tilde{\Phi}^{\prime}=4 \pi G \tilde{\rho}
\end{gathered}
$$

juntamente com

$$
\left(\delta \tilde{p}-\frac{\Gamma_{1,0} p_{0}}{\rho_{0}} \delta \tilde{\rho}\right)=\rho_{0}\left(\Gamma_{3,0}-1\right) \delta \tilde{q}
$$

As equações 2.49 - 2.52 formam um sistema de equações para oscilações estelares lineares e não dependem de $m$ devido que foi assumida simetria esférica. Qualquer força que quebre essa simetria, fará a introdução da dependência com $m$ nos modos de oscilação.

Para oscilações radiais (caso epecífico para $l=0$ ), a pertubação no campo gravitacional pode ser eliminada analiticamente. Da equação 2.49, usando a equação 2.33 com a parte horizontal igual a zero,

$$
\frac{1}{r^{2}} \frac{d}{d r}\left(r^{2} \frac{d \tilde{\Phi}^{\prime}}{d r}\right)=-\frac{4 \pi G}{r^{2}} \frac{d}{d r}\left(r^{2} \rho_{0} \tilde{\xi}_{r}\right)
$$


ou, como a força gravitacional deve ser finita em $r=0$,

$$
\frac{d \tilde{\Phi}^{\prime}}{d r}=-4 \pi G \rho_{0} \tilde{\xi}_{r}
$$

Com essas eliminações, as equações de oscilação podem ser reduzidas a uma forma relativamente simples. A equação 2.52 se torna

$$
\tilde{\rho}^{\prime}=\frac{\rho_{0}}{\Gamma_{1,0} p_{0}} \tilde{p}^{\prime}+\rho_{0} \tilde{\xi}_{r}\left(\frac{1}{\Gamma_{1,0} p_{0}} \frac{d p_{0}}{d r}-\frac{1}{\rho_{0}} \frac{d \rho_{0}}{d r}\right)-\frac{\rho_{0}^{2}}{\Gamma_{1,0} p_{0}}\left(\Gamma_{3,0}-1\right) \delta \tilde{q} .
$$

E a equação 2.49 pode ser escrita como

$$
\frac{d \tilde{\xi}_{r}}{d r}=-\frac{2}{r} \tilde{\xi}_{r}-\frac{1}{\Gamma_{1,0} p_{0}} \frac{d p_{0}}{d r} \tilde{\xi}_{r}-\frac{\rho_{0}}{\Gamma_{1,0} p_{0}} \tilde{p}^{\prime}+\frac{\rho_{0}}{\Gamma_{1,0} p_{0}}\left(\Gamma_{3,0}-1\right) \delta \tilde{q},
$$

ou, introduzindo $\zeta \equiv \tilde{\xi}_{r} / r$,

$$
\tilde{p}^{\prime}=-\Gamma_{1,0} p_{0} r\left(\frac{d \zeta}{d r}+\frac{3}{r} \zeta+\frac{1}{\Gamma_{1,0} p_{0}} \frac{d p_{0}}{d r} \zeta\right)+\rho_{0}\left(\Gamma_{3,0}-1\right) \delta \tilde{q}
$$

Substituindo as equações 2.54, 2.55 e 2.57 em 2.50, obtém-se, depois de muita manipulação,

$$
\frac{1}{r^{3}} \frac{d}{d r}\left(r^{4} \Gamma_{1,0} p_{0} \frac{d \zeta}{d r}\right)+\frac{d}{d r}\left[\left(3 \Gamma_{1,0}-4\right) p_{0}\right] \zeta+\rho_{0} \omega^{2} r \zeta=\frac{d}{d r}\left[\rho_{0}\left(\Gamma_{3,0}-1\right) \delta \tilde{q}\right] .
$$

É importante notar que a equação 2.58 esconde um grande grau de complexidade no termo de calor do lado direito. Apesar disso, essa equação é conveniente para discutir as propriedades das oscilações radiais (Christensen-Dalsgaard, 2003).

\subsection{Oscilações Adiabáticas}

As propriedades dinâmicas podem ser estudadas assumindo que $\delta q=0$, ou seja, que ocorrem adiabaticamente. Dessa forma, a equação 2.52 pode ser escrita como

$$
\rho^{\prime}=\frac{\rho}{\Gamma_{1} p} p^{\prime}+\rho \xi_{r}\left(\frac{1}{\Gamma_{1} p} \frac{d p}{d r}-\frac{1}{\rho} \frac{d \rho}{d r}\right) .
$$

Essa equação pode ser usada para eliminar $\rho^{\prime}$ das equações 2.49 - 2.51. Da equação 2.49 obtém-se

$$
\frac{d \xi_{r}}{d r}=-\left(\frac{2}{r}+\frac{1}{\Gamma_{1} p} \frac{d p}{d r}\right) \xi_{r}+\frac{1}{\rho}\left[\frac{l(l+1)}{\omega^{2} r^{2}}-\frac{1}{c^{2}}\right] p^{\prime}+\frac{l(l+1)}{\omega^{2} r^{2}} \Phi^{\prime},
$$

onde $c^{2}=\Gamma_{1} p / \rho$ é o quadrado da velocidade do som adiabática.

É conveniente introduzir a frequência acústica característica $S_{l}$ definida por

$$
S_{l}^{2}=\frac{l(l+1) c^{2}}{r^{2}}=k_{h}^{2} c^{2} .
$$


Assim a equação 2.60 pode ser escrita da seguinte forma

$$
\frac{d \xi_{r}}{d r}=-\left(\frac{2}{r}+\frac{1}{\Gamma_{1} p} \frac{d p}{d r}\right) \xi_{r}+\frac{1}{\rho c^{2}}\left(\frac{S_{l}^{2}}{\omega^{2}}-1\right) p^{\prime}+\frac{l(l+1)}{\omega^{2} r^{2}} \Phi^{\prime}
$$

Definida a frequência de empuxo (Brunt-Väisälä frequency), frequência com que uma bolha de gás podem oscilar verticalmente em torno da sua posição de equilíbrio sobre efeito da gravidade, da forma

$$
N^{2}=g\left(\frac{1}{\Gamma_{1} p} \frac{d p}{d r}-\frac{1}{\rho} \frac{d \rho}{d r}\right)
$$

a equação 2.50 fica

$$
\frac{d p^{\prime}}{d r}=\rho\left(\omega^{2}-N^{2}\right) \xi_{r}+\frac{1}{\Gamma_{1} p} \frac{d p}{d r} p^{\prime}-\rho \frac{d \Phi^{\prime}}{d r} .
$$

Finalmente, a equação 2.51 fica

$$
\frac{1}{r^{2}} \frac{d}{d r}\left(r^{2} \frac{d \Phi^{\prime}}{d r}\right)=4 \pi G\left(\frac{p^{\prime}}{c^{2}}+\frac{\rho \xi_{r}}{g} N^{2}\right)+\frac{l(l+1)}{r^{2}} \Phi^{\prime} .
$$

As equações 2.62, 2.64 e 2.65 constituem um sistema de quarta ordem de equações diferenciais ordinárias com quatro variáveis independentes $\xi_{r}, p^{\prime}, \Phi^{\prime}$ e $d \Phi^{\prime} / d r$. Para resolver esse sistema é necessário definir as condições de contorno. Considera-se as condições no centro $(r=0)$ e na superfície $(p=0)$ da estrela.

Próximo ao centro, $\xi_{r}$ se comporta como $r^{l-1}$, enquanto que $p^{\prime}$ e $\Phi^{\prime}$ se comportam como $r^{l}$. No entanto, para o caso específico de oscilações radiais $(l=0), \xi_{r}$ se comporta como r. No caso particular de $l>0$

$$
\xi_{r} \simeq l \xi_{h}, \quad \text { para } r \rightarrow 0
$$

Uma condição de contorno da superfície é obtida exigindo-se a continuidade de $\Phi$ e de sua derivada em $r=R$, onde $R$ é igual ao raio da superfície da estrela. Fora da estrela, a perturbação de densidade é zero, e a equação de Poisson pode ser resolvida analiticamente. Para a solução igual a zero no infinito

$$
\Phi^{\prime}=A r^{-l-1}
$$

onde $A$ é uma constante. Consequentemente $\Phi^{\prime}$ tem que satisfazer

$$
\frac{d \Phi^{\prime}}{d r}+\frac{l+1}{r} \Phi^{\prime}=0, \quad \text { em } r=R
$$

A outra condição depende do tratamento para modelar a atmosfera estelar. Note que para uma fronteira bem definida em $r=R$, é razoável considerar que nenhuma força atua 
nela e a pressão deve permanecer constante, assim, a segunda condição de contorno leva a

$$
\delta p=p^{\prime}+\xi_{r} \frac{d p}{d r}=0, \quad \text { em } r=R
$$

Uma análise mais detalhada do comportamento das oscilações na atmosfera leva a um resultado semelhante, exceto para altas frequências (Christensen-Dalsgaard, 2003).

Da equação 2.69, pode-se estimar a razão entre as componentes de deslocamento radial e horizontal das ondas. Na maioria dos casos a perturbação gravitacional é pequena e desse modo, pode-se obter

$$
\frac{\xi_{h}(R)}{\xi_{r}(R)}=\frac{g_{s}}{R \omega^{2}} \equiv \sigma^{-2}
$$

onde $g_{s}$ é a gravidade na superfície e $\sigma$ é a frequência adimensional definida por

$$
\sigma^{2}=\frac{R^{3}}{G M} \omega^{2}
$$

Assim, o valor da razão $\xi_{h} / \xi_{r}$ depende apenas da frequência nessa aproximação $O$ valor médio quadrático é

$$
\frac{\delta h_{r m s}}{\delta r_{r m s}}=\frac{\sqrt{l(l+1)}}{\sigma^{2}} \quad \text { em } r=R .
$$

Para o caso das oscilações de 5 minutos do Sol, $\sigma^{2} \sim 1000$ e o movimento é predominantemente vertical, exceto para 1 grande.

\subsection{Propriedades dos Modos de Oscilação}

Resolver o sistema de equações das oscilações não é trivial. Porém, algumas aproximações levam a soluções simples que podem ser utilizadas para estudar algumas propriedades interessantes dos modos de oscilação.

\subsubsection{Aproximação de Cowling}

Resolver o sistema de quarta ordem exige um estudo assimptótico elaborado. Felizmente a perturbação do potencial gravitacional pode ser frequentemente desprezada. Considerando a solução da integral em 2.26 da equação de Poisson

$$
\Phi^{\prime}(r)=-\frac{4 \pi G}{2 l+1}\left[\frac{1}{r^{l+1}} \int_{0}^{r} \rho^{\prime}\left(r^{\prime}\right) r^{\prime l+2} d r^{\prime}+r^{l} \int_{r}^{R} \frac{\rho^{\prime}\left(r^{\prime}\right)}{r^{\prime l-1}} d r^{\prime}\right] .
$$

Da equação 2.73 segue que $\Phi^{\prime}$ é pequeno em relação a $\rho^{\prime}$ para 
- 1 grande;

- $\mathbf{n}$ grande.

Assim, nestas condições, $\Phi^{\prime}$ pode ser desprezado, reduzindo o sistema para somente duas equações.

\subsubsection{Aprisionamento dos Modos}

As equações na aproximação de Cowling podem serem escritas como

$$
\begin{gathered}
\frac{d \xi_{r}}{d r}=-\left(\frac{2}{r}-\frac{1}{\Gamma_{1}} H_{p}^{-1}\right) \xi_{r}+\frac{1}{\rho c^{2}}\left(\frac{S_{l}^{2}}{\omega^{2}}-1\right) p^{\prime} \\
\frac{d p^{\prime}}{d r}=\rho\left(\omega^{2}-N^{2}\right) \xi_{r}-\frac{1}{\Gamma_{1}} H_{p}^{-1} p^{\prime}
\end{gathered}
$$

onde

$$
H_{p}^{-1}=-\frac{d \ln p}{d r}
$$

Para oscilações de alta ordem radial, as autofunções variam muito mais rapidamente que as quantidades de equilíbrio e dessa maneira, essas podem ser desprezadas, reduzindo as equações a

$$
\begin{aligned}
\frac{d \xi_{r}}{d r} & =\frac{1}{\rho c^{2}}\left(\frac{S_{l}^{2}}{\omega^{2}}-1\right) p^{\prime} \\
\frac{d p^{\prime}}{d r} & =\rho\left(\omega^{2}-N^{2}\right) \xi_{r} .
\end{aligned}
$$

Combinando 2.77 com 2.78 e desprezando mais uma vez as derivadas das quantidades de equilíbrio resulta em

$$
\frac{d^{2} \xi_{r}}{d r^{2}}=\frac{\omega^{2}}{c^{2}}\left(1-\frac{N^{2}}{\omega^{2}}\right)\left(\frac{S_{l}^{2}}{\omega^{2}}-1\right) \xi_{r},
$$

que pode ser escrita como

$$
\frac{d^{2} \xi_{r}}{d r^{2}}=-K(r) \xi_{r}
$$

onde

$$
K(r)=\frac{\omega^{2}}{c^{2}}\left(\frac{N^{2}}{\omega^{2}}-1\right)\left(\frac{S_{l}^{2}}{\omega^{2}}-1\right) .
$$

O comportamento local de $\xi_{r}$ depende do sinal de $K$. Um modo oscila como função de r para $K(r)>0$ e varia expoencialmente onde $K(r)<0$. Pontos onde $K(r)=0$ são 
pontos de mudança do modo. Na maioria dos casos, o modo tem amplitude considerável em apenas uma região de propagação. A solução dessa equação é aproximadamente

$$
\begin{aligned}
& \xi_{r} \sim \cos \left(\int K^{1 / 2} d r+\phi\right), \quad K>0, \\
& \xi_{r} \sim \exp \left( \pm \int|K|^{1 / 2} d r\right), \quad K<0 .
\end{aligned}
$$

Assim, as condições para a solução oscilar são

$$
\text { o1) }|\omega|>|N| \text { e }|\omega|>S_{l}
$$

$\mathrm{Ou}$

$$
\text { o2) }|\omega|<|N| \text { e }|\omega|<S_{l}
$$

e para ter comportamento exponencial são

$$
\text { e1) }|N|<|\omega|<S_{l} \text {, }
$$

$\mathrm{Ou}$

$$
\text { e2) } \quad S_{L}<|\omega|<|N| \text {. }
$$

Para um dado modo de oscilação, podem existir diversas regiões onde a solução oscila, de acordo com o critério o1) e $o 2$ ), com regiões de interferência onde esta é exponencial. Contudo, geralmente uma dessas regiões de oscilação é dominante, com a solução decrescendo exponencialmente fora dela. A solução, então, é considerada aprisionada nessa região. As fronteiras entre as regiões de aprisionamento são normalmente encontradas para $K(r)=0$; esses pontos são chamados de pontos de retorno.

A Figura 2.1 mostra o comportamento das frequências $S_{l}$ e $N$. Como o sinal de $K$ está relacionado com essas frequências, é possível observar que o comportamento destas leva a duas classes de modos:

- modos com frequências altas que satisfazem a condição o1), chamados modos p;

- modos com frequências baixas que satisfazem a condição o2), chamados modos g.

No caso de estrelas evoluídas, o valor de $N$ no núcleo pode atingir valores altos. Nesse caso, $K$ pode ser positivo em altas frequências tanto na parte mais exterior onde $\omega>N, S_{l}$, o modo se comportando como um modo $\mathrm{p}$, e no núcleo onde $\omega<S_{l}, N$, o modo se comportando como modo g, dando origem assim a modos mistos (Cunha et al., 2007). 


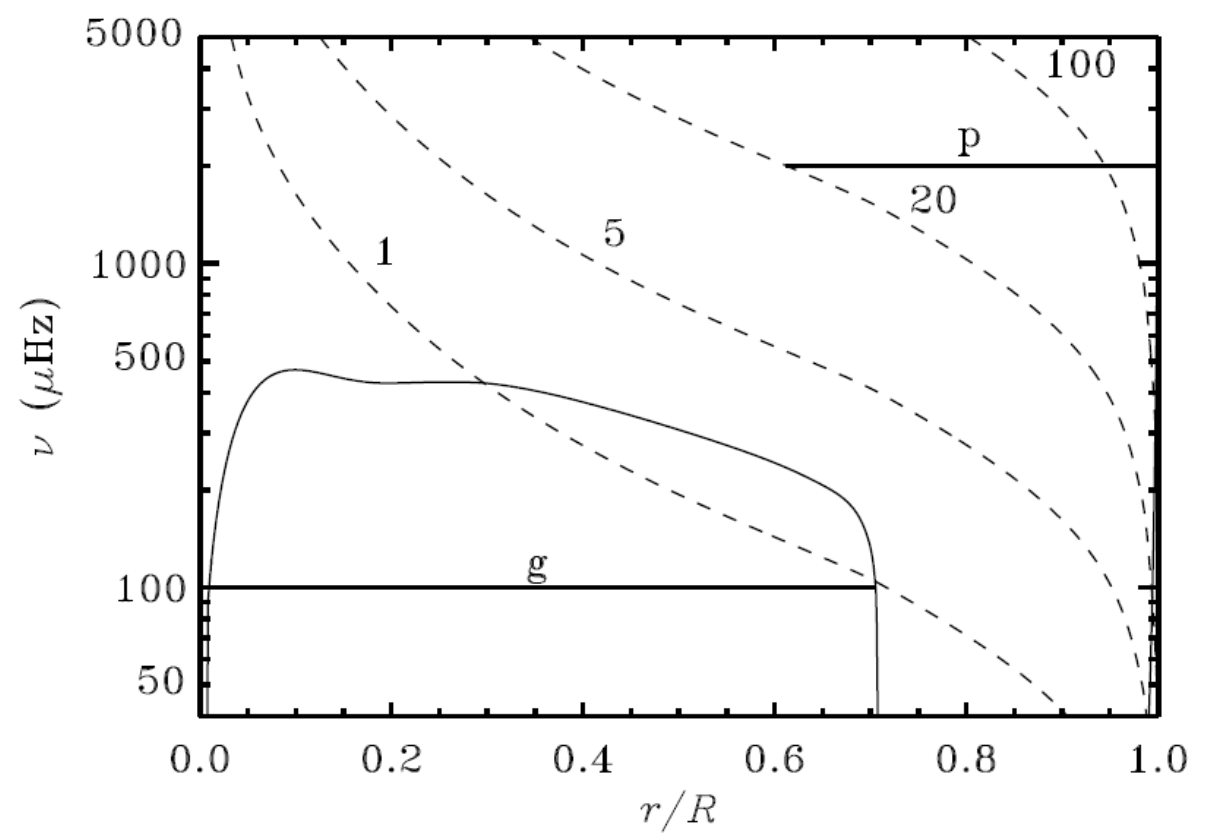

Figura 2.1: A linha contínua apresenta o comportamento da frequência de empuxo $N$ e linha tracejada, o da frequência acústica característica em termos da frequência cíclica correspondente, contra o raio fracional $r / R$, para um modelo Solar. As linha contínuas horizontais mostram as regiões de aprisionamento para um modo g com frequência $\nu=100 \mu H z$, e para um modo p com grau 20 e $\nu=2000 \mu H z$. Créditos: Christensen-Dalsgaard (2003).

\subsubsection{Modos $p$}

Nas regiões onde $\mathrm{N}$ é muito baixo, um modo de alta frequência é predominantemente controlado pelo comportamento de $S_{l}$; a autofunção oscila em função de r entre a superfície e o ponto de retorno inferior $r=r_{t}$ para o qual $S_{l}\left(r_{t}\right)=\omega$ ou

$$
\frac{c^{2}\left(r_{t}\right)}{r_{t}^{2}}=\frac{\omega^{2}}{l(l+1)}
$$

esta condição determina $r_{t}$ como função de $l$ e $\omega$. É óbvio que para graus baixos, $r_{t}$ é pequeno e o modo penetra em quase toda a estrela, incluindo partes do núcleo. Em particular, os modos p radial se extendem essencialmente até o centro da estrela. O comportamento desses modos pode ser visualizado na Figura 2.2a. É possível observar que os modos p são aprisionados entre a superfície e os pontos de retorno.

Para modos p, normalmente $\omega \gg N$ e então $K$ é aproximadamente

$$
K(r) \simeq \frac{1}{c^{2}}\left(\omega^{2}-S_{l}^{2}\right) .
$$




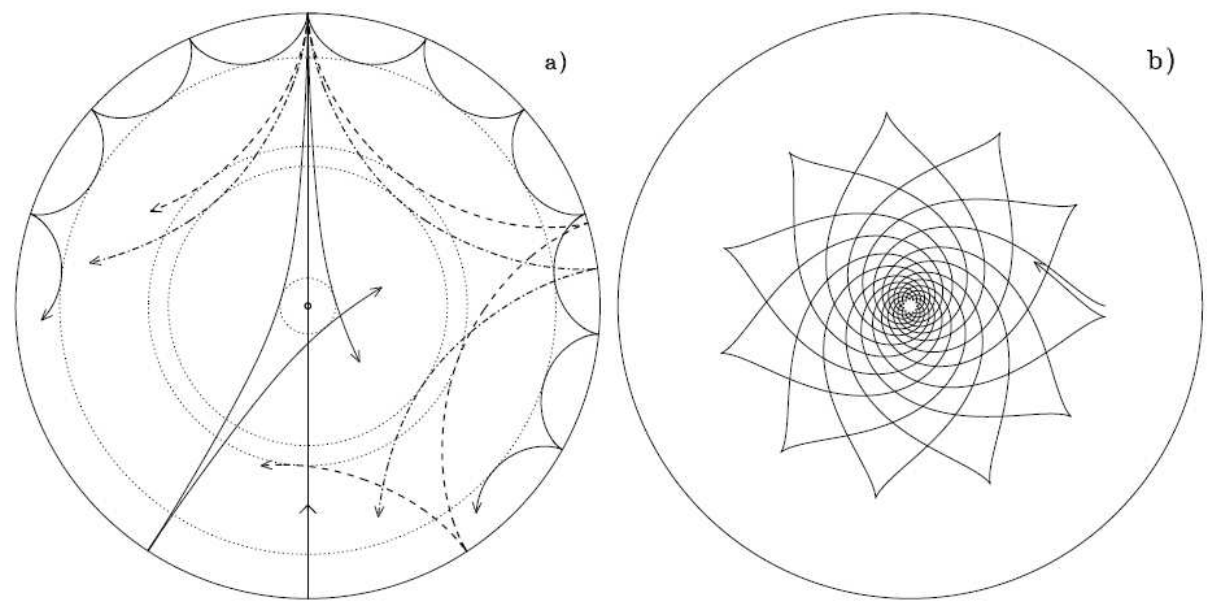

Figura 2.2: Propagação de ondas acústicas ou de gravidade na seção transversal do interior Solar. O painel a) mostra que o caminho das ondas acústicas está ligado ao aumento da velocidade do som com a profundidade até o alcance do ponto de retorno interior (círculos pontilhados). As ondas correspondem a modos com frequência $3000 \mu \mathrm{Hz}$ e $l=75,25,20$ e 2. A linha passando pelo centro ilustra um modo radial. O painel b) mostra o caminho das ondas de gravidade correspondentes ao modo com frequência $190 \mu H z$ e $l=5$. Créditos: Cunha et al. (2007).

Nessa aproximação, a dinâmica do modo p é determinada somente pela variação da velocidade do som com $r$. Assim, esses modos são ondas acústicas, tendo a pressão como força restauradora.

\subsubsection{Modos g}

Para modos de frequência baixa, $\omega \ll S_{l}$ na maior parte da estrela e o modo é oscilatório na região aproximadamente determinada por $\omega<N$. Assim, para esse caso $K$ é

$$
K(r) \simeq \frac{1}{\omega^{2}}\left(N^{2}-\omega^{2}\right) \frac{l(l+1)}{r^{2}} ;
$$

dessa forma, a dinâmica é dominada pela variação da frequência de empuxo $N$ com $r$. Os modos são ondas de gravidade aprisionadas, onde o empuxo de Arquimedes é a força restauradora.

Como pode ser visualizado na Figura 2.2b, os modos g podem ser ilustrados como a propagração de ondas de gravidade. No caso considerado, caso do Sol, os modos g estão aprisionados entre o centro e a base do envelope convectivo. 


\subsection{Teoria Assimptótica}

$\mathrm{Na}$ seção anterior, foram discutidas algumas propriedades qualitativas dos modos de oscilação estelar. Porém, para a investigação ser completa, é necessário resolver o sistema de equações de oscilação, seja através de cálculo numérico ou por análise assimptótica. Os dois métodos se complementam, o primeiro sendo apropriado para modos de $\mathbf{n}$ pequeno e intermediário e o outro para $\mathbf{n}$ grande (Unno et al., 1989). Nessa seção, as propriedades gerais das oscilações não-radiais estelares serão analisadas a partir do método assimptótico.

Fazendo a transformação de variáveis

$$
\tilde{\xi} \equiv r^{2} \xi_{r} \exp \left(-\int_{0}^{r} \frac{g}{c^{2}} d r\right)
$$

$\mathrm{e}$

$$
\tilde{\eta} \equiv \frac{p^{\prime}}{p} \exp \left(-\int_{0}^{r} \frac{N^{2}}{g} d r\right)=\omega^{2} r \xi_{r} \exp \left(-\int_{0}^{r} \frac{N^{2}}{g} d r\right)
$$

nas equações 2.74 e 2.75 da aproximação de Cowling, obtém-se

$$
\frac{d \tilde{\xi}}{d r}=h(r) \frac{r^{2}}{c^{2}}\left(\frac{S_{l}^{2}}{\omega^{2}}-1\right) \tilde{\eta}
$$

e

$$
\frac{d \tilde{\eta}}{d r}=\frac{1}{r^{2} h(r)}\left(\omega^{2}-N^{2}\right) \tilde{\xi}
$$

onde

$$
h(r) \equiv \exp \left[\int_{0}^{r}\left(\frac{N^{2}}{g}-\frac{g}{c^{2}}\right) d r\right]>0 .
$$

Combinando as duas equações para eliminar $\tilde{\xi}$ ou $\tilde{\eta}$, fica

$$
\frac{d^{2} \tilde{\xi}}{d r^{2}}-\frac{d \ln |P|}{d r} \frac{d \tilde{\xi}}{d r}-P Q \tilde{\xi}=0
$$

ou

$$
\frac{d^{2} \tilde{\eta}}{d r^{2}}-\frac{d \ln |Q|}{d r} \frac{d \tilde{\eta}}{d r}-P Q \tilde{\eta}=0
$$

onde

$$
P(r)=\frac{r^{2}}{c^{2}}\left(\frac{S_{l}^{2}}{\omega^{2}}-1\right) h(r)
$$

e

$$
Q(r)=\frac{1}{r^{2}}\left(\omega^{2}-N^{2}\right) h(r)^{-1}
$$


Deve-se perceber que um valor zero de $P(r)$ causa irregularidades em 2.96 e não em 2.97, e o oposto é válido para $Q(r)$, tornando essas equações complementares uma da outra. Para o caso limite em que $\omega^{2} \gg N^{2}$ a equação 2.97 leva a uma equação do tipo Sturm-Liouville,

$$
\frac{d^{2} \tilde{\eta}}{d r^{2}}+\frac{d \ln \left(r^{2} h\right)}{d r} \frac{d \tilde{\eta}}{d r}-\frac{l(l+1)}{r^{2}} \tilde{\eta}=-\frac{\omega^{2}}{c^{2}} \tilde{\eta},
$$

e para o caso limite em que $\omega^{2} \ll N^{2}$ a equação fica

$$
\frac{d^{2} \tilde{\eta}}{d r^{2}}+\frac{d}{d r} \ln \left(\frac{r^{2} h}{N^{2}}\right) \frac{d \tilde{\eta}}{d r}-\frac{N^{2}}{c^{2}} \tilde{\eta}=-\frac{1}{\omega^{2}} \frac{l(l+1) N^{2}}{r^{2}} \tilde{\eta} .
$$

De maneira similar, a equação 2.96 tende ao tipo Sturm-Liouville

$$
\frac{d^{2} \tilde{\xi}}{d r^{2}}+\frac{d}{d r} \ln \left(\frac{c^{2}}{r^{2}} h^{-1}\right) \frac{d \tilde{\xi}}{d r}-\frac{N^{2}}{c^{2}} \tilde{\xi}=-\frac{\omega^{2}}{c^{2}} \tilde{\xi}
$$

$\mathrm{Ou}$

$$
\frac{d^{2} \tilde{\xi}}{d r^{2}}+\frac{d}{d r} \ln \left(h^{-1}\right) \frac{d \tilde{\xi}}{d r}-\frac{l(l+1)}{r^{2}} \tilde{\xi}=-\frac{1}{\omega^{2}} \frac{l(l+1) N^{2}}{r^{2}} \tilde{\xi},
$$

para os casos onde $\omega^{2} \gg S_{l}^{2}$ ou $\omega^{2} \ll S_{l}^{2}$, respectivamente. Como nas equações $2.100 \mathrm{e}$ 2.102, $\omega^{2}$ é proporcional a $\omega^{2} / c^{2}$, as suas soluções levam a ondas acústicas. Já para as equações 2.101 e 2.103, a frequência é proporcional a $N^{2} k_{h}^{2} / \omega^{2}$ e as soluções são ondas de gravidade.

Introduzindo novas variáveis $v \equiv \tilde{\xi}|P|^{-1 / 2} \rho_{c}^{1 / 2}$ e $w \equiv \tilde{\eta}|Q|^{-1 / 2} \rho_{c}^{1 / 2}$, onde $\rho_{c}$ é a densidade estelar central, as equações podem ser reduzidas a equações de turning point,

$$
\frac{d^{2} v}{d r^{2}}+\left[k_{r}^{2}-f(P)\right] v=0
$$

$\mathrm{e}$

$$
\frac{d^{2} w}{d r^{2}}+\left[k_{r}^{2}-f(Q)\right] w=0
$$

com $k_{r}^{2}=-P Q$. Os pontos de retorno ocorrem onde $k_{r}^{2}-f(P)=0$ e $k_{r}^{2}-f(Q)=0$.

A solução da equação será dada em termos de funções de Airy dividindo o interior estelar em regiões com apenas um ponto de retorno. Depois, utiliza-se a forma assimptótica da função de Airy para unir essas regiões e obter a autofunção completa. A equação 2.104 é usada para obter a solução para os pontos singulares da equação 2.105 onde $\omega^{2}=S_{l}^{2}$ e a 2.105 é usada para $\omega^{2}=N^{2}$.

Para o caso de frequências altas, uma região de propagação, contendo um ponto de retorno $r_{a}$, se extende de $0 \leqslant r \leqslant r_{b}$, onde $r_{b}$ é outro ponto de retorno. É interessante 
introduzir a transformação de Liouville, que transforma as variáveis $(w, r)$ para $(W, \zeta)$

$$
\zeta \equiv\left(\frac{d r}{d \zeta}\right)^{2}\left[k_{r}^{2}-f(Q)\right] \simeq\left(\frac{d r}{d \zeta}\right)^{2} k_{r}^{2}
$$

e

$$
W \equiv\left(\left|\frac{d r}{d \zeta}\right|\right)^{-1 / 2} w
$$

E então $W$ satisfaz

$$
\frac{d^{2} W}{d \zeta^{2}}+\left[\zeta-f\left(\frac{d r}{d \zeta}\right)\right] W=0
$$

onde $f(x)$ é uma função regular definida como

$$
f(x) \equiv|x|^{1 / 2} \frac{d^{2}|x|^{-1 / 2}}{d r^{2}} .
$$

Para uma primeira aproximação na qual $f(d r / d \zeta)$ é negligenciado, $W$ é representado por

$$
W=a A i(\zeta)+b B i(\zeta)
$$

onde $A i(\zeta)$ e $B i(\zeta)$ são funções de Airy de primeiro tipo e de segundo tipo, respectivamente, que são relacionadas a funções de Bessel de ordem 1/3 como

$$
\begin{aligned}
A i(\zeta) & =\frac{1}{3}\left[\zeta^{1 / 2} J_{-1 / 3}\left(\frac{2}{3} \zeta^{3 / 2}\right)+\zeta^{1 / 2} J_{1 / 3}\left(\frac{2}{3} \zeta^{3 / 2}\right)\right] \\
B i(\zeta) & =\frac{1}{\sqrt{3}}\left[\zeta^{1 / 2} J_{-1 / 3}\left(\frac{2}{3} \zeta^{3 / 2}\right)-\zeta^{1 / 2} J_{1 / 3}\left(\frac{2}{3} \zeta^{3 / 2}\right)\right]
\end{aligned}
$$

e $a, b$ são constantes. Para altos valores de $|\zeta|$, as funções de Airy tomam a forma assimptótica, dada por

$$
\begin{aligned}
& A i(\zeta) \sim \frac{1}{2 \sqrt{\pi}}(-\zeta)^{-1 / 4} \exp \left[-\frac{2}{3}(-\zeta)^{3 / 2}\right] \\
& B i(\zeta) \sim \frac{1}{\sqrt{\pi}}(-\zeta)^{-1 / 4} \exp \left[\frac{2}{3}(-\zeta)^{3 / 2}\right]
\end{aligned}
$$

$\operatorname{para} \zeta<0, \mathrm{e}$

$$
\begin{aligned}
& A i(\zeta) \sim \frac{1}{\sqrt{\pi}} \zeta^{-1 / 4} \cos \left[\frac{2}{3} \zeta^{3 / 2}-\frac{\pi}{4}\right] \\
& B i(\zeta) \sim-\frac{1}{\sqrt{\pi}} \zeta^{-1 / 4} \sin \left[\frac{2}{3} \zeta^{3 / 2}-\frac{\pi}{4}\right]
\end{aligned}
$$

para $\zeta>0$. A combinação das equações 2.106, 2.107 e 2.110 leva a função $w$ a forma

$$
w=\left|k_{r}\right|^{-1 / 2}\left(\left|\frac{3}{2} \int_{r_{a}}^{r}\right| k_{r}|d r|\right)^{1 / 6}[a A i(\zeta)+b B i(\zeta)]
$$


onde

$$
\zeta=\operatorname{sgn}\left(k_{r}^{2}\right)\left(\left|\frac{3}{2} \int_{r_{a}}^{r}\right| k_{r}|d r|\right)^{2 / 3}
$$

e o símbolo $\operatorname{sgn}(x)$ significa a função sinal da função x. Substituindo as equações 2.112 e 2.113 na equação 2.114, obtém-se a forma asimptótica da função $w$, definido na equação 2.105 ,

$$
w \sim \begin{cases}\frac{a}{2 \sqrt{\pi}} \frac{1}{\sqrt{\kappa}} \exp \left(-\int_{r}^{r_{a}} \kappa d r\right)+\frac{b}{\sqrt{\pi}} \frac{1}{\sqrt{\kappa}} \exp \left(\int_{r}^{r_{a}} \kappa d r\right), & \text { para } r \ll r_{a} \\ \frac{a}{\sqrt{\pi}} \frac{1}{\sqrt{k_{r}}} \cos \left(\int_{r_{a}}^{r} k_{r} d r-\frac{\pi}{4}\right)-\frac{b}{\sqrt{\pi}} \frac{1}{\sqrt{k_{r}}} \sin \left(\int_{r_{a}}^{r} k_{r} d r-\frac{\pi}{4}\right), & \text { para } r_{a} \ll r,\end{cases}
$$

com $\kappa^{2} \equiv-k_{r}^{2}$. O coeficiente $b$ tem que ser igual a zero para a função ser regular em $r=0$. Da mesma forma, da equação 2.94 pode ser escrita como

$$
v=\operatorname{sgn}(Q)\left|k_{r}\right|^{-1}\left(\frac{d w}{d r}+\frac{1}{2} \frac{d \ln |Q|}{d r} w\right) \simeq \operatorname{sgn}(Q)\left|k_{r}\right|^{-1} \frac{d w}{d r} .
$$

Substituindo a equação 2.116 com $b=0$ em 2.117, obtém-se a forma assimptótica para a função $v$ para o caso onde $r \ll r_{a}$

$$
v \sim-\frac{a}{\sqrt{\pi}} \frac{1}{\sqrt{k_{r}}} \sin \left(\int_{r_{a}}^{r} k_{r} d r-\frac{\pi}{4}\right) .
$$

De maneira similar, a equação 2.104 é utilizada para obter a função $v$ para $r_{a}<r$. A forma assimptótica da função $v$, definido na equação 2.104, para este intevalo fica

$$
v \sim \begin{cases}\frac{c}{\sqrt{\pi}} \frac{1}{\sqrt{k_{r}}} \cos \left(\int_{r}^{r_{b}} k_{r} d r-\frac{\pi}{4}\right)-\frac{d}{\sqrt{\pi}} \frac{1}{\sqrt{k_{r}}} \sin \left(\int_{r}^{r_{b}} k_{r} d r-\frac{\pi}{4}\right), & \text { para } r_{a} \ll r \ll r_{b} \\ \frac{c}{2 \sqrt{\pi}} \frac{1}{\sqrt{\kappa}} \exp \left(-\int_{r_{b}}^{r} \kappa d r\right)+\frac{d}{\sqrt{\pi}} \frac{1}{\sqrt{\kappa}} \exp \left(\int_{r_{b}}^{r} \kappa d r\right), & \text { para } r_{b} \ll r,\end{cases}
$$

com $c$ e $d$ constantes. A forma assimptótica se reduz a

$$
w \sim-\frac{c}{\sqrt{\pi}} \frac{1}{\sqrt{k_{r}}} \sin \left(\int_{r}^{r_{b}} k_{r} d r-\frac{\pi}{4}\right)-\frac{d}{\sqrt{\pi}} \frac{1}{\sqrt{k_{r}}} \cos \left(\int_{r}^{r_{b}} k_{r} d r-\frac{\pi}{4}\right)
$$

para $r_{a} \ll r \ll r_{b}$. Os dois tipos de expressões assimptóticas para $v$ e $w$ tem que concordar entre si. Eliminando $d=0$ (mesmo caso que $b=0$ ), das equações 2.118 e 2.119 , obtém-se

$$
c=-a \sin \left(\int_{r_{a}}^{r_{b}} k_{r} d r-\frac{\pi}{2}\right) .
$$

De maneira similar,

$$
d=-a \cos \left(\int_{r_{a}}^{r_{b}} k_{r} d r-\frac{\pi}{2}\right) .
$$

Na expressão de $v$ para $r_{b} \ll r$ dada pela equação 2.119 o termo com o coeficiente $c$ no lado direito representa a onda evanescente vindo do interior estelar. Mas o segundo termo 
com o coeficiente $d$ representa a onda vindo de fora da estrela e esta tem que tender a zero. Assim, $d=0$ como as condições de contorno para 2.104 e 2.105. Porém, $d=0$ quando

$$
\int_{r_{a}}^{r_{b}} k_{r} d r=n \pi
$$

com $n$ inteiro. Essa condição para as auto-oscilações corresponde a quantização de BohrSommerfeld em mecânica quântica.

Os coeficientes $b$ e $d$ são iguais a zero e o coeficiente $c$ é igual a $(-1)^{n} a$ como resultado da última equação. Existem $n$ nodos da função $v$, que corresponde a nodos da função $\xi_{r}$, em uma zona de propagação. Tais automodos são classificados como modos $p_{n}$ pelo número de modos da função $\xi_{r}$.

A regra de quantização leva a autofrequências $\omega_{n}$. O ponto de retorno externo $\left(r_{b}\right)$ ocorre, em muitos casos, muito perto da fotosfera. Então $r_{b}$ pode ser aproximado para $R$. Estimando a quantidade $k_{r}$ para

$$
k_{r} \approx\left\{\begin{array}{l}
\omega / c ; r_{a} \ll r \ll R \ll r_{b} \\
c^{-1}\left(\omega^{2}-S_{l}^{2}\right)^{1 / 2}, \quad \text { para } r \sim r_{a},
\end{array}\right.
$$

e substituindo na equação de quantização, obtém-se

$$
\int_{r_{a}}^{r *} c^{-1}\left(\omega^{2}-S_{l}^{2}\right)^{1 / 2} d r+\omega \int_{r *}^{R} c^{-1} d r=n \pi
$$

onde $r *$ é a distância radial onde a velocidade do som é quase igual ao valor central $c_{0}$. Assume-se que, para $r>r *$ o raio $S_{l}^{2} / \omega^{2}$ pode ser negligenciado em comparação com a unidade. Integrando o primeiro termo do lado esquerdo dessa equação levará a

$$
\omega \int_{r_{a}}^{r *} c^{-1}\left[1-\frac{l(l+1) c^{2}}{\omega^{2} r^{2}}\right]^{1 / 2} d r \simeq \omega c_{0}^{-1}\left[\left(r *^{2}-r_{a}^{2}\right)^{1 / 2}-\omega^{-1}[l(l+1)]^{1 / 2} c_{0} \cos ^{-1}\left(\frac{r_{a}}{r *}\right)\right] .
$$

No caso de modos p com $\mathbf{n} \gg 1$ com $\mathbf{l} \sim 1$, o ponto de retorno interno $r_{a}$ é próximo ao centro da estrela, fazendo assim $r_{a} \simeq 0$ para

$$
\omega \int_{r_{a}}^{r *} c^{-1}\left[1-\frac{l(l+1) c^{2}}{\omega^{2} r^{2}}\right]^{1 / 2} d r \simeq \omega \int_{0}^{r *} c^{-1} d r-[l(l+1)]^{1 / 2} \frac{\pi}{2} .
$$

Adicionando a segunda integral do lado esquerdo da equação 2.125, obtém-se a autofrequência $\omega_{n l}$ para o modo $p_{n}$ igual a

$$
\omega_{n l} \approx 2 \pi \nu_{0}\left(n+\frac{l}{2}+\alpha\right)
$$




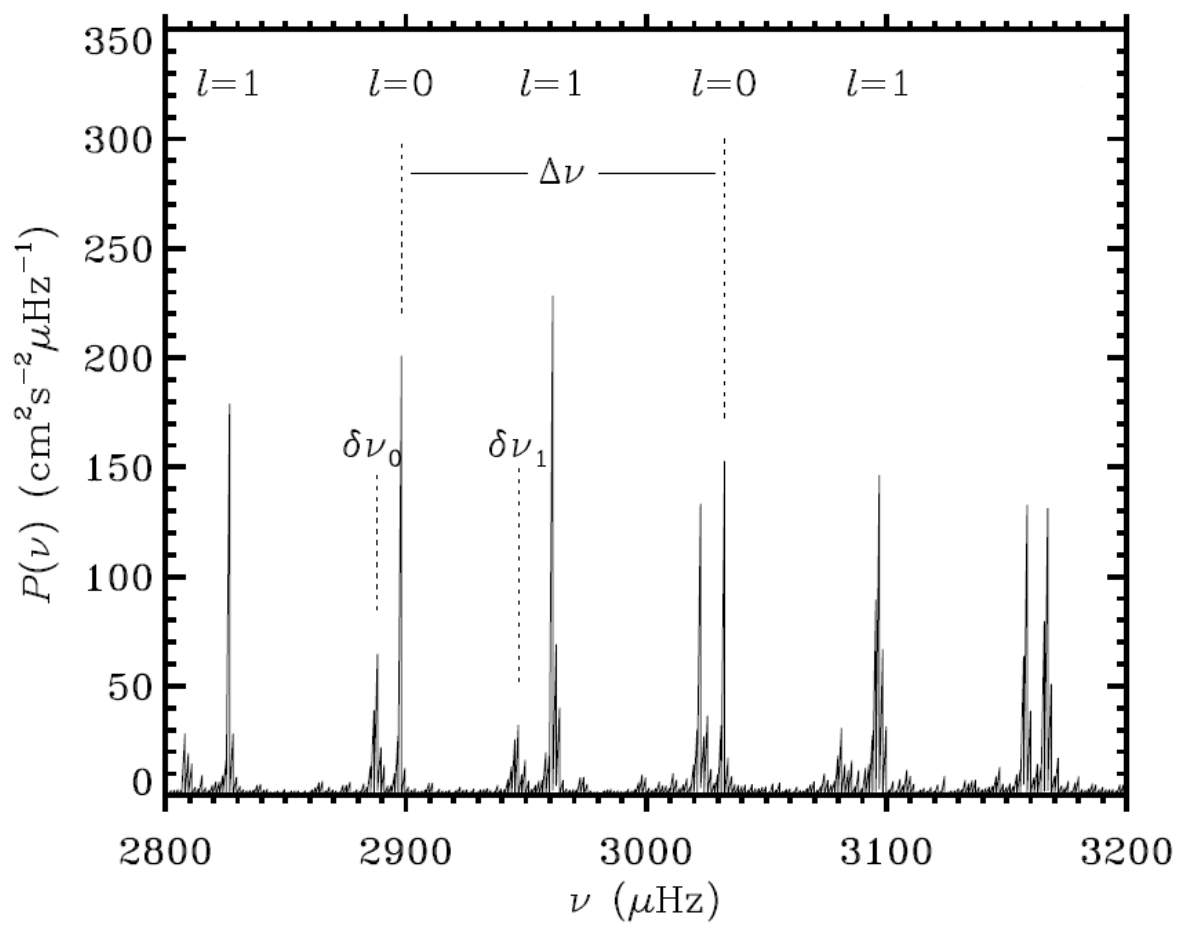

Figura 2.3: Uma ampliação do espectro de potências de oscilações solares obtido com observações Doppler da luz integrada do disco Solar. Alguns modos foram identificados com seu grau 1. O espectro apresenta um espaçamento regular previsto pela teoria assimptótica. A "grande" e "pequena" separação estão indicadas. Créditos: Christensen-Dalsgaard (2003).

onde

$$
\nu_{0} \equiv\left[2 \int_{0}^{R} \frac{1}{c} d r\right]^{-1}
$$

sendo $\alpha$ uma constante. Essa formula é válida para um modo p de alta ordem e baixo grau (Tassoul, 1980). Ela prevê um espaçamento uniforme entre modos de mesmo grau. Esse espaçamento entre modos de ordem consecutiva é conhecido como "grande separação" em frequência (large frequency separation - $\Delta \nu_{n l}$ ). Ela prevê também uma degenerescência entre modos com grau de mesma paridade $\nu_{n l} \simeq \nu_{n-1} l+2$ e leva a outra propriedade chamada "pequena separação" em frequência ( small frequency separation - $\delta \nu_{n l}$ ). A Figura 2.3 mostra uma ampliação dp espectro solar com a característica da "grande" e "pequena" separação indicadas. É evidente que a separação depende do grau; isso pode ser usado para identificar os modos observados, como foi feito no caso do Sol (e.g. Christensen-Dalsgaard e Gough, 1982).

De maneira similar, encontra-se para o caso de modos de baixa frequência, modos g, 
obtém-se

$$
\omega_{n} \simeq \frac{[l(l+1)]^{1 / 2}}{n \pi} \int_{r_{a}}^{r_{b}} \frac{N}{r} d r .
$$

No entanto, essa fórmula para $\omega_{n}$ é formal já que o ponto de retorno depende fortemente da frequência em casos usuais. Para o caso de modos de gravidade dom alto $\mathbf{n}, r_{a}$ e $r_{b}$ correspondem a parte externa da camada convectiva e superfície estelar, respectivamente, e são praticamente independentes de $\omega_{n}$.

\subsection{Rotação na Estrela}

A rotação introduz um eixo de simetria no sistema e levanta a degenerescência do problema fazendo com que o sistema se torne dependente do número quântico $\mathbf{m}$ também.

Considere-se o sistema de coordenadas $\left(r^{\prime}, \theta^{\prime}, \phi^{\prime}\right)$ relacionado ao sistema inercial por $\left(r^{\prime}, \theta^{\prime}, \phi^{\prime}\right)=(r, \theta, \phi-\Omega t)$. Neste caso, as perturbações serão dependentes de $t$ e $\phi^{\prime}$ como

$$
\cos \left(m \phi^{\prime}-\omega_{0} t\right)
$$

e a perturbação será dependente com relação ao sistema inercial da seguinte forma

$$
\cos \left(m \phi+\omega_{m} t\right)
$$

onde

$$
\omega_{m}=\omega_{0}+\Omega t .
$$

Assim, um observador no sistema inercial encontra que a frequência está separada (split) uniformemente com $\mathbf{m}$.

Porém, esse tratamento está incompleto. Mesmo considerando uma rotação uniforme, é necessário levar em conta a força de Coriolis na separação das frequências (Cowling e Newing, 1949). Contudo, normalmente o efeito da força de Coriolis é pequeno e a equação 2.133 será uma boa aproximação se $\Omega$ for substituida pela média em posição da velocidade angular (Christensen-Dalsgaard, 2003).

Introduzindo um campo de velocidades nas equações de perturbação e separando as dependências com o tempo como $\exp (-i \omega t)$, a equação de movimento do gás estelar fica

$$
-\omega^{2} \rho_{0} \overrightarrow{\delta r}-2 i \omega \rho_{0}\left(\overrightarrow{v_{0}} \cdot \vec{\nabla}\right) \overrightarrow{\delta r}=-\vec{\nabla} p^{\prime}+\rho_{0} \vec{g}^{\prime}+\rho^{\prime} \overrightarrow{g_{0}}
$$


Essa equação pode ser escrita da forma

$$
\omega^{2} \overrightarrow{\delta r}=\mathcal{F}(\overrightarrow{\delta r})+\delta \mathcal{F}(\overrightarrow{\delta r})
$$

onde

$$
\delta \mathcal{F}(\overrightarrow{\delta r})=-2 i \omega\left(\overrightarrow{v_{0}} \cdot \vec{\nabla}\right) \overrightarrow{\delta r}
$$

E então, uma perturbação $\delta \omega$ causada por $\delta \mathcal{F}$ em $\omega$ pode ser obtida por análise de perturbação de primeira ordem, na forma

$$
\delta \omega=-i \frac{\int_{V} \rho_{0} \delta \vec{r} * \cdot\left(\overrightarrow{v_{0}} \cdot \vec{\nabla}\right) \overrightarrow{\delta r} d V}{\int_{V} \rho_{0}|\overrightarrow{\delta r}|^{2} d V} .
$$

\subsubsection{Splitting Rotacional}

O splitting rotacional é a separação em frequência dos modos originalmente degenerados em m. Para correção em primeira ordem de $\omega_{n l m}$, a força de Coriolis domina e o termo de correção é dado por

$$
\delta \omega_{n l m}=m \int_{0}^{R} \int_{0}^{\pi} K_{n l m}(r, \theta) \Omega(r, \theta) r d r d \theta
$$

onde $K_{n l m}$ é o kernel rotacional. Assumindo rotação shellular $\Omega(r)=\Omega_{s}\left[1+\eta_{0}(r)\right]$, o kernel rotacional fica

$$
K(r)=\frac{\xi_{r}^{2}-2 \xi_{r} \xi_{h}+(\Lambda-1) \xi_{r}^{2}}{\xi_{r}^{2}+\Lambda \xi_{h}^{2}} r^{2} \rho_{0},
$$

onde $\Lambda=l(l+1)$.

Dessa forma, o splitting rotacional é definido por

$$
S_{m}=\frac{\omega_{n l m}-\omega_{n l-m}}{2 m} .
$$

No caso de rotação uniforme, ele simplifica para

$$
S_{m}=m \Omega \int_{0}^{R} K(r) d r
$$

E no caso específico de rotação shellular, o splitting fica (Suárez et al., 2006)

$$
S_{m}=m \Omega_{s}\left(C_{L}-1-J_{0}\right),
$$

onde $C_{L}$ é a constante de Ledoux

$$
C_{L}=\frac{1}{I_{0}} \int_{0}^{R}\left[2 y_{01} z_{0}+z_{0}^{2}\right] \rho_{0} r^{4} d r
$$


onde $I_{0}$ é

$$
I_{0}=\int_{0}^{R}\left[y_{01}^{2}+\Lambda z_{0}^{2}\right] \rho_{0} r^{4} d r
$$

e $J_{0}$ é

$$
J_{0}=\frac{1}{I_{0}} \int_{0}^{R} \eta_{0}(r)\left[y_{01}^{2}+\Lambda z_{01}-2 y_{01} z_{01}-z_{01}^{2}\right] \rho_{0} r^{4} d r
$$

\subsubsection{Assimetrias no Splitting Rotacional}

Ao considerar rotação não-uniforme, as correções de segunda ordem na frequência se tornam importantes. Contudo, diferentemente das correções de primeira ordem, as correções de segunda ordem para pares de mesmo $|m|$ não são simétricas, levando a assimetrias que podem ser obtidas para um dado 1 por

$$
A_{m}=\omega_{l,-m}+\omega_{l,+m}-2 \omega_{l, 0}=-\frac{6 m^{2}}{4 \Lambda-3} \frac{\bar{\Omega}^{2}}{\omega_{0}} \mathcal{I}_{c},
$$

onde, para modos p de frequência alta, $\mathcal{I}_{c}$ pode ser dado com boa aproximação por

$$
\mathcal{I}_{c} \sim \sigma_{0}^{2}\left\langle S_{2}\right\rangle
$$

para $\sigma_{0}^{2}=\omega_{0}^{2} /\left(G M / R^{3}\right)$ e $\left\langle S_{2}\right\rangle$ é a média ponderada das perturbações da estrutura (Suárez et al., 2006).

As assimetrias e splittings de um modo com $\mathbf{l} \neq 0$ podem trazer importantes informações sobre a rotação estelar e são utilizadas com esse propósito na análise feita nesse trabalho.

\subsection{Mecanismos de Excitação das Oscilações Estelares}

As amplitudes e tempo de vida dos modos de oscilação são diretamente relacionados com os mecanismo de amortecimento e excitação. Ao considerar oscilações adiabáticas, nenhuma informação é obtida sobre o amortecimento ou excitação dos modos porque as equaçãos são conservativas e não envolvem a troca de energia entre as oscilações e o fluxo de energia no modelo de equilíbrio. Cálculos levando em conta efeitos não adiabáticos investigaram a estabilidade linear das oscilações estelares. Isso é determinado pela parte imaginária $\omega_{i}$ da frequência complexa $\omega=\omega_{r}+i \omega_{i}$, e modos com $\omega_{i}$ positivos são instáveis (Christensen-Dalsgaard, 2002). 


\subsubsection{Mecanismo do tipo Motor Térmico}

Considerando agora uma perturbação $\delta \mathcal{F}$ como o desvio das oscilações adiabáticas na equação do momento, esta pode ser escrita como

$$
\omega^{2} \overrightarrow{\delta r}=\mathcal{F}_{a d}(\overrightarrow{\delta r})+\delta \mathcal{F}(\overrightarrow{\delta r})
$$

com

$$
\mathcal{F}_{a d}(\overrightarrow{\delta r})=\frac{1}{\rho} \vec{\nabla} p_{a d}^{\prime}-\vec{g}^{\prime}-\frac{\rho^{\prime}}{\rho} \vec{g}
$$

$\mathrm{e}$

$$
\delta \mathcal{F}(\overrightarrow{\delta r})=\frac{i}{\omega \rho} \nabla\left[\left(\Gamma_{3}-1\right)(\rho \epsilon-\vec{\nabla} \cdot \vec{F})^{\prime}\right] .
$$

Assim, os efeitos na frequência devido a esses desvios na adiabaticidade ficam

$$
\delta \omega=\frac{i}{2 \omega^{2}} \frac{\int_{V} \frac{\delta \rho *}{\rho}\left(\Gamma_{3}-1\right)(\rho \epsilon-\vec{\nabla} \cdot \vec{F})^{\prime} d V}{\int_{V} \rho|\overrightarrow{\delta r}|^{2} d V} .
$$

Essa expressão levanta o aspecto de motor térmico da excitação, onde $\delta(\rho \epsilon-\vec{\nabla} \cdot \vec{F})$ é a perturbação no aquecimento; regiões onde o aquecimento é positivo por compressão contribuem para a excitação do modo, como esperado para um motor térmico. Obviamente, outras regiões podem ter fase contrária e a excitação do modo requer que a região contribua para a parte dominante da integral.

O termo em $\delta(\rho \epsilon)$ tipicamente contribue para a excitação, já que a compressão leva a uma alta temperatura e portanto, a uma alta taxa de geração de energia. Usando $\epsilon=\epsilon(\rho, T)$ é fácil notar que

$$
(\rho \epsilon)^{\prime}=\rho \epsilon\left[\epsilon_{T} \frac{T^{\prime}}{T}+\left(\epsilon_{\rho}+1\right) \frac{\rho^{\prime}}{\rho}\right],
$$

onde

$$
\epsilon_{T}=\left(\frac{\partial \ln \epsilon}{\partial \ln T}\right)_{\rho}, \quad \epsilon_{\rho}=\left(\frac{\partial \ln \epsilon}{\partial \ln \rho}\right)_{T} .
$$

Similarmente, a perturbação no fluxo pode ser determinada da aproximação da difusão, assumindo que não há nenhuma outra contribuição para a distribuição de energia. O resultado é

$$
\vec{F}^{\prime}=\left[\left(3-\kappa_{T}\right) \frac{T^{\prime}}{T}-\left(1+\kappa_{\rho}\right) \frac{\rho^{\prime}}{\rho}\right] F_{r} \overrightarrow{a_{r}}-\frac{4 a \bar{c} T^{3}}{3 \kappa \rho} \vec{\nabla} T^{\prime}
$$


onde

$$
\kappa_{T}=\left(\frac{\partial \ln \kappa}{\partial \ln T}\right)_{\rho}, \quad \kappa_{\rho}=\left(\frac{\partial \ln \kappa}{\partial \ln \rho}\right)_{T},
$$

e $F_{r}$ é o fluxo radiativo de equilíbrio. Independentemente da suposição de adiabaticidade, $\rho^{\prime}$ pode ser obtido da equação da continuidade como

$$
\frac{\delta \rho}{\rho}=-\vec{\nabla} \cdot \overrightarrow{\delta r}, \quad \rho^{\prime}=\delta \rho-\xi_{r} \frac{d \rho}{d r} .
$$

E a perturabação na temperatura pode ser obtida de

$$
\frac{\delta T}{T}=\left(\Gamma_{3}-1\right) \frac{\delta \rho}{\rho}, \quad T^{\prime}=\delta T-\xi_{r} \frac{d T}{d r} .
$$

Essa aproximação é conhecida como aproximação quasi-adiabática. Ela é baseada na suposição de que a perturbação é pequena; isso, porém, não é verdade para regiões próximas à superfície, onde os efeitos não-adiabáticos tornam-se importantes. No entanto ela fornece indicações sobre a estabilidade dos modos.

Considerando um caso simples, onde a não-adiabaticidade é dominada pela geração de energia, encontra-se

$$
\delta \omega=\frac{i}{2 \omega^{2}} \frac{\int_{V}\left|\frac{\delta \rho}{\rho}\right|^{2}\left(\Gamma_{3}-1\right)\left[\epsilon_{\rho}+1+\left(\Gamma_{3}-1\right) \epsilon_{T}\right] \rho \epsilon d V}{\int_{V} \rho|\overrightarrow{\delta r}|^{2} d V} .
$$

Como $\epsilon_{T}$ e $\epsilon_{\rho}$ são positivos e $\Gamma_{3} \simeq 5 / 3$, é óbvio que as integrais nessa equação são positivas. Com a hipótese assumida de que a dependência no tempo é exp $(-i \omega t)$, isso corresponde a um crescimento na amplitude de oscilação, ou seja, a uma instabilidade do modo.

A natureza do modo é simples. O gás se aquece devido a compressão e isso, juntamente com o aumento na densidade, causa um aumento na liberação de energia; isso aumenta a tendência do gás para expandir até o equilíbrio novamente; na expansão o gás fica mais frio e menos denso e portanto a energia produzida diminui; isso aumenta a tendência do gás colapsar em torno do equilíbrio; ambos os efeitos aumentam a amplitude da oscilação.

Essa análise determina qual dos modos são linearmentes instáveis e portanto, tem tendência a crescer, mas não diz nada a respeito da amplitude resultante. Formalmente, a amplitude cresce exponencialmente e deve existir obviamente outro mecanismo para limitálas a valores finitos. Porém, ainda falta conhecimento sobre qual é o fenômeno físico que limita as amplitudes em estrelas multiperiódicas de baixa amplitude (Cunha et al., 2007). 
Uma tentativa para remediar a situação são os modos radiais computados por Smolec e Moskalik (2007), que encontraram que a saturação de diversos mecanismos de excitação por vários modos acústicos pode levar a previsão das amplitudes na mesma escala observada.

\subsubsection{Pulsações Excitadas Estocasticamente}

Aparentemente, modos em estrelas de baixa temperatura efetiva são geralmente estáveis. Os modos excitados observados são atribuídos a forças estocásticas perto da superfície de convecção. De fato, fluxos turbulentos com velocidades próximas à do som são uma fonte eficiente para a radiação acústica e é provável que isso excite os modos normais da estrela nas amplitudes observadas (Christensen-Dalsgaard, 2003).

Batchelor (1953) considerou o problema geral de osciladores amortecidos excitados estocaticamente. O espectro de potências resultante para um modo de frequência $\omega_{0}$ e amortecimento $\eta$ é dado aproximadamente por

$$
\langle P(\omega)\rangle \simeq \frac{1}{4 \omega_{0}^{2}} \frac{\left\langle P_{f}(\omega)\right\rangle}{\left(\omega-\omega_{0}\right)^{2}+\eta^{2}},
$$

onde $\left\langle P_{f}(\omega)\right\rangle$ é a espectro de potência médio da função força. Se o espectro da força é uma função da frequência que varia lentamente, o resultado é o espectro Lorentziano com o comprimento determinado pela taxa de amortecimento linear do modo.

Como resultado da natureza estocástica da excitação, a amplitude do modo observada varia com o tempo. Kumar et al. (1988) estudaram as propriedades estatística dessas variações. Se um modo é observado durante um intervalo de tempo curto comparado como tempo de amortecimento, a distribuição da energia é exponencial,

$$
p(E) d E=\langle E\rangle^{-1} \exp (-E /\langle E\rangle) d E,
$$

onde $\langle E\rangle$ é a energia média e a energia $E$ é proporcional ao quadrado da amplitude.

A função distribuição na equação 2.160 também define uma relação entre a amplitude média $\langle A\rangle$ e o desvio padrão $\sigma(A)$ que pode ser escrita como

$$
\sigma(A)=\left(\frac{4}{\pi}-1\right)^{1 / 2}\langle A\rangle \simeq 0,52\langle A\rangle .
$$

Como indicado nessa equação, dado um modelo para a potência da força estocástica (equação 2.159), esse mecanismo de excitação resulta em previsões definitivas da amplitude de oscilação. Isso pode ser avaliado com modelos para convecção como o modelo de mixing-length. 
É esperado que o mecanismo estocástico resulte na excitação de todos os modos em uma larga faixa de frequências, com a amplitude refletindo a dependência com a função força das frequências baixas. Essa propriedade é observada no Sol e em oscilações do tipo-Solar em outras estrelas.

\subsection{Convecção}

A descrição padrão para a convecção usada em modelos estelares é a teoria do comprimento de mistura de Böhm-Vitense 1958 (apud Kippenhahn e Weigert, 1990). Nessa teoria, o movimento convectivo é tratado como independente do tempo. Na falta de uma teoria rigorosa sobre o movimento adiabático baseada em primeiros princípios, as células convectivas são assumidas terem um livre caminho médio de $\alpha_{M L} H_{p}$, onde $H_{p}$ é a escala de altura da pressão local. O parâmetro de comprimento de mistura depende da física considerada no modelo e na formulação do MLT usada.

No contexto da evolução estelar, é de crucial importância quantificar a quantidade de matéria misturada na região central da estrela. Essa quantidade é derivada usualmente pelo critério de Schwarzschild, que assume que a convecção ocorre em regiões onde o gradiente de temperatura é menor que o gradiente radiativo. No entanto, de um ponto de vista físico, é bem improvável que os elementos de convecção parem abruptamente na franteira estabelecida pelo critério de Schwarzschild. Ao contrário, suas inércias fazem com que eles ultrapassem (overshoot) para a área estável onde o transporte de energia é radiativo. A quantidade de overshooting, no entanto, é bastante debatida. Por essa razão, é comum expressar como $d_{o v} H_{p}$, onde $d_{o v}$ é uma pequena fração de $\alpha_{M L}$ (Aerts et al., 2008). 
Capítulo 3

\section{Satélites}

A sismologia estelar teve um grande avanço com o desenvolvimento da sismologia espacial. Observações do espaço podem proporcionar conjuntos de dados longos e quase contínuos para um mesmo objeto, o que não é possível nas observações de solo devido à sucessão dia-noite. Além disso, um fotômetro com abertura moderada pode ser usado para controlar as oscilações com amplitudes em torno de 1 ppm, e com taxas de amostragem elevadas durante as observações é possível, em princípio, ter acesso a escalas de tempo características impossíveis de serem acessados em observações de solo.

Uma das missões pioneiras, como o satélite canadense MOST, não conseguiram chegar ao baixo nível de ruído exigido para a observação de oscilações do tipo solar em estrelas da sequência principal, mas ele forneceu dados de alta qualidade para um número considerável de outros tipos de estrelas pulsantes (Christensen-Dalsgaard, 2010). Já o satélite francoeuropeu-brasileiro CoRoT resultou em um avanço no estudo das oscilações do tipo solar, bem como para outros tipos de estrelas pulsantes clássicas. Uma das principais conquistas dele foi a possibilidade de observações quase contínuas por cinco meses. Observações ainda mais extensas estão sendo obtidas pela missão Kepler (NASA). Mesmo a análise do início do primeiro mês de dados de Kepler demonstrou uma qualidade surpreendente destes dados, e espera-se que, após uma fase de levantamento de um ano de duração, um número substancial de estrelas possa ser observado no período de pelo menos 3 anos e meio de missão.

Neste capítulo, são apresentados as principais características das missões espaciais dedicadas a sismologia estelar. Na seção 3.1 é abordado o satélite canadense MOST. Já na seção 3.2, o satélite CoRoT é discutido e na seção 3.3, a missão Kepler da NASA. 


\subsection{MOST}

\subsubsection{Objetivo da Missão Espacial}

O satélite MOST (Microvariability and Oscillations of STars, e.g. Walker et al., 2003) foi uma missão de fotometria estelar ultraprecisa de longa duração. Seu objetivo foi medir as variações de brilho das estrelas continuamente por 40 dias. A missão foi financiada pela Agência Espacial Canadense (CSA) e o instrumento desenvolvido pelo Departamento de Física e Astronomia da Universidade de British Columbia. Os detalhes da missão podem ser encontrados no site: http://www.astro.ubc.ca/MOST/ .

Ele foi lançado em junho de 2003 a bordo de um foguete russo de 3 estágios, e colocado em uma órbita polar a $820 \mathrm{~km}$ de altitude, permitindo a observação de um mesmo campo por até 60 dias ininterruptamente. Para observações de longa duração, o satélite ficava apontado inercialmente com precisão e estabilidade melhor do que meio minuto de arco.

A missão MOST teve como objetivo principal detectar fotometricamente o espectro de frequências de oscilações acústicas de baixo grau de estrelas do tipo solar e estrelas Ap. Isso incluiu a detecção e caracterização dessas oscilações, inclusive de estrelas mais velhas (pobres em metal) e com campos magnéticos, para explorar sismicamente suas estruturas e idades; a detecção de luz refletida de exoplanetas gigantes orbitando proximamente estrelas do tipo solar, para revelar seus tamanhos e composições atmosféricas; e detectar variações turbulentas em estrelas massivas evoluídas (Wolf-Rayet) para entender como elas injetam gás no meio interestelar.

O instrumento foi projetado para detectar variações no brilho das estrelas de pequenas partes por milhão - a amplitude das oscilações de 5 minutos do Sol observadas no brilho integrado. A sensibilidade fotométrica dele também permitiu detectar variações estocásticas em ventos estelares quentes, testando assim diversos mecanismos de condução e de turbulência propostos, e melhor estimando a contribuição dos ventos para o meio interestelar.

\subsubsection{Aspectos Gerais}

O microsatélite possuía um tamanho de 65 x 65 x $30 \mathrm{~cm}$, com massa estimada em $60 \mathrm{~kg}$. Ele era alimentado por painéis solares e orientado por um sistema de rodas de 


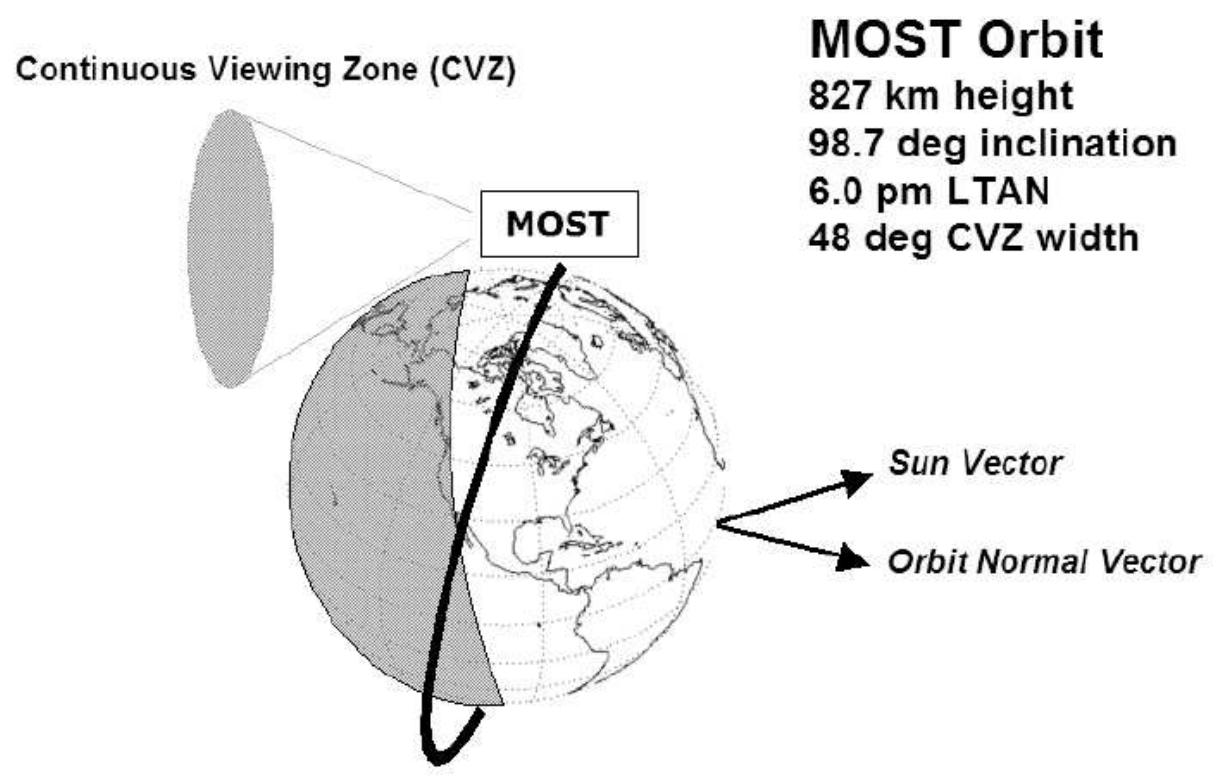

Figura 3.1: Esquema da órbita do satélite MOST com o campo de visão contínuo (CVZ).

Créditos: CSA (2011).

reação e torques magnéticos. O sistema de controle de altitude mantinha o telescópio apontando dentro de 10 segundos de arco do alvo desejado, 99\% das vezes. Sua órbita era heliossíncrona a cerca de $820 \mathrm{~m}$ de altitude.

A carga útil foi composta por um telescópio óptico com um espelho de apenas $15 \mathrm{~cm}$ de diâmetro, alimentando um detector CCD (Charge Coupled Device) com dispositivos Marconi duplos de transferência de quadro lado a lado. Um CCD foi utilizado para medições científicas e o outro lido a cada segundo para o acompanhamento de estrelas guias e estabilidade do satélite. O instrumento continha um único filtro de banda larga que selecionava a luz na faixa de comprimento de ondas 350-700 nm.

A câmera era equipada com uma matriz de microlentes de Fabry, que projetavam a imagem de estrelas alvo com grande estabilidade, o que consistiu no ponto fundamental para a precisão fotométrica do MOST. Por questão de baixo custo e alta confiabilidade, o instrumento não tinha partes móveis - a estrutura mantinha automaticamente o mesmo foco em um amplo intervalo de temperaturas e os tempos de exposição eram controlados por quadro de transferência rápida dos CCDs. Os CCDs eram refrigerados por um sistema de radiador passivo. 
Sua órbita permitia um campo de visão contínuo entre as declinações $-19^{\circ}$ e $+36^{\circ}$ no qual uma estrela alvo podia ser observada por até 60 dias ininterruptamente. A Figura 3.1 mostra um esquema da órbita do satélite com o campo de visão contínua (CVZ). O período do ano em que a estrela se encontrava no CVZ dependia da ascensão reta. Como referência, uma estrela com ascensão reta igual a $6 h 40 m$ e declinação de $+9^{\circ}$ estava centrada na CVZ no dia $1^{\circ}$ de janeiro de cada ano.

\subsection{CoRoT}

\subsubsection{Objetivo da Missão Espacial}

O projeto CoRoT (COnvection, ROtation and planetary Transits, e.g. Boisnard e Auvergne, 2006) é uma missão dedicada à astrofísica estelar e à procura de exoplanetas. Este satélite integra o programa de pequenos satélites da agência espacial francesa CNES em conjunto com a Agência Espacial Européia (ESA) e conta com a participação da Alemanha, Áustria, Bélgica, Espanha e Brasil. Os detalhes da missão podem ser encontrados no site: http://corot.oamp.fr/index.html.

O satélite foi colocado em órbita em dezembro de 2006 e operará até 2013. A missão apresenta dois programas científicos. Ambos os programas requerem observações longas ininterruptas com alta precisão fotométrica. Os programas são executadas simultaneamente em partes adjacentes do céu.

Um dos objetivos principais dele é estudar a estrutura de interiores estelares através da sismologia estelar. Durante a missão, o satélite permitirá que se estude as estruturas internas de milhares de estrelas para determinar suas massas, idade e composição química. Para isto, usa métodos de fotometria estelar com elevada precisão nas medidas das variações no fluxo luminoso recebido das estrelas sobre um longo período de tempo. As medidas são efetuadas na banda de 0,1 a $10 \mu \mathrm{Hz}$, cobrindo tanto modos acústicos, como modos de gravidade da maioria das estrelas. O programa central de sismologia é capaz de observar variações de luminosidade com a precisão de até $10^{-6}$ para estrelas mais brilhantes que magnitude 9,5. As observações de até 150 dias de duração resultam em uma resolução de $0,1 \mu H z$ no espaço de Fourier. Essa resolução é suficiente para a identificação de um número significativo de modos, revelar splittings de frequência (ver 2.7.1) e reconstruir 
perfis de linhas das pulsações.

O segundo objetivo da missão é a detecção dos planetas fora do sistema solar, baseado em medidas das mudanças na intensidade luminosa recebida devido aos trânsitos planetários. Os cientistas acreditam que poderão detectar 10 a 40 planetas de tamanho médio, similares àqueles no sistema solar. Ao mesmo tempo, o satélite está descobrindo um grande número de gigantes gasosos, aumentando significativamente a estatística atual. Até fevereiro de 2011, foram encontrados 17 exoplanetas, sendo 16 do tipo de Júpiter e 1 terrestre. Adaptando o tempo de integração e foco no canal exoplanetário, mas sem nenhuma grande alteração no desenho do experimento, variações de fluxo luminoso de até $7 \cdot 10^{-4}$ podem ser observadas pelo CoRoT em uma grande variedade de estrelas cuja magnitude varia entre 12 e 15,5. Essas variações são semelhantes ao decréscimo de luminosidade em uma estrela ocultada por um planeta ligeiramente maior que a Terra.

Programas adicionais relacionados a outras áreas da astrofísica como atividade e magnetismo estelar, variabilidade intrínseca e detecção de cometas, podem se beneficiar dos dados obtidos. Eles compreendem mais de 200.000 objetos na faixa de magnitudes de 5,5 a 16, que tem sido observados com o satélite CoRoT com tempos de amostragem de 1 segundo a 15 minutos e com precisão de 100 ppm por medida.

\subsubsection{Aspectos Gerais}

O satélite Corot foi instalado sobre a plataforma padrão Proteus do CNES (Agência Espacial Francesa), uma plataforma de órbita baixa, e foi colocado em órbita inercial polar a $896 \mathrm{~km}$ de altitude. Sua massa total é de $600 \mathrm{~kg}$, com carga útil composta de um telescópio afocal de $270 \mathrm{~mm}$, uma câmera de grande campo ( 10 ${ }^{\circ}$ de raio, no céu), equipada com quatro detectores CCD de 2048 x 2048 pixels (2 para cada programa) e eletrônica de controle, processamento e transmissão de dados. A carga útil da satélite mede 4,20 x 2,00 metros aproximadamente. Atualmente, apenas 2 detectores CCDs estão funcionando, 1 para sismologia e outro para exoplanetas.

A órbita polar permite a observação contínua de uma mesma área do céu por 150 dias sem obstrução do seu campo de visão, nem pela Terra nem pelo Sol, constituindo isso um dos grandes trunfos do experimento. Dessa forma, as observações foram divididas em dois tipos: observações longas de 150 dias de duração e observações curtas de 20 a 30 dias de 


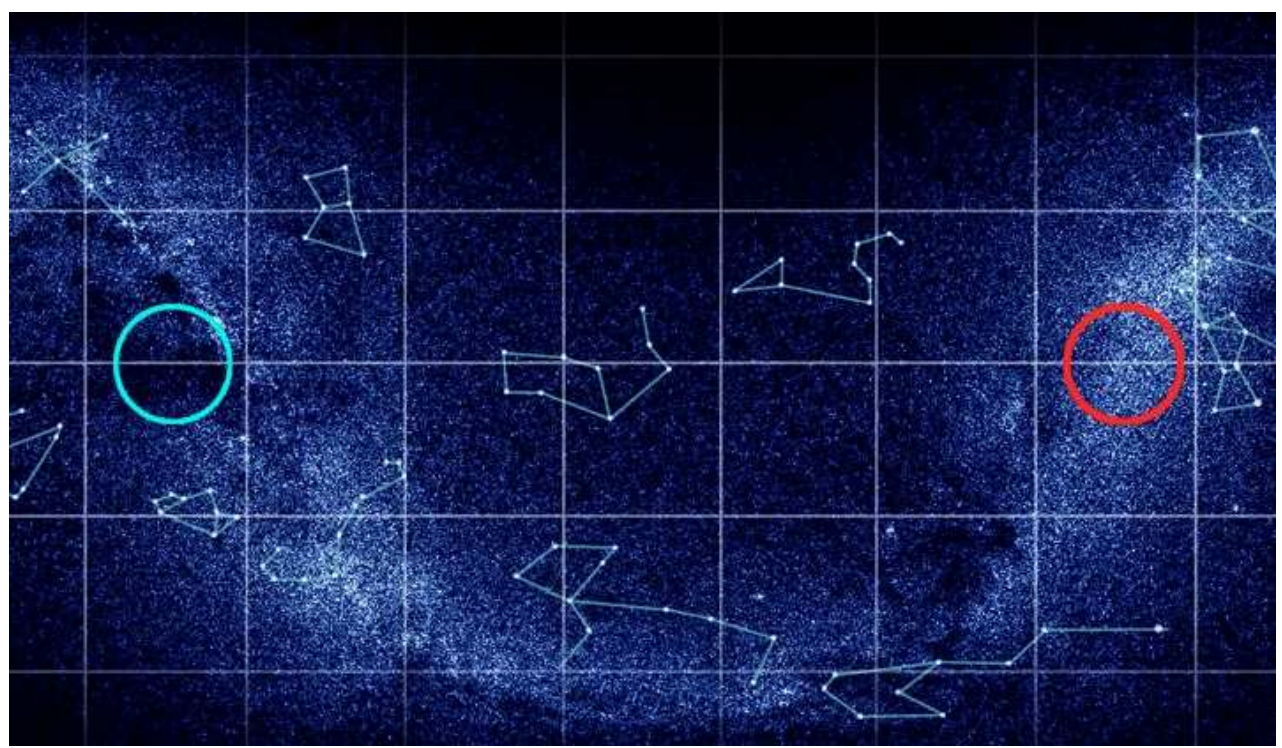

Figura 3.2: Os campos de observação da missão CoRoT. Créditos: CNES (2011).

duração, para complementar. Elas estão organizadas de modo que as observações curtas sejam realizadas entre duas observações longas do programa central. As observações longas constituem o programa central, destinado tanto a sismologia como à busca por planetas. As obervações curtas são igualmente divididas entre o programa exploratório de sismologia e programas adicionais.

A cada semestre, o satélite tem que realizar uma manobra de reversão para que o Sol não ilumine o telescópio. Isso faz com que o satélite apresente dois campos de visão no plano galáctico, um próximo ao centro da galáxia e outro ao anticentro. A Figura 3.2 mostra os dois campos de observação do CoRoT no plano galáctico.

O campo de visão é de $2,7^{\circ} \times 3,05^{\circ}$. O posicionamento esquerda/direita dos canais de sismologia e exoplanetas foi definido para o plano da órbita com o compromisso de orientar de maneira mais favorável cada canal no céu. Dentro dos critérios estabelecidos, o campo deveria apresentar pelo menos 10 alvos principais dentro dos critérios de magnitude e tipo espectral, mais de 800 estrelas secundárias com magnitude menor que 8 e uma densidade de anãs vermelhas maior que 1.500 por grau quadrado para o programa de exoplanetas.

O satélite CoRoT apresenta um período de observação maior que o satélite MOST e, portanto, uma melhor resolução. Porém, MOST apresentava um campo de visão menos restrito. 


\subsection{Kepler}

\subsubsection{Objetivo da Missão Espacial}

A missão Kepler consiste em um observatório espacial da Agência Espacial NorteAmericana (NASA) concebido para descobrir planetas parecidos com a Terra orbitando outras estrelas (e.g. Koch et al., 2010). O satélite foi colocado em órbita em março de 2009 com um tempo de vida planejado em 3,5 anos. Os detalhes da missão podem ser encontrados no site: http://kepler.nasa.gov/ .

O objetivo científico principal da missão Kepler é detectar planetas de tamanho terrestre na zona habitável de estrelas do tipo solar, determinar seu período e identificar suas características. O método escolhido é a observação de trânsitos planetários fotometricamente, o mesmo utilizado pelo satélite CoRoT. Ele fornece o período orbital do planeta e seu tamanho em relação à estrela, e, combinando isso com os parâmetros estelares e medidas de velocidade radial, é possível obter a massa, raio e densidade dos planetas. Esse método requer fotometria de alta precisão com dados de séries temporais contínuas de muitas estrelas num período de tempo grande. Apesar de ter sido projetado com o propósito explicito de detecção de planetas terrestres, os tipos de dados obtidos apresentam grande valor para a astrofísica estelar. A simologia estelar, como exemplo, também requer uma série temporal precisa e contínua. Além disso, as interpretações da ciência exoplanetária estão intimamente ligadas aos parâmetros estelares.

Para atingir estes objetivos, três requisitos fundamentais do projeto foram estabelecidos: a precisão fotométrica, o tempo de vida da missão e o número de estrelas observado. A exigência fotométrica é detectar trânsitos de planetas do tamanho da Terra $\left(R \sim 1,0 R_{\oplus}\right)$ com período de 6,5 horas (metade da duração do trânsito central para um planeta a 1 UA) de uma estrela do tipo solar com magnitude 12 e um sinal/ruído maior ou igual a quatro, quando todas as fontes de ruído estelar (variabilidade, instrumento) estão considerados (Borucki et al., 2008). Já que uma seqüência periódica de pelo menos três trânsitos é necessária, a missão deve durar três anos ou mais para detectar planetas na zona habitável. Finalmente, o campo de visão deve ser escolhido de maneira a observar um número estatisticamente significativo de estrelas do tipo solar. Assim, ele foi escolhido de modo a fornecer pelo menos 100.000 estrelas anãs. 
Além de usar os dados na busca de trânsitos planetários sequenciais e da luz refletida a partir de (não necessariamente em trânsito) planetas gigantes próximos, os dados também serão utilizados para: a obtenção de medidas de paralaxe, determinar as taxas de rotação estelar e fazer análise astrossísmica. A última análise será realizada pelo Asteroseismic Kepler Science Consortium (KASC) para medir oscilações de modos p das estrelas mais brilhantes que magnitude 11,5 no campo de visão, que têm sido observados com uma cadência de um minuto. O resultado será uma boa estimativa do raio, massa, densidade e idade das estrelas.

\subsubsection{Aspectos Gerais}

O satélite Kepler foi colocado em uma órbita heliocêntrica de perseguição da Terra em março de 2009. Sua massa é estimada em $995 \mathrm{~kg}$, com carga útil composta de um telescópio Schmidt clássico com 95 cm de diâmetro e com um campo de visão de mais de 100 graus quadrados. O fotômetro é composto de um conjunto de 42 CCDs, cada um com 50 x 25 mm e 2200 x 1024 pixels. Os CCDs são lidos a cada 3 s para evitar saturação.

Sua disposição orbital apresenta diversas vantagens em comparação com órbitas da Terra. Entre elas, o fato do satélite não passar por cinturões de radiação ou a Anomalia do Atlântico Sul, pela sombra da Terra ou aquecimento do Sol, não existir arraste atmosférico ou gradiente de gravidade agindo como torque e a variação contínua do brilho terrestre. A maior contribuição como torque nesse caso é a da luz solar. Essa órbita também proporciona que um mesmo campo de visão seja observado durante a missão. Porém, para manter o fotômetro protegido da luz solar, é necessário fazer uma rotação de $90^{\circ} \mathrm{em}$ torno do eixo óptico a cada 93 dias.

Para evitar a luz solar, o campo de visão é limitado a um campo estelar a mais de $55^{\circ}$ do plano da eclíptica. Fazendo a ánalise dos campos possíveis e contando o número de estrelas com magnitude menor que $V=14$ do catálogo USNO, mostrou-se que a região de Cygnus era a melhor opção. A região escolhida é centrada em 19h22m40s de ascensão reta e $44^{\circ} 33^{\prime}$ de declinação, logo acima do plano galáctico na direção do braço de Órion. A Figura 3.3 mostra o campo de visão do Kepler com o arranjo dos 42 detectores CCD.

O campo apresenta uma amostra de estrelas similar à da vizinhança solar e pode ser observado o tempo todo pelo satélite durante sua missão. A distância típica da estrelas 


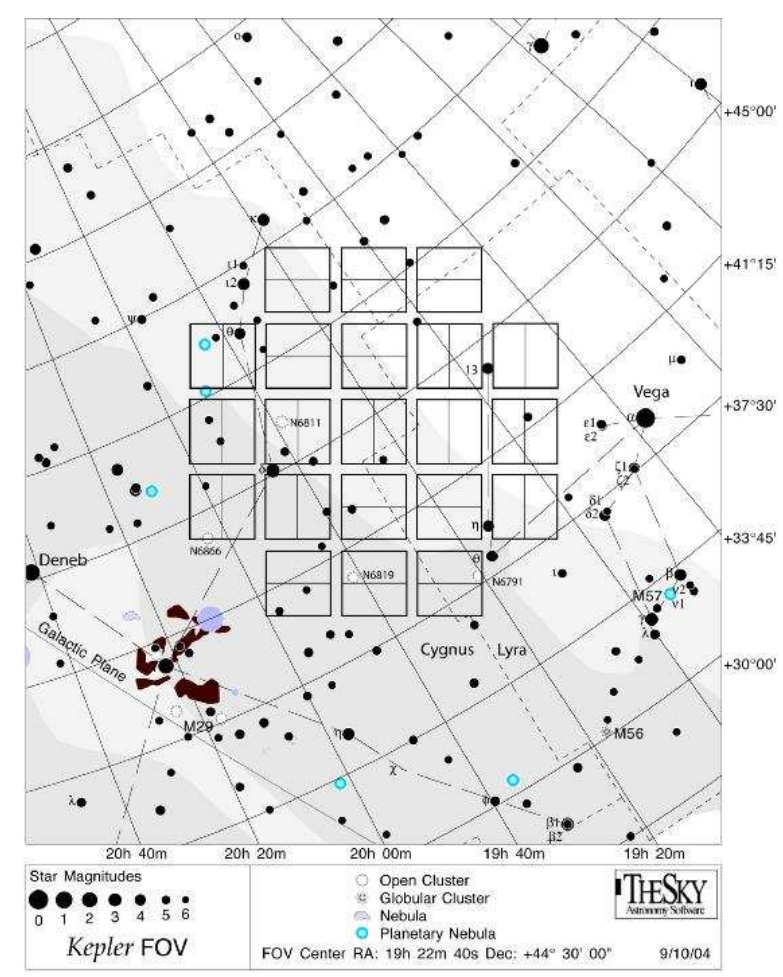

Figura 3.3: Campo de visão do satélite Kepler com o esquema dos 42 detectores CCD. Créditos: NASA (2011).

a serem utilizadas na análise vai de algumas centenas de parsecs à $1 \mathrm{kpc}$. O limite em magnitude é de cerca de $V=15-16$ para estrelas anãs do tipo F, G e K e de $V=16,5$ para as do tipo M. A orientação final do campo também minimizou o número de estrelas muito brilhantes nos CCDs. Somente cerca de uma dúzia de estrelas mais brilhantes que $V=6$ estão no campo e apenas uma, $\theta$ Cyg é mais brilhante que $V=5$.

O satélite Kepler tem um campo de visão ainda mais restrito que o do CoRoT. Porém, esse mesmo campo de visão é maior e será observado por 3,5 anos no mínimo. Isso proporciona uma maior resolução. Dentro os três satélites, Kepler apresenta o maior telescópio com 95 cm de diâmetro. 
Capítulo 4

\section{Modelos Estelares}

Neste capítulo é feita a apresentação dos modelos teóricos utilizados na análise do trabalho. Na seção 4.1, o modelo de estrutura e evolução estelar CESAM é discutido e, na seção 4.2, o modelo para o cálculo das frequências de oscilação FILOU é apresentado.

\subsection{CESAM}

O modelo CESAM é composto de um conjunto de programas e rotinas que calculam a evolução estelar em equilíbrio quasi-estático e unidimensional, incluindo rotação e difusão. Sua principal característica é a solução do equilíbrio quasi-estático pelo método collocation baseado em aproximações polinomiais projetadas em base B-spline que permite cálculos estáveis e robustos e uma restituição exata da solução (Morel, 1997). O modelo oferece também o monitoramento por apenas um parâmetro de precisão e seu melhoramento por superconvergência. Um refinamento de grade automático é implementado de modo a ajustar as localizações dos pontos de grade de acordo com as mudanças das incógnitas, cada limite entre as zonas radiativa e convectiva sendo deslocado para o ponto mais próximo da grade. No caso de modelos padrões, a evolução da composição química é resolvida por esquemas estáveis rígidos até de quarta ordem. Para modelos não-padrões, a solução das equações de difusão emprega o esquema Petrov-Galerkin com a mistura dos elementos químicos em zonas convectivas realizada por difusão turbulenta forte. Uma reconstituição precisa da atmosfera também é prevista.

O código CESAM calcula a evolução estelar desde a pré-sequência principal até o começo do ciclo $3 \alpha$ do ${ }^{4} \mathrm{He}$. Com o uso da base B-spline, o código é estruturado num "espaço numérico" onde o problema diferencial é resolvido formalmente e num "espaço 
físico" onde as equações são formadas por um método numérico qualquer usado para obter a solução, portanto, toda a física. Portanto, todos os formalismos físicos, como equação de estado (EOS), opacidades, taxas de reação nuclear, condições de contorno externas, cálculo do fluxo convectivo, são calculados em rotinas externas que podem ser fornecida pelo usuário ou tomadas da fonte sem nenhuma intervenção no "espaço numérico". Por isso, CESAM permite o cálculo de modelos estelares com várias hipóteses físicas, dados físicos, condições de contorno externas, métodos numéricos e precisão numérica, incluindo também a difusão e a perda de massa.

\subsubsection{Modelos de Equilíbrio}

Os modelos de equilíbrio são calculados a partir do formalismo de Kippenhahn e Weigert (1990) utilizando o código CESAM e são construídos incluindo efeitos da rotação em primeira ordem. Esses modelos são conhecidos como modelos de pseudo-rotação, onde a contribuição da aceleração centrífuga é levada em conta de forma esfericamente simétrica por meio da gravidade efetiva

$$
g_{\text {eff }}=g-\mathcal{A}_{c}(r)
$$

onde $g$ é a componente da gravidade local a uma distância $r$

$$
g=G \frac{m(r)}{r^{2}}
$$

com $m(r)$ a massa contida na casca esférica de raio $r, G$ a constante gravitacional. E $\mathcal{A}_{c}(r)$ corresponde à aceleração centrífuga no raio $r$

$$
\mathcal{A}_{c}(r)=\frac{2}{3} r \Omega^{2}(r)
$$

onde $\Omega$ é a velocidade de rotação. Essa contribuição esférica simétrica da rotação não muda a forma da equação de equilíbrio hidrostático. Isso não é inteiramente representativo da estrutura de uma estrela em rotação. As componentes não-esféricas da aceleração centrífuga são incluídas nos cálculos de oscilações adiabáticas na próxima seção através de análise perturbativa conforme Soufi et al. (1998).

A questão da rotação diferencial pode ser avaliada para duas hipóteses simples. A primeira considera o transporte instantâneo de momento angular por toda a estrela (conservação global) que implica assim, numa rotação uniforme. E a segunda leva em conta 


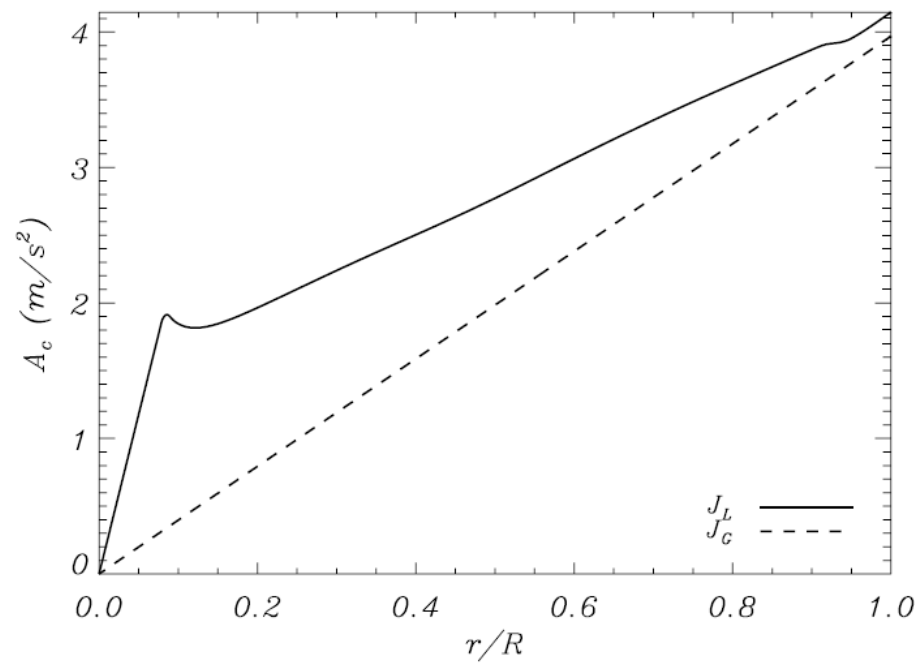

Figura 4.1: Componente radial da aceleração centrífuga para os modelos de rotação diferencial $J_{L}$ (linha contínua) e rotação uniforme $J_{G}$ (linha tracejada). As quantidades apresentadas são o caso para um modelo de $1,8 M_{\odot}$, com idade de 1,05 Gyr e raio fotométrico de $R=2,27 R_{\odot}$. Créditos: Suárez et al. (2006).

a conservação local de momento angular (rotação shellular), exceto no núcleo convectivo, onde se assume rotação de corpo rígido. Nenhuma perda de massa é considerada durante a evolução da estrela, e portanto, o momento angular total é conservado. Formalmente, a conservação de momento angular entre dois instantes $\left(t_{2}>t_{1}\right)$ pode ser expressa como

$$
\frac{2}{3} r_{1}^{2}(m) \Omega_{1}(m)=\frac{2}{3} r_{2}^{2}(m) \Omega_{2}(m)
$$

onde $m$ é a coordenada abscissa Lagrangeana. Conforme a equação 4.1, a gravidade é modificada pelo termo radial da aceleração centrífuga $\mathcal{A}_{c}$. Na Figura 4.1, é possível ver o comportamento de $\mathcal{A}_{c}$ em função do raio para as duas hipóteses consideradas (rotação uniforme e shellular) para um modelo de 1,8 $M_{\odot}, 1,05 \mathrm{Gyr}$ e raio fotométrico igual a $r=2,27 R$. A função apresenta um máximo local em $r=0,1 R$, que representa o limite do núcleo convectivo, implicando em modificações na densidade local, principalmente nessas regiões.

A equação de estado CEFF (Christensen-Dalsgaard e Daeppen, 1992) é utilizada, na qual a correção Coulombiana clássica EFF (Eggleton et al., 1973) está incluída. As tabelas de opacidade são obtidas do pacote OPAL 1996 com valores de baixa temperatura de Kurucz. As razões de abundância de elementos pesados são fixadas em valores iniciais, sendo 
indiferentes a mudanças causadas por reações nucleares e difusão. Considera-se as reações nucleares da cadeia $p p$ e do ciclo CNO. No caso dos modelos evolutivos considerados, uma blindagem eletrônica fraca é utilizada (Suárez et al., 2009). No caso de valores de temperatura acima de $T \geq 7 \cdot 10^{7} \mathrm{~K} \simeq 7 \mathrm{keV}$, o plasma é completamente ionizado e por isso a opacidade média de Rosseland é reduzida ao espalhamento Compton por elétrons livres (Cox e Giuli, 1968).

Em cada camada, de acordo com uma escolha feita a priori, os critérios de Schwarzchild ou de Ledoux permitem decidir se o transporte de energia é feito por radiação ou convecção (Morel, 1997). A convecção é tratada, utilizando-se o formalismo padrão de comprimento de mistura (ver Böhm-Vitense, 1958). Além disso, no limite entre zonas radiativas e convectivas, a velocidade dos redemoinhos convectivos é diferente de zero e, portanto, os movimentos convectivos podem penetrar na zona radiativa. Devido à pequena escala de tempo característica da convecção, a matéria é homogeneizada nas zonas de overshoot/undershoot. Os parâmetros convectivos de eficiência e overshooting do núcleo usados são $\alpha_{M L}=l_{m} / H_{p}=1,8$ e $d_{o v}=l_{o v} / H_{p}=0,2$, respectivamente, onde $l_{M L}$ é o livre caminho médio dos elementos convectivos, $H_{p}$ é a escala de altura da pressão e $l_{o v}$ é a distância de penetração dos elementos convectivos.

O programa começa pelo cálculo dos modelos de equilíbrio na sequência principal de idade zero (ZAMS) e continua até o final da fase de sequência principal. A massa inicial, a velocidade superficial e a metalicidade definida por meio das abundâncias de $\mathrm{H}$ e He, são parâmetros de entrada para o cálculo dos modelos.

\subsection{FILOU}

As autofrequências adiabáticas dos modelos de equilíbrio são calculadas com o código FILOU (e.g. Suárez e Goupil, 2008) que emprega o método perturbativo até segunda ordem (força centrífuga e força de Coriolis) em seu cálculo. Este código utiliza como modelos de entrada, os modelos de equilíbrio calculados com CESAM. FILOU foi originalmente desenvolvido por Minh e Léon (1995) no Observatório de Paris-Meudon é passou por diversas modificações e melhorias a fim de introduzir correções devido ao efeito de rotação nas frequências de oscilação. A inclusão dessas correções (até segunda ordem e incluindo efeitos de degenerescência próxima) foram feitas por Suárez (2002). Nesse trabalho, o 
autor estendeu o cálculo das oscilações para modelos com perfil de rotação diferencial da forma

$$
\Omega(r)=\bar{\Omega}\left(1+\eta_{0}(r)\right),
$$

onde $\bar{\Omega}$ representa a velocidade angular de rotação na superfície e $\eta_{0}(r)$ é a função radial, sendo esse tipo de rotação conhecido como rotação shellular. A extensão para o cálculo de rotação shellular é a característica mais marcante do código, tornando-o único.

Nesse caso, as oscilações são calculadas a partir dos modelos de pseudo-rotação que contêm a contribuição simétrica esférica da aceleração centrífuga. Já os efeitos de componentes não-esféricas da deformação da estrela são incluídos através de uma perturbação nas equações de oscilação. Um exemplo disso é a perturbação da densidade média $\rho_{0}$ de um modelo de pseudo-rotação, considerada na forma $\rho_{2}=p_{22}(r) P_{2}(\cos \theta)$, onde $p_{22}(r)$ é definido por Suárez et al. (2006).

Além disso, quando se considera a degenerescência próxima, as autofrequências e as autofunções de modos próximos podem ser escritos como

$$
\omega^{d}=\tilde{\omega}_{0}+\tilde{\omega}_{1}+\tilde{\omega}_{2}
$$

e

$$
\xi=\sum_{j=a, b} \alpha_{j}\left(\xi_{0, j}+\xi_{1, j}\right)
$$

onde $\tilde{\omega}_{0}\left(\tilde{\omega}_{0, a}+\tilde{\omega}_{0, b}\right) / 2$ e $\alpha_{j}$ representa os coeficientes de combinação linear entre dois modos considerados degenerados e os índices $a$ e $b$ representam os modos rotacionalmente acoplados. As correções de primeira e de segunda ordem são representadas por $\tilde{\omega}_{1}$ e $\tilde{\omega}_{2}$, respectivamente; $\tilde{\omega}_{1}$ e $\tilde{\omega}_{2}$ representam as correções de primeira e segunda ordem na autofrequência, respectivamente, e $\xi_{0, j}$ e $\xi_{1, j}$, as autofunções não-perturbada e perturbada em primeira ordem. 


\subsubsection{Frequências de Oscilação de um Modelo de Pseudo-Rotação}

FILOU resolve o sistema de autovalores gerado devido a perturbação e linearização das equações de hidrodinâmicas para um modelo de pseudo-rotação definido por

$$
\begin{aligned}
x \frac{d y_{01}}{d x} & =\lambda-3 y_{01}+\frac{\Lambda}{C_{r} \sigma_{0}^{2}} y_{02} \\
x \frac{d y_{02}}{d x} & =\left(C_{r} \sigma_{0}^{2}-A^{*}\right) y_{01}+\left(A^{*}+1-U_{\chi}\right) y_{02}-A^{*} y_{03} \\
x \frac{d y_{03}}{d x} & =\left(1-U_{\chi}\right) y_{03}+y_{04} \\
x \frac{d y_{04}}{d x} & =\frac{U}{1-\sigma_{r}}\left[A^{*} y_{01}+V_{g}\left(y_{02}-y_{03}\right)\right]+\Lambda y_{03}-U_{\chi} y_{04}
\end{aligned}
$$

onde $x=r / R, R$ é o raio estelar e as quantidades adimensionais usadas são

$$
y_{01}=\frac{\xi_{r}}{r}, \quad y_{02}=\frac{1}{g_{e f f} r}\left(\phi^{\prime}+\frac{p^{\prime}}{\rho}\right), \quad y_{03}=\frac{\phi^{\prime}}{g_{e f f} r}, \quad y_{04}=\frac{1}{g_{e f f}} \frac{d \phi^{\prime}}{d r},
$$

onde $g_{\text {eff }}$ é a gravidade efetiva e $p^{\prime}$ e $\phi^{\prime}$ são a pertubação euleriana da pressão e do potencial gravitacional, respectivamente. A frequência é expressa em termos da frequência adimencional ao quadrado

$$
\sigma_{0}^{2}=\frac{\omega^{2}}{G M / R^{3}}
$$

assim como as quantidades adiabática seguem a notação clássica de Unno et al. (1989)

$$
\begin{aligned}
A^{*} & =\frac{1}{\Gamma} \frac{d \ln p}{d \ln r}-\frac{d \ln \rho}{d \ln r}, \\
V & =-\frac{d \ln p}{d \ln r}, \quad V_{g}=\frac{V}{\Gamma_{1}}, \\
U & =\frac{d \ln M_{r}}{d \ln r} .
\end{aligned}
$$

Como definido em Soufi et al. (1998), as seguintes variáveis também são empregadas

$$
\begin{aligned}
C & =\left(\frac{r}{R}\right)^{2} \frac{M}{M_{r}}, \quad C_{r}=\frac{C}{1-\sigma_{r}}, \quad \sigma_{r}=\frac{\mathcal{A}_{c}}{g}, \\
\chi & =\frac{\mathcal{A}_{c}}{g_{\text {eff }}}\left(U-3+\frac{d \Omega^{2} / \bar{\Omega}^{2}}{d r}\right), \quad U_{\chi}=U+\chi, \\
\lambda & =V_{g}\left(y_{01}-y_{02}+y_{03}\right), \quad \Lambda=l(l+1),
\end{aligned}
$$

onde $M$ e $M_{r}$ são a massa estelar e a massa contida na esfera de raio r, respectivamente. 


\subsubsection{Condições de Contorno}

O sistema definido em 4.8 é resolvido pelo código para as seguintes condições de contorno

$$
\begin{aligned}
y_{02}+y_{01} \frac{3}{V_{g}}=0, \quad 3 y_{01}+y_{04}=0 & (l=0) \\
y_{01}-y_{02} \frac{l}{C_{r} \sigma_{0}^{2}}=0, \quad y_{04}-l y_{03}=0 & (l \neq 0)
\end{aligned}
$$

no centro da estrela e,

$$
\begin{gathered}
y_{01}=1, \\
y_{04}+(l+1) y_{03}=0 \\
y_{01}\left(1+\frac{\Lambda}{V C \sigma_{0}^{2}}-\frac{4+C \sigma_{0}^{2}}{V}\right)-y_{02} \quad\left(1-\frac{\Lambda}{V C \sigma_{0}^{2}}\right)+y_{03}\left(1+\frac{l+1}{V}\right)=0
\end{gathered}
$$

na superfície da estrela. O modelo considera as condições de contorno para oscilaçõea adiabáticas descritas na seção 2.4.

A solução do sistema com essas condições de contorno é a frequência de ordem zero $\omega_{0}$ que inclui efeitos esféricos simétricos da perturbação de pressão, densidade e potencial gravitacional.

\subsubsection{Autofunções Perturbadas em Primeira Ordem}

Quando efeitos de degenerescência próxima são considerados, correções de primeira ordem nas autofunções são necessárias. Considera-se as variáveis admensionais da equação 4.9 com as quantidades perturbadas em primeira ordem $\left(\xi_{1, r}, \phi_{1}^{\prime}, p_{1}^{\prime}\right)$, e as soluções de ordem zero obtidas da equação 4.8. O sistema perturbado em primeira ordem fica

$$
\begin{aligned}
x \frac{d y_{1}}{d x} & =\lambda_{1}-3 y_{1}+\frac{\Lambda}{C_{r} \sigma_{0}^{2}} y_{2}+\left(y_{01}+z_{0}\right)\left(1+\eta_{0}\right)-\left(\eta_{0}+\sigma_{1}\right) \Lambda z_{0}, \\
x \frac{d y_{02}}{d x} & =\left(C_{r} \sigma_{0}^{2}-A^{*}\right) y_{1}+\left(A^{*}+1-U_{\chi}\right) y_{2}-A^{*} y_{3}+\left(\sigma_{1}+\eta_{0}\right) y_{01}-\left(1+\eta_{0}\right) z_{0}, \\
x \frac{d y_{3}}{d x} & =\left(1-U_{\chi}\right) y_{3}+y_{4}, \\
x \frac{d y_{4}}{d x} & =\frac{U}{1-\sigma_{r}}\left[A^{*} y_{1}+V_{g} y_{2}-V_{g} y_{3}\right]+\Lambda y_{3}-U_{\chi} y_{4},
\end{aligned}
$$

onde $\lambda_{1}=V_{g}\left(y_{1}-y_{2}+y_{3}\right)$ e $z_{0}=y_{02} / C \sigma_{0}^{2}$. A componente horizontal de $\xi_{1}$ pode ser escrita como

$$
z_{1}=\frac{y_{2}}{C \sigma_{0}^{2}}+\frac{1+\eta_{0}}{\Lambda} y_{01}+\left(\frac{1+\eta_{0}}{\Lambda}-\sigma_{1}\right) z_{0}
$$


onde $\sigma_{1}=C_{L}-J_{0}$ representa a correção de primeira ordem da autofrequência correspondente.

\subsubsection{Correções de Primeira e Segunda Ordem}

As correções de segunda ordem computadas por FILOU na presença de degenerescência próxima são

$$
\tilde{\omega}_{2}=\left(\frac{\mu_{b}+\mu_{a}}{2}\right) \pm \sqrt{\mathcal{H}_{2, a b}^{(2)}}
$$

no caso que $\delta \omega_{0}=\omega_{0, a}-\omega_{0, b}$ é $O\left(\Omega^{2}\right)$, e

$$
\tilde{\omega}_{2}=\left(\frac{v_{b}+v_{a}}{2}+\frac{\mu_{b}+\mu_{a}}{2}+\frac{\delta \omega_{0}^{2}}{8 \tilde{\omega}_{0}}\right) \pm \sqrt{\mathcal{H}_{2, a b}^{(1)}}
$$

se $\delta \omega_{0}$ é $O(\Omega)$. Em ambos os casos, os efeitos de degenerescência própria estão implicitos, os quais são calculados a partir de

$$
\tilde{\omega}_{1}=\frac{\omega_{1, a}+\omega_{1, b}}{2} \pm \sqrt{\mathcal{H}_{1, a b}}
$$

Os termos são definidos em Suárez et al. (2006). As variáveis $v$ e $\mu$ contêm as correções por efeito de rotação nas autofrequências individuais obtidas pelo sistema 4.8. As correções são incorporadas por FILOU segundo a notação de Saio,

$$
\omega_{j, 2}=\omega_{0, j}+\bar{\Omega}\left(C_{L}-1-J_{0}\right)+\frac{\bar{\Omega}^{2}}{\omega_{0, j}}\left(D_{0}+m^{2} D_{1}\right),
$$

onde $D_{0} \equiv X_{1}+X_{2}$ e $D_{1} \equiv Y_{1}+Y_{2}$. As definições detalhadas se encontram em (Suárez et al., 2006, Eqs. 8-15). 
Capítulo 5

\section{Sismologia e Rotação ao Longo da Sequência Principal}

O objetivo principal desse capítulo é mostrar e discutir os resultados de modelos numéricos de estrelas na sequência principal calculados para velocidades de rotação de 20 - $100 \mathrm{~km} / \mathrm{s}$. Na seção 5.1, mostra-se as características dos modelos calculados. Os diagramas Hertzsprung-Russel dos modelos são apresentados na seção 5.2. Na seção 5.3, os splittings rotacionais dos modos $\mathrm{l}=0, \mathrm{n}=1$ e $\mathrm{l}=1, \mathrm{n}=-2,-1,1,2$ são apresentados. Já na seção 5.4, as assimetrias desses mesmos modos são apresentadas. Por último, na seção 5.5, modelos representativos da estrela HD50844 são comparados a dados observacionais.

\subsection{Características dos Modelos}

Para investigar os efeitos da rotação nas propriedades das estrelas da sequência principal, modelos estelares de massas intermediárias, compreendendo 2 a $8 M_{\odot}$, foram gerados utilizando o procedimento discutido na seção 4.1. O cálculo considera modelos de pseudorotação com velocidades superficial de 20 - $100 \mathrm{~km} / \mathrm{s}$. Uma metalicidade solar $(\mathrm{X}=0,7$ e $\mathrm{Y}=0,28)$ foi assumida. O transporte convectivo é descrito pela teoria MLT, com o parâmetro $\alpha_{M L}=1,8$ (valor solar) e o parâmetro de overshooting $d_{o v}=0,2$. O último parâmetro é fixado de acordo com Schaller et al. (1992) para massas intermediárias. A Tabela 5.1 apresenta o súmario dos parâmetros físicos utilizados para gerar os modelos de equilíbrio, ou seja, modelos na ZAMS.

A trajetória evolutiva escolhida para análise se extende da ZAMS até o ponto onde a temperatura efetiva se torna mínima na sequência principal. Isso acontece antes da contração do núcleo. Esse intervalo de evolução foi escolhido pois na faixa de massa utilizada, este é o intervalo em que garante-se homogeneidade de configuração dos modelos. 
Tabela 5.1 - Parâmetros físicos utilizados nos modelos de equilíbrio.

\begin{tabular}{cc}
\hline \hline massa & $2-8\left(M_{\odot}\right)$ \\
\hline velocidade superficial de rotação & $20-100 \mathrm{~km} / \mathrm{s}$ \\
\hline metalicidade & $\mathrm{X}=0,7$ \\
& $\mathrm{Y}=0,28$ \\
\hline parâmetro de comprimento de mistura & $\alpha_{M L}=1,8$ \\
\hline parâmetro de overshooting & $d_{o v}=0,2$ \\
\hline \hline
\end{tabular}

O diagrama Hertzsprung-Russel (diagrama HR), contendo as trajetórias evolutivas dos modelos calculados com velocidade superficial de $20 \mathrm{~km} / \mathrm{s}$ podem ser visualizados na Figura 5.1. Os incrementos de $\log L$ se tornam maiores para valores maiores de $M$, enquanto as mudanças de $\log T_{\text {eff }}$ são menos expressivas. Nessa fase evolutiva, as estrelas produzem energia através da fusão nuclear do hidrogênio em hélio em seu núcleo. No caso geral das massas intermediárias, as trajetórias evolutivas são similares, todos eles apontando para uma direção acima da ZAMS. Esse é o caso para um evolução produzindo modelos quimicamentes não homogêneos (composto de um núcleo de He e um envelope rico de H). Isso significa que as estrelas desenvolvem inomogeneidades químicas em seu interior.

As estrelas nessa faixa de massas apresentam uma temperatura central elevada, de modo que a produção de energia ocorre através do ciclo CNO. A maior sensibilidade com a temperatura do ciclo CNO comparado com o ciclo p-p faz com que a produção de energia seja mais concentrada no centro do que para massas menores. No entanto, isso requer um gradiente de temperatura maior para transportar a energia para as partes mais externas da estrela. O gradiente radiativo é maior que o adiabático e as regiões internas se tornam então instáveis, estabelecendo um núcleo convectivo (Kippenhahn e Weigert, 1990). Já nas camadas externas, o gradiente radiativo é menor que o adiabático e o transporte de energia ocorre por radiação. Assim, uma estrela de massa intermediária na sequência principal é composta por um núcleo convectivo cercado por um envelope em equilíbrio radiativo.

A presença de um núcleo convectivo assegura que as regiões internas da estrela sejam bem misturadas. O hélio é produzido por queima do hidrogênio e misturado por todo o núcleo. Deste modo, não existe um gradiente de abundância de hélio do centro para a periferia da região central. Em vez disso, o núcleo convectivo inteiro está disponível como fonte de combustível para produção de energia no centro da estrela. Por esta razão, a 


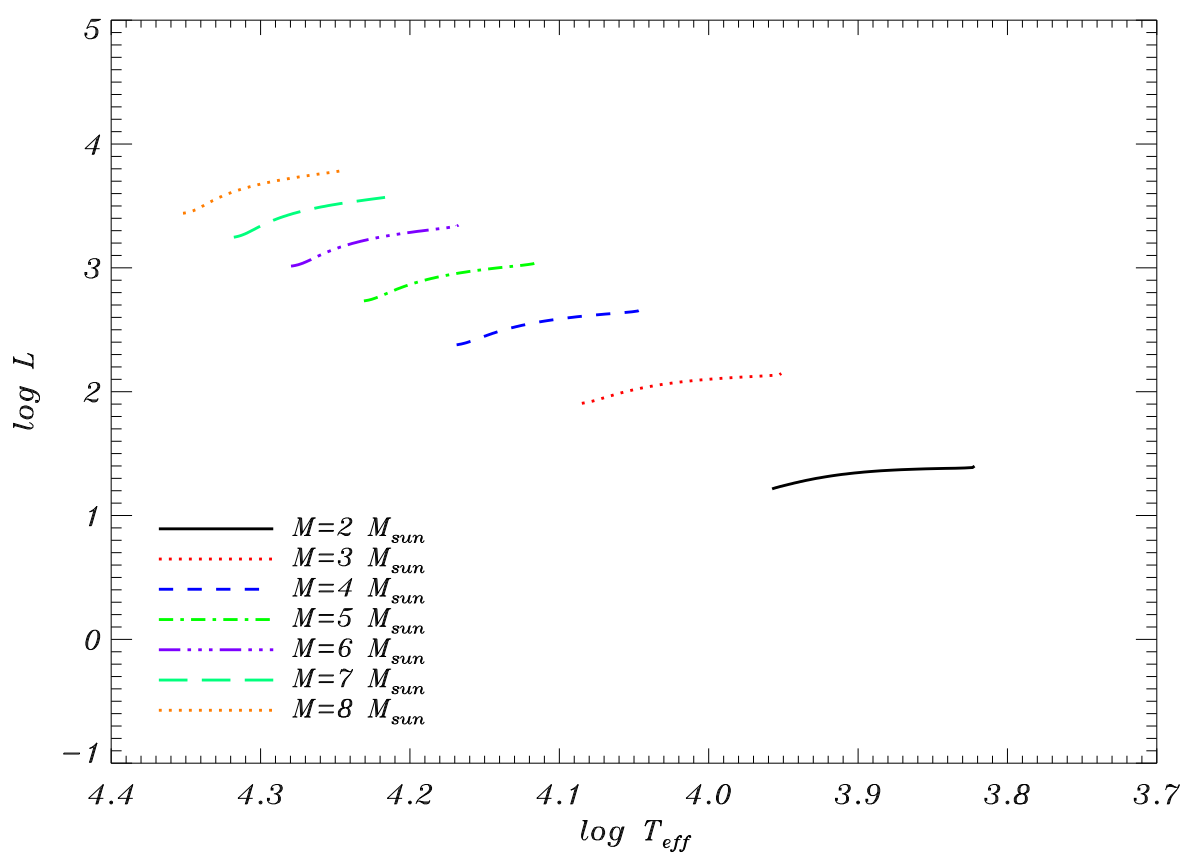

Figura 5.1: Diagrama HR contendo as trajetórias evolutivas para os modelos calculados com velocidade superficial de $20 \mathrm{~km} / \mathrm{s}$.

produção de energia é notavelmente constante nestas estrelas até que o núcleo convectivo esteja praticamente esgotado de hidrogênio (Padmanabhan, 2001). Mesmo quando se aproxima do esgotamento, com o ciclo CNO depende fortemente da temperatura, os déficites produzidos pelo declínio da quantidade de hidrogênio disponível, podem ser compensados por pequenos aumentos na temperatura e poucas alterações na estrutura da estrela. Na verdade, até próximo de 99 por cento da massa do núcleo convectivo ser convertido em hélio, mudanças realmente significativas ocorrem na estrutura da estrela.

\subsection{Efeitos da Rotação no Diagrama Hertzsprung-Russel}

A rotação introduz diversas modificações na estrutura da estrela. Como uma estrela não pode ser considerada como um corpo rígido, a rotação distorce-a e cria uma configuração oblata. Esse é o principal efeito que pode ser visualizado. Os desvios na esfericidade e as mudanças na luminosidade são de fundamental importância para os efeitos observáveis da rotação na radiação emitida pela estrela. A influência da rotação nos parâmetros físicos básicos dos modelos podem ser visualizados nas Figuras 5.2 e 5.3. 


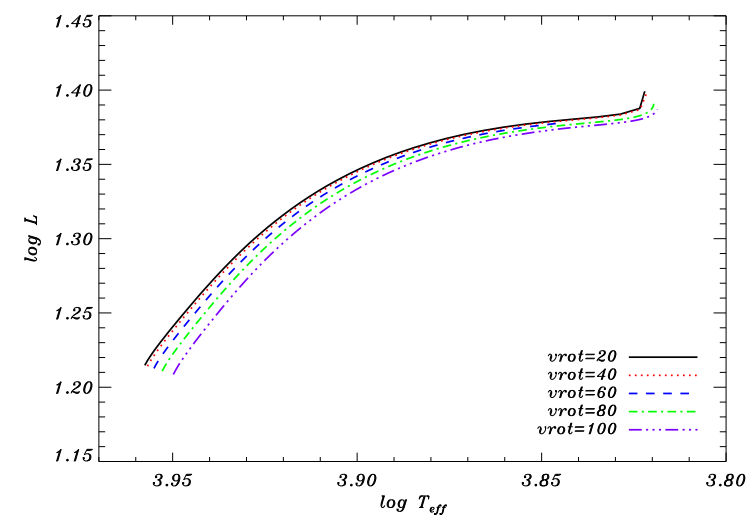

(a) $\mathrm{M}=2 M_{\odot}$

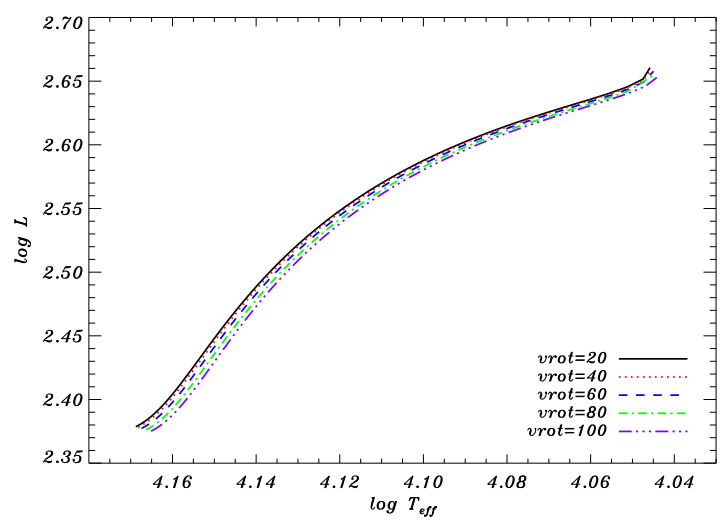

(c) $\mathrm{M}=4 M_{\odot}$

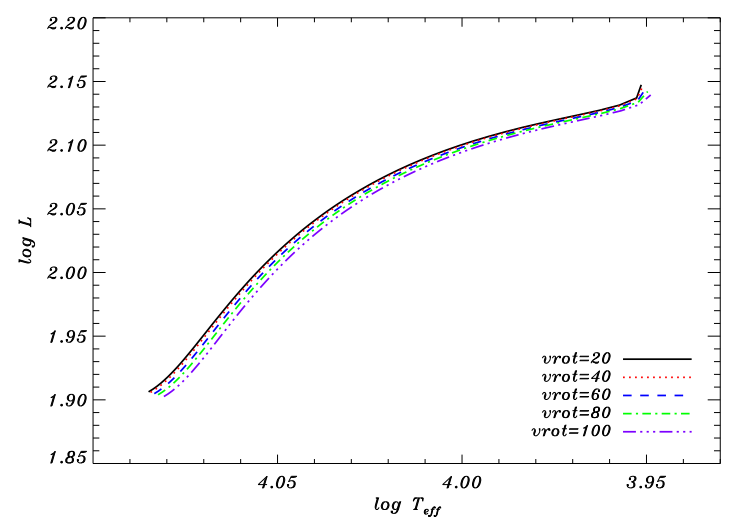

(b) $\mathrm{M}=3 M_{\odot}$

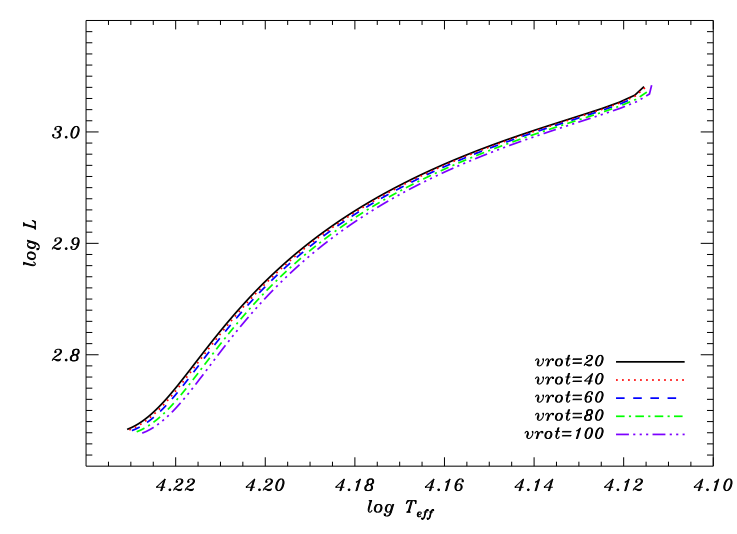

(d) $\mathrm{M}=5 M_{\odot}$

Figura 5.2: Diagrama HR contendo as trajetórias evolutivas para as massas a) $2 M_{\odot}$, b) $3 M_{\odot}$, c) $4 M_{\odot}$ e d) $5 M_{\odot}$ mostrando a influência da rotação com velocidade superficial de 20, 40, 60, 80 e $100 \mathrm{~km} / \mathrm{s}$. 


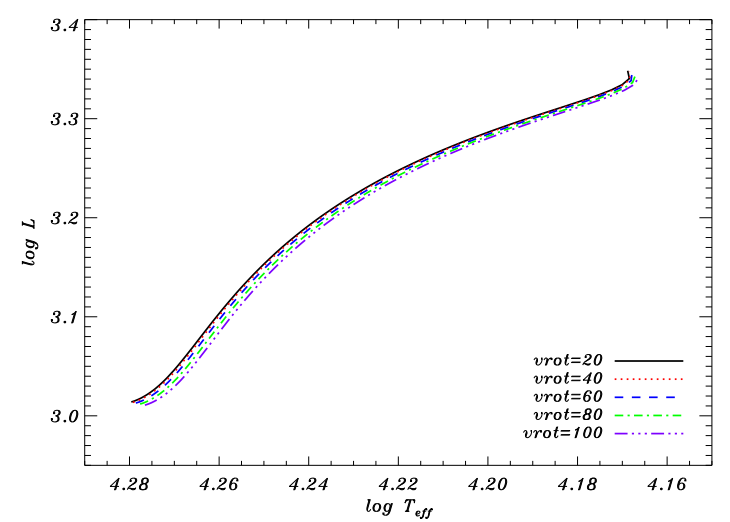

(a) $\mathrm{M}=6 M_{\odot}$

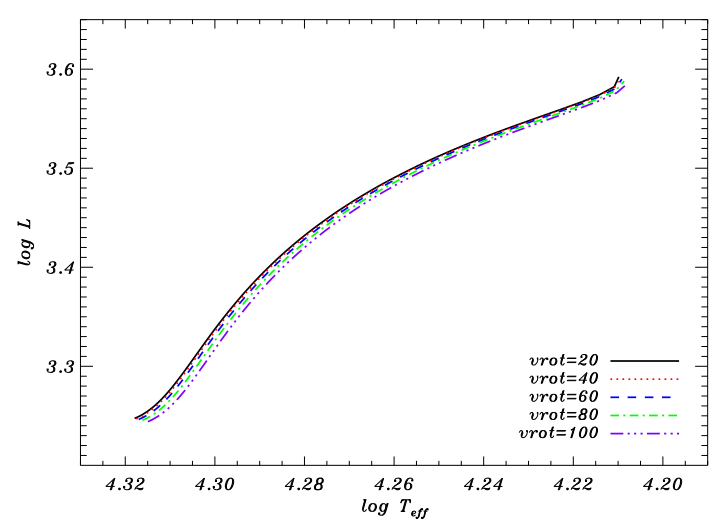

(b) $\mathrm{M}=7 M_{\odot}$

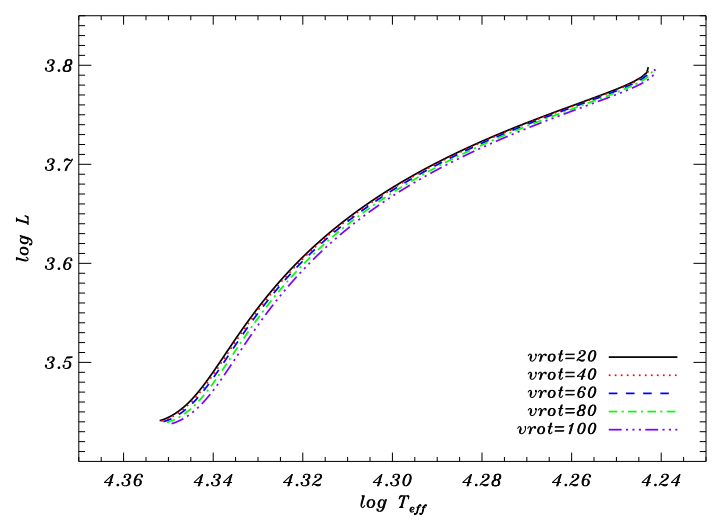

(c) $\mathrm{M}=8 M_{\odot}$

Figura 5.3: Mesmo da Figura 5.2 para as massas a) $6 M_{\odot}$, b) $7 M_{\odot}$ e c) $8 M_{\odot}$. 
Considerando um modelo baritrópico com um envelope radiativo, esse apresenta um fluxo emergente $|\overrightarrow{\mathcal{F}}|$ que varia proporcionalmente com a gravidade efetiva superficial $g$ de acordo com a Lei de von Zeipel de escurecimento por gravidade (Tassoul, 2000). Como essa quantidade é menor nos pólos que no equador, a temperatura efetiva e o brilho superficial são, portanto, menores no equador do que no pólo. Isso implica que a magnitude e os índices de cor de uma estrela com rotação serão funções do ângulo entre a linha de visada e o eixo de rotação. Uma estrela pole-on (vista a partir do pólo) parece mais brilhante que uma estrela sem rotação de mesma massa, mas com cor praticamente igual. Isso acontece porque a linha de visada está direcionada de modo a observar a região polar brilhante assim como a grande área projetada resultante do formato oblato da estrela. Uma estrela vista equator-on (vista a partir do equador) por outro lado, apresenta um brilho reduzido e é mais avermelhada que outro modelo sem rotação de mesma massa, ou seja, o escurecimento de limbo reduz o brilho da região polar enquanto que o escurecimento por gravidade torna o equador mais frio. Isso pode ser visualizado nos diagramas HRs apresentados. No caso, os modelos são considerados equator-on.

Nos diagramas HR dos modelos calculados, observa-se que para massas baixas, o efeito da rotação é maior. Isso ocorre devido a escolha da velocidade de rotação superficial e não a velocidade angular. Como uma estrela de massa maior apresenta um raio maior, sua velocidade angular será maior do que uma de baixa massa para uma mesmo velocidade de rotação superficial.

\subsection{Análise do Splitting Rotacional}

Para entender melhor como a rotação afeta a estrutura interna dos modelos, calculamos as frequências de oscilação dos modelos de equilíbrio seguindo o procedimento apresentado na seção 4.2. Esse método pode ser utilizado para rotatores lentos/moderados $\left(v_{r o t, s} \leq 120 \mathrm{~km} / \mathrm{s}\right)$ (Suárez et al., 2006). Para maiores velocidades, os efeitos da aceleração centrífuga são muito grandes e o método perturbativo não é mais válido.

Nos limites de validade do método, o estudo foi restringido a estrelas com velocidades moderadas (Tabela 5.1). Os modos de baixa ordem e $1=1$ foram escolhidos para serem analisados. A Figura 5.4 apresenta os comportamentos calculados da frequência central $(\mathrm{m}=0)$ em função da temperatura efetiva para os modos $g_{2}, g_{1}, p_{1}$ e $p_{2}$. 


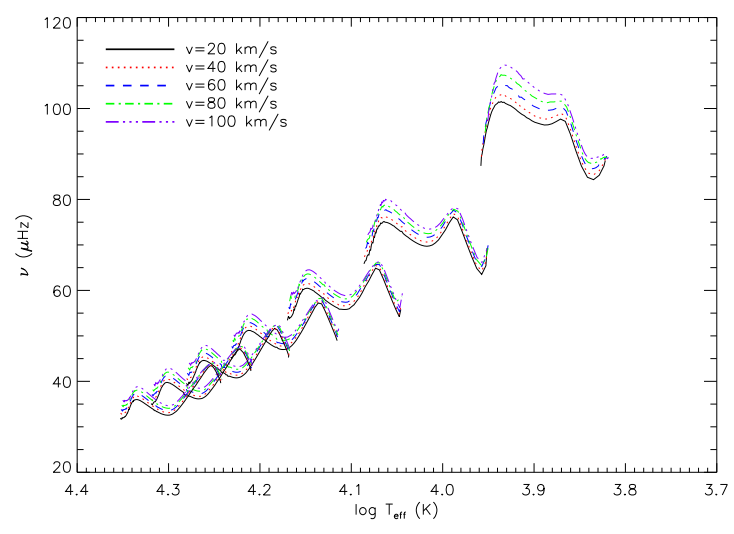

(a) modo g2

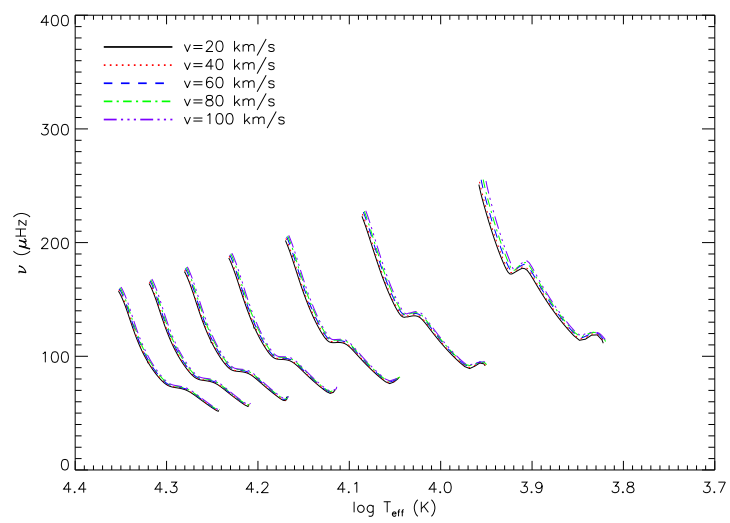

(c) modo p1

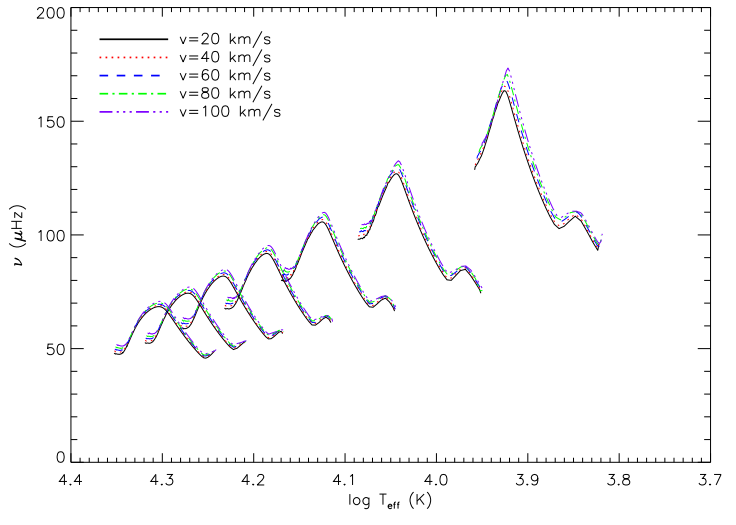

(b) modo g1

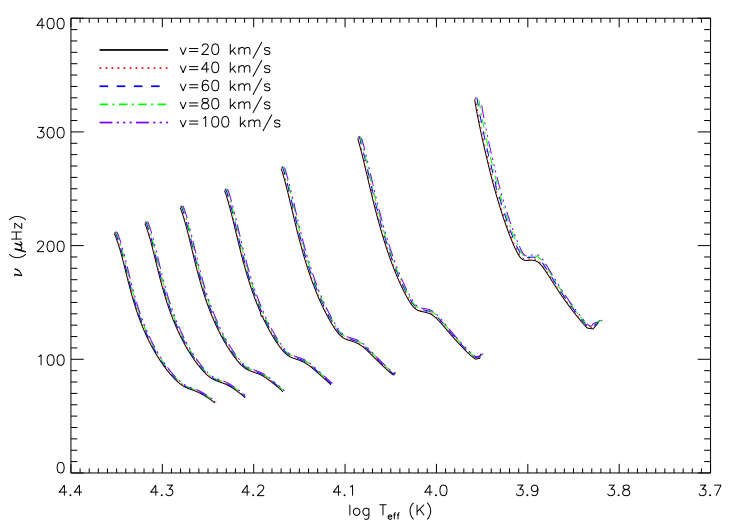

(d) modo p2

Figura 5.4: Comportamento da frequência central $(\mathrm{m}=0)$ dos modos a) $\left.\left.g_{2}, \mathrm{~b}\right) g_{1}, \mathrm{c}\right) p_{1}$ e d) $p_{2}$ em função da temperatura efetiva para as trajetórias evolutivas das massas 2 a $8 M_{\odot}$ (direita para a esquerda) mostrando a influência da rotação com velocidade superficial de 20, 40, 60, 80 e $100 \mathrm{~km} / \mathrm{s}$. 
Em termos gerais, o comportamento dos modos é similar para toda a faixa de massas considerada nos modelos. Porém, quanto maior a massa, os pontos de inversão ao longo da evolução da autofrequência se tornam mais suaves. No caso do modo $g_{2}$, durante a evolução do modelo calculado, a frequência calculada apresenta dois máximos. Porém, no caso de modelos de estrelas menos massivas, a frequência máxima no começo da evolução é maior. O contrário ocorre quando a massa dos modelos aumenta. O modo $g_{1}$ também apresenta um máximo durante a evolução na sequência principal. Já os modos p possuem autofrequências decrescentes ao longo da evolução.

O comportamento das frequências não é monotônico nem mesmo na região da sequência principal que está sendo considerada, onde as alterações externas da estrela são suaves. Isso é um primeiro indício de como as frequências observadas na superfície são indicadores de grandezas definidoras da estrutura interna da estrela. A diferença entre os modelos de alta massa e baixa massa para o modo $g_{2}$ indica diferenças na estrutura interna dos modelos.

A influência da rotação não é expressiva na frequência central dos tripletos. Para melhor caracterizar os efeitos da rotação, analisou-se os splittings rotacionais dos modos considerados. Segundo a seção 2.7.1, o splitting rotacional é definido como

$$
S_{j}=\frac{1}{2}\left(\omega_{+1, j}-\omega_{-1, j}\right),
$$

onde $\omega_{m, j}$ são as frequências $\mathrm{m}=-1,+1$ do modo $\mathrm{j}$.

O comportamento do splitting ao longo da evolução pode ser visto nas Figuras 5.5 a 5.12 que correspondem aos modelos calculados para os modos $g_{2}, g_{1}, p_{1}$ e $p_{2}$ e para a faixa de massa analisada.

O efeito óbvio da rotação pode ser visualizado. Quanto maior a rotação, maior é o splitting para todos modelos computados. Como se pode observar das Figuras, o splitting em geral decresce com a evolução da estrela, já que ao longo da sequência principal, a estrela expande, diminuido a sua rotação conservando momento angular. No entanto, este apresenta alguns máximos e mínimos indicando que o splitting apresenta também dependência com outros parâmetros físicos como mostra a equação 2.142.

O comportamento é similar para todas as massas. A Figura 5.13 mostra o comportamento do splitting rotacional para modelos na faixa de massas analisada com uma veloci- 


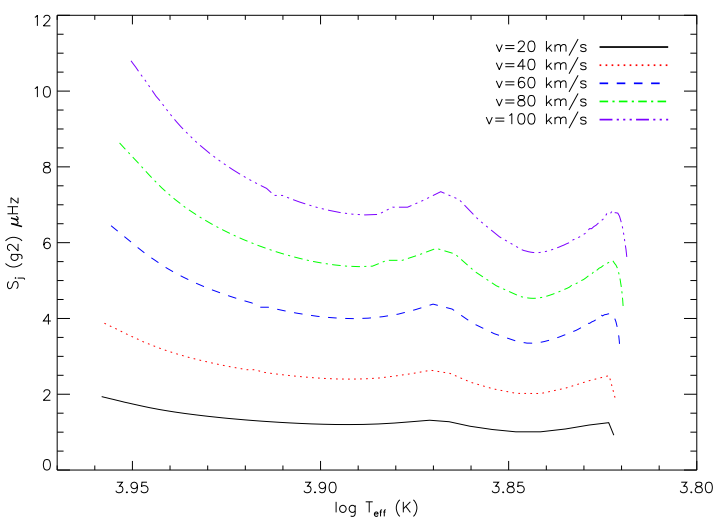

(a) $\mathrm{M}=2 M_{\odot}$

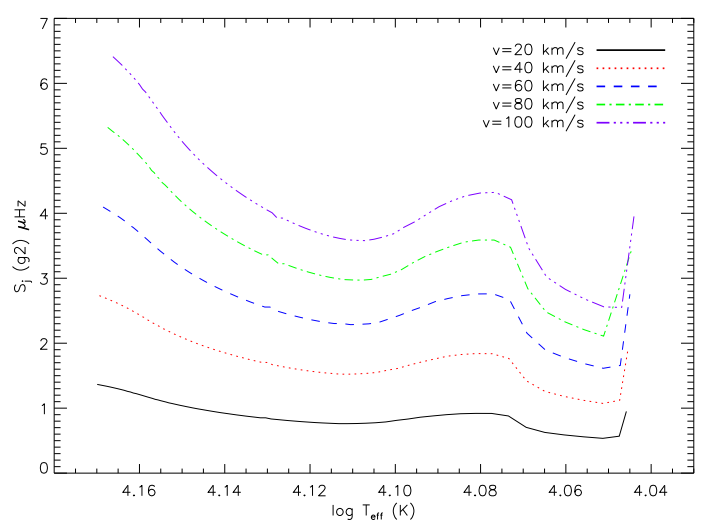

(c) $\mathrm{M}=4 M_{\odot}$

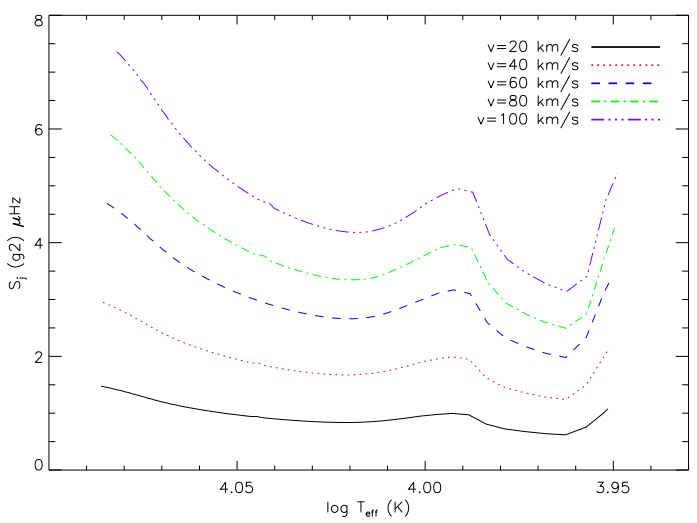

(b) $\mathrm{M}=3 M_{\odot}$

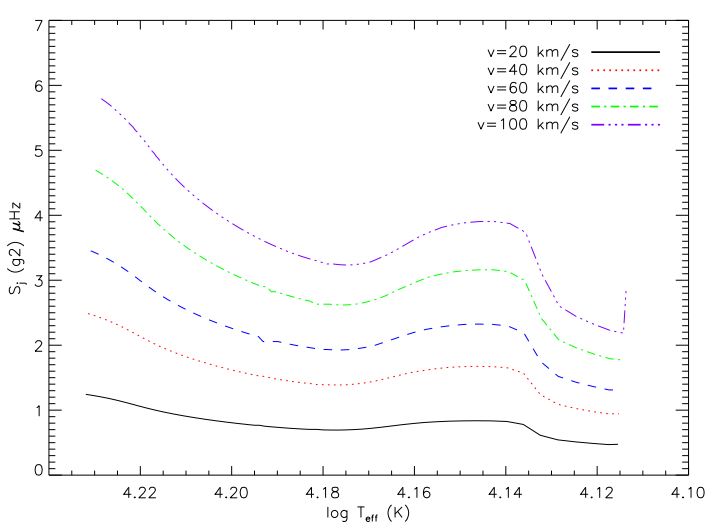

(d) $\mathrm{M}=5 M_{\odot}$

Figura 5.5: Comportamento do splitting rotacional do modo $g_{2}$ em função da temperatura efetiva para as trajetórias evolutivas das massas a) $2 M_{\odot}$, b) $3 M_{\odot}$, c) $4 M_{\odot}$ e d) $5 M_{\odot}$ mostrando a influência da rotação com velocidade superficial de 20, 40, 60, 80 e $100 \mathrm{~km} / \mathrm{s}$. Existe um problema de convergência dos códigos utilizados nos pontos à direita (picos) das Figuras b), c) e d). 


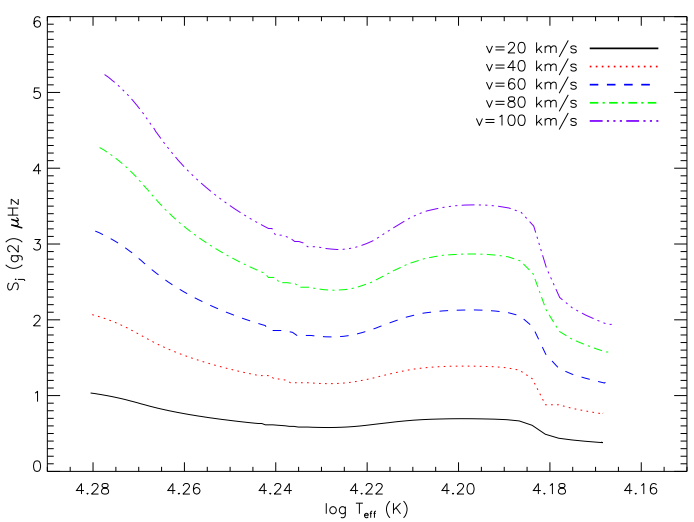

(a) $\mathrm{M}=6 M_{\odot}$

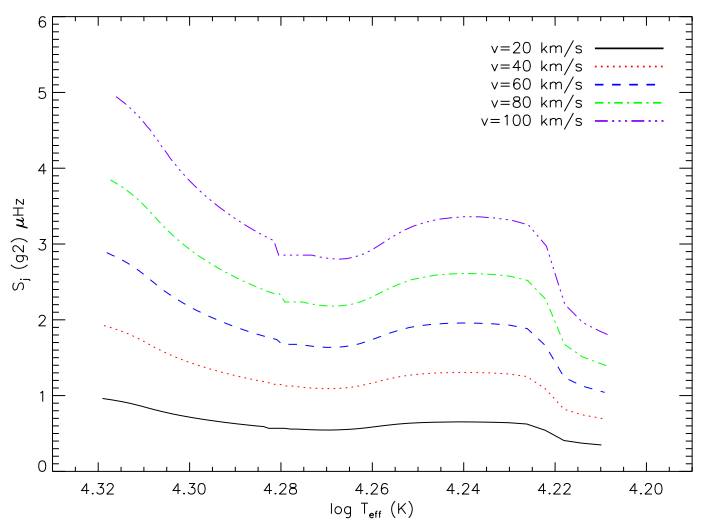

(b) $\mathrm{M}=7 M_{\odot}$

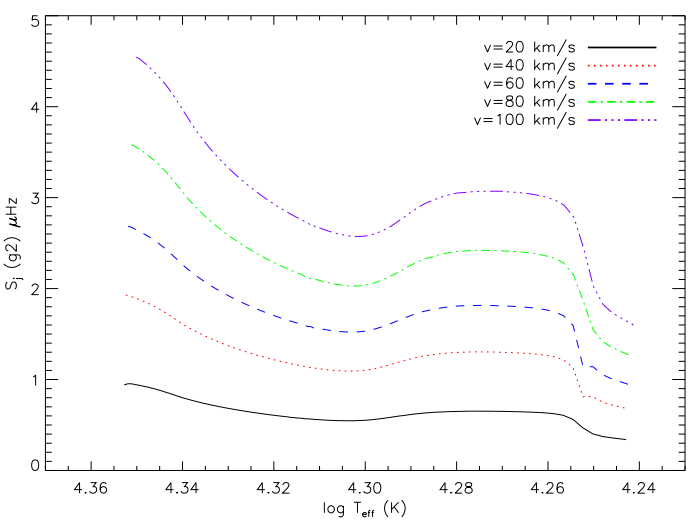

(c) $\mathrm{M}=8 M_{\odot}$

Figura 5.6: Idem a Figura 5.5, para as massas a) $6 M_{\odot}$, b) $7 M_{\odot}$ e c) $8 M_{\odot}$. 


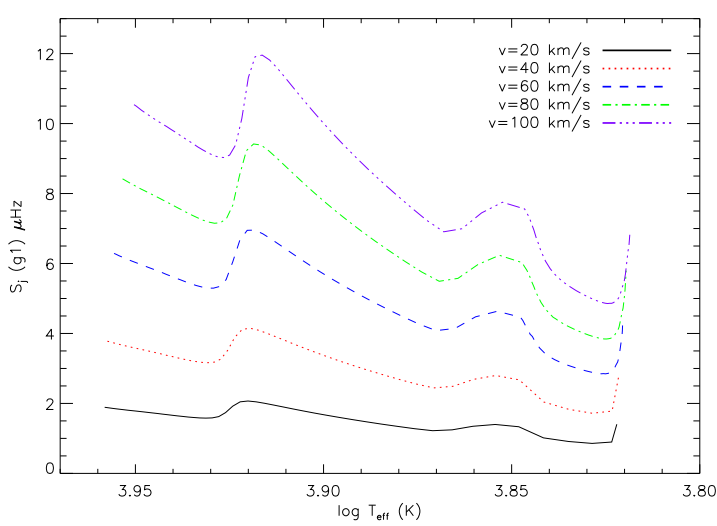

(a) $\mathrm{M}=2 M_{\odot}$

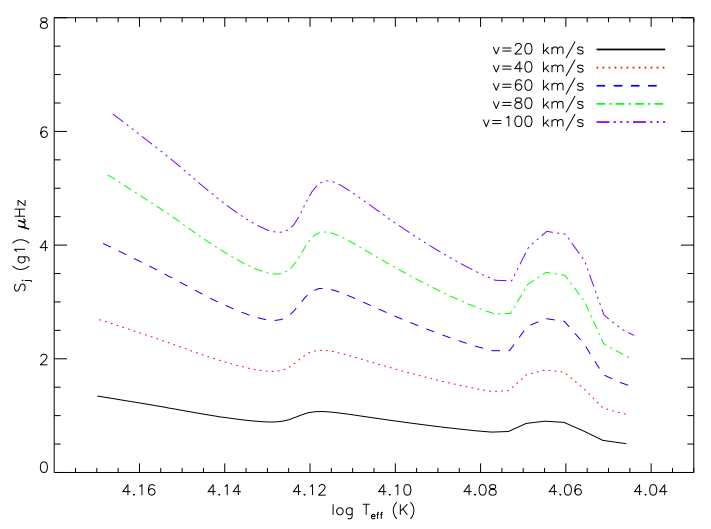

(c) $\mathrm{M}=4 M_{\odot}$

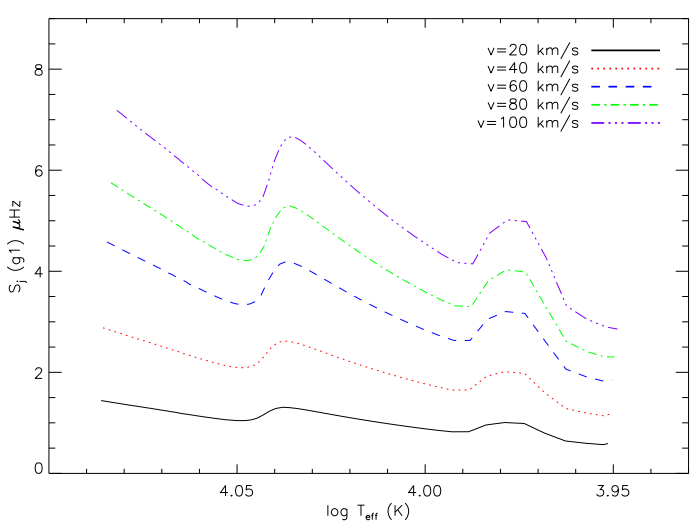

(b) $\mathrm{M}=3 M_{\odot}$

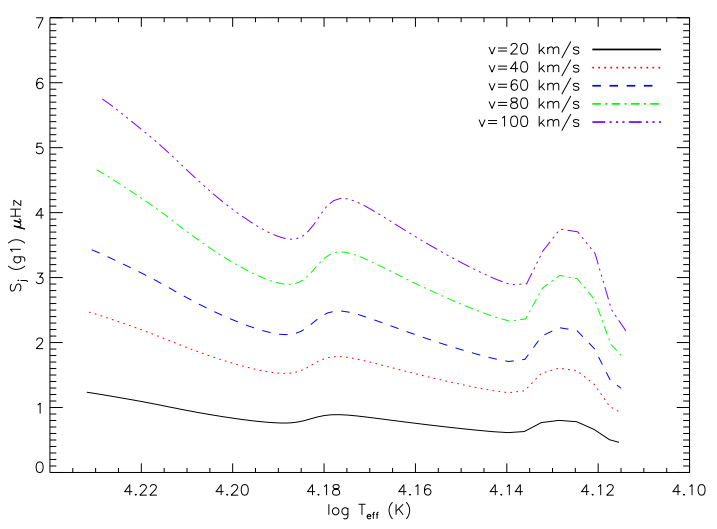

(d) $\mathrm{M}=5 M_{\odot}$

Figura 5.7: Idem a Figura 5.5, para o modo $g_{1}$ e, para as massas a) $2 M_{\odot}$, b) $3 M_{\odot}$, c) $4 M_{\odot}$ e d) $5 M_{\odot}$. 


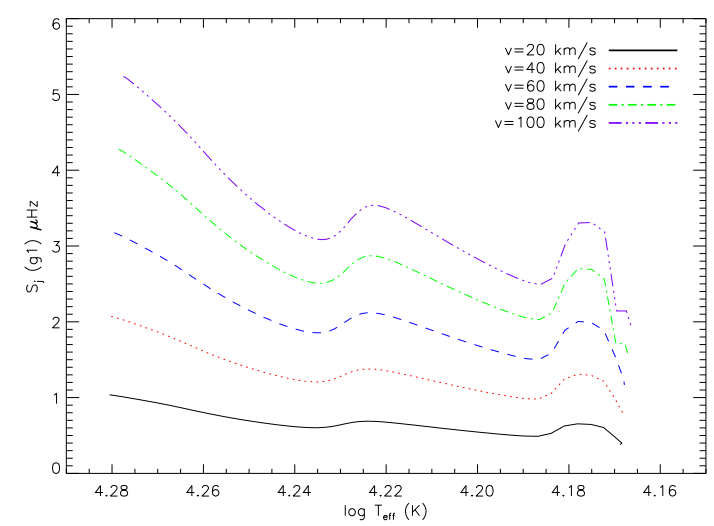

(a) $\mathrm{M}=6 M_{\odot}$

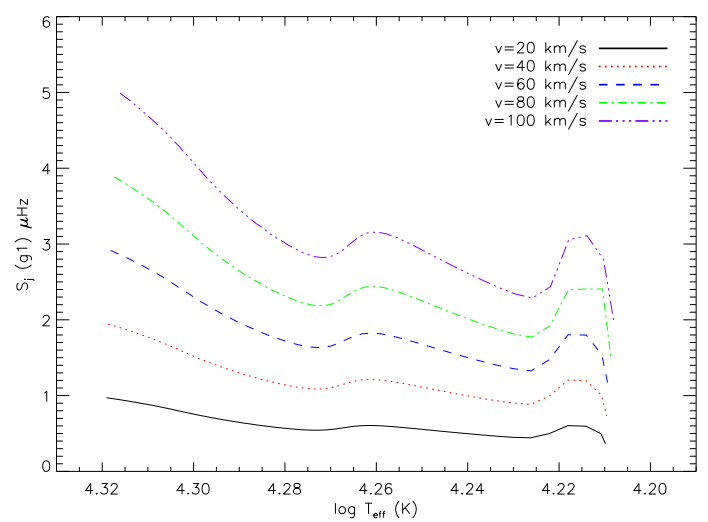

(b) $\mathrm{M}=7 M_{\odot}$

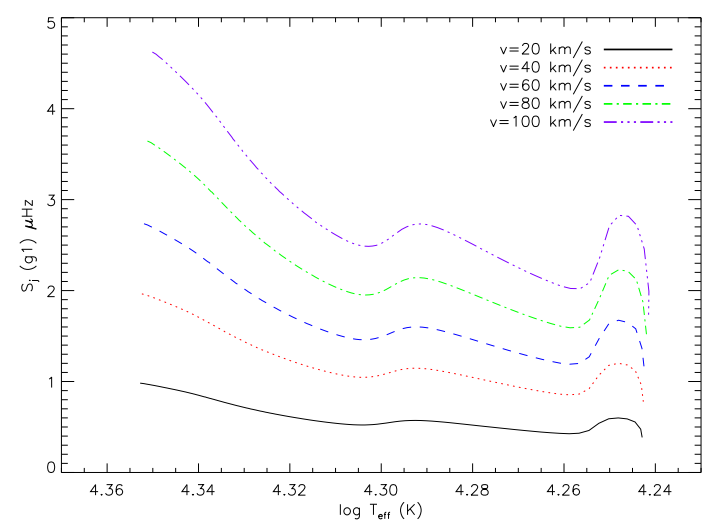

(c) $\mathrm{M}=8 M_{\odot}$

Figura 5.8: Idem a Figura 5.7, para as massas a) $6 M_{\odot}$, b) $7 M_{\odot}$ e c) $8 M_{\odot}$. 


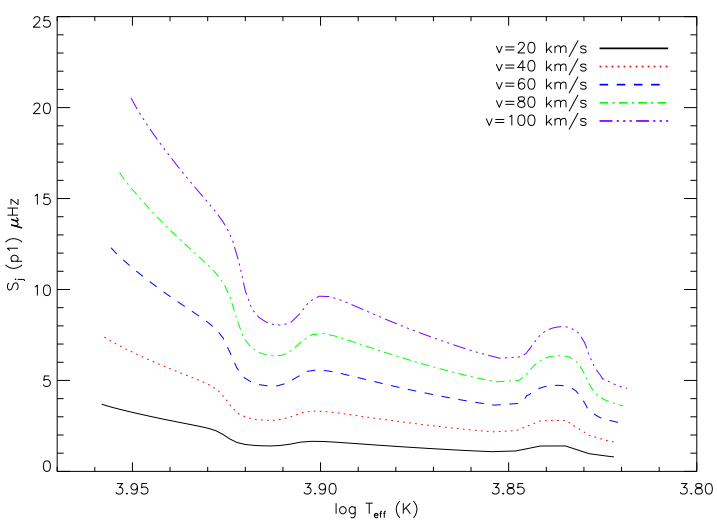

(a) $\mathrm{M}=2 M_{\odot}$

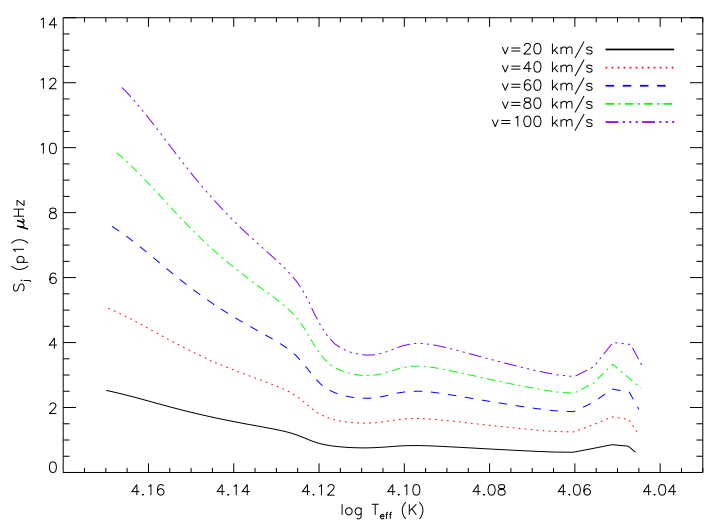

(c) $\mathrm{M}=4 M_{\odot}$

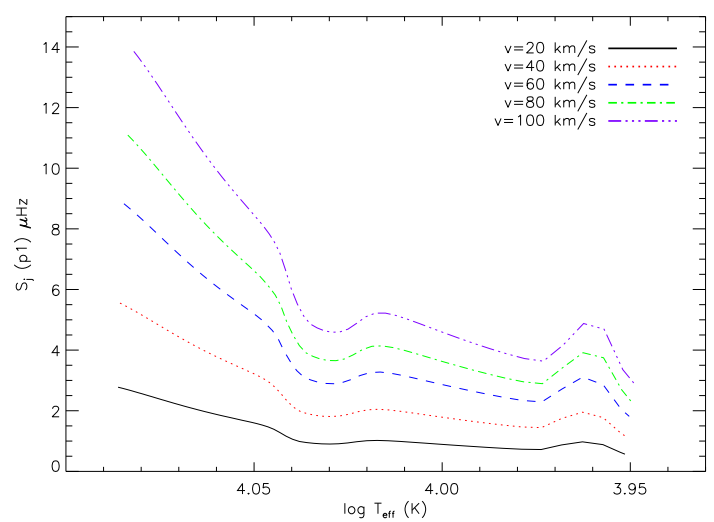

(b) $\mathrm{M}=3 M_{\odot}$

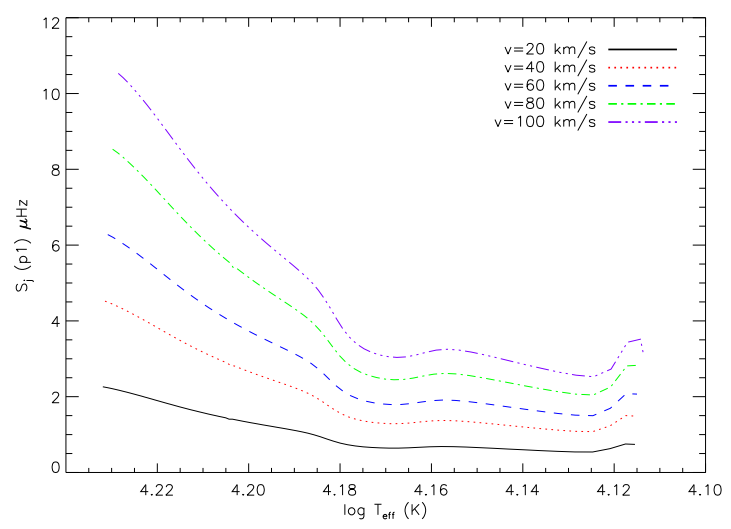

(d) $\mathrm{M}=5 M_{\odot}$

Figura 5.9: Idem a Figura 5.5, para o modo $p_{1}$ e, para as massas a) $2 M_{\odot}$, b) $3 M_{\odot}$, c) $4 M_{\odot}$ e d) $5 M_{\odot}$. 


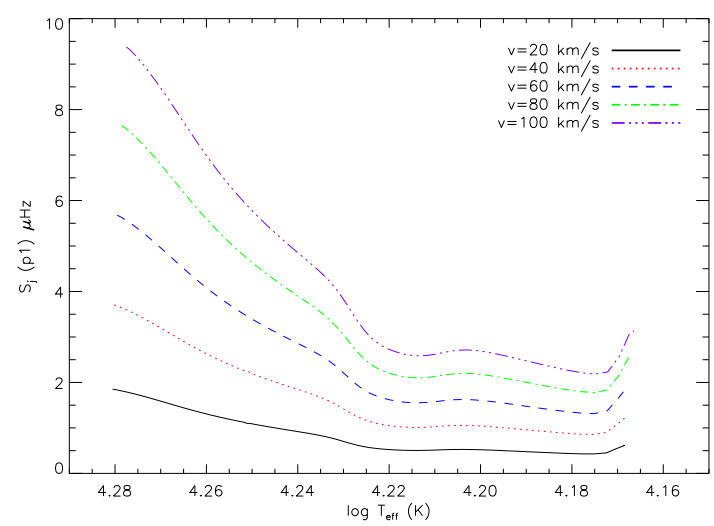

(a) $\mathrm{M}=6 M_{\odot}$

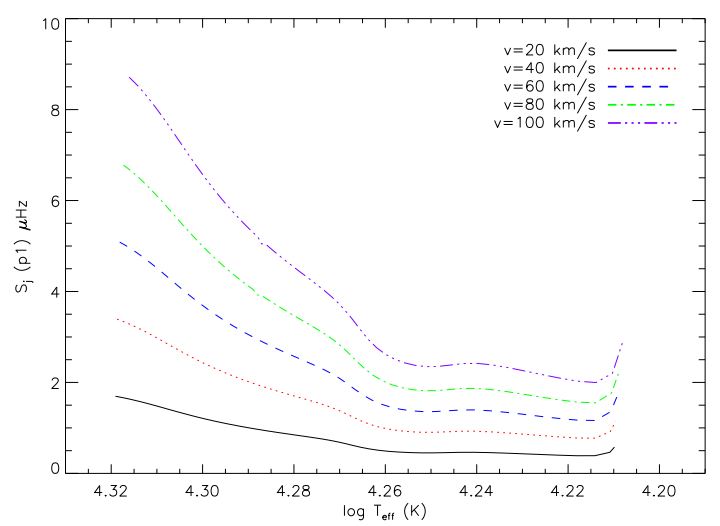

(b) $\mathrm{M}=7 M_{\odot}$

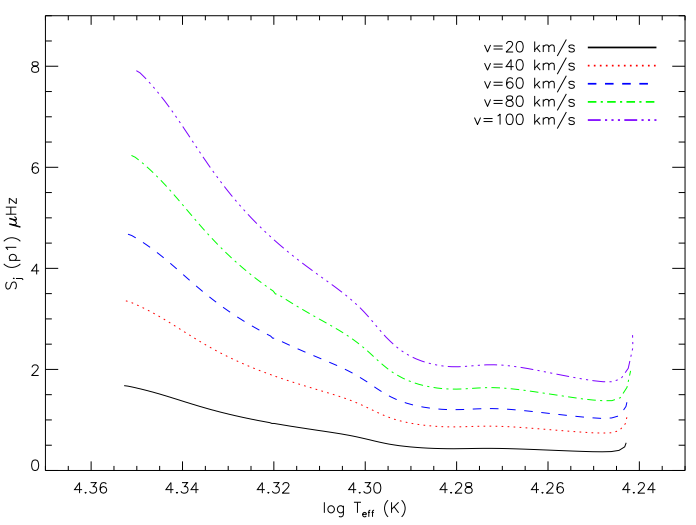

(c) $\mathrm{M}=8 M_{\odot}$

Figura 5.10: Idem a Figura 5.9, para as massas a) $6 M_{\odot}$, b) $7 M_{\odot}$ e c) $8 M_{\odot}$. 


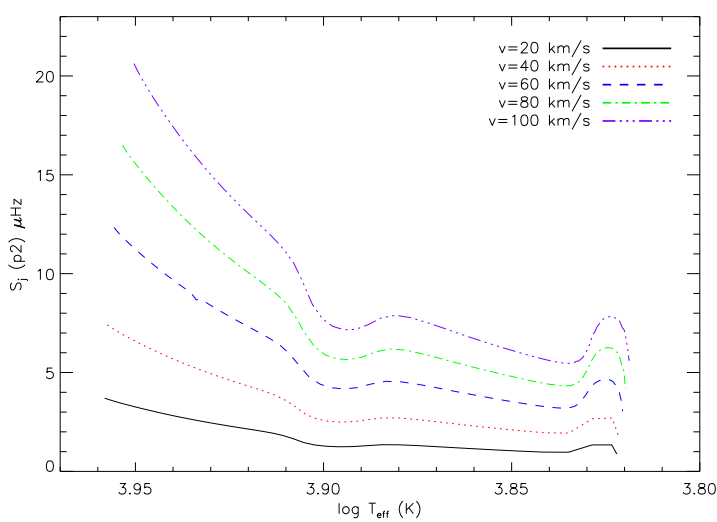

(a) $\mathrm{M}=2 M_{\odot}$

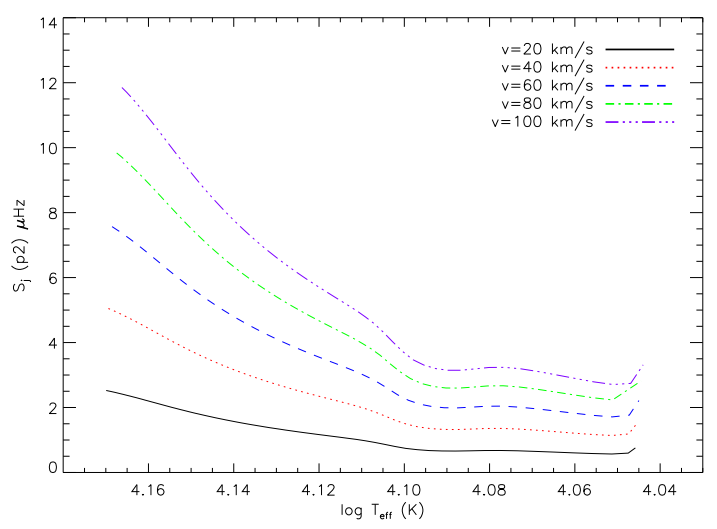

(c) $\mathrm{M}=4 M_{\odot}$

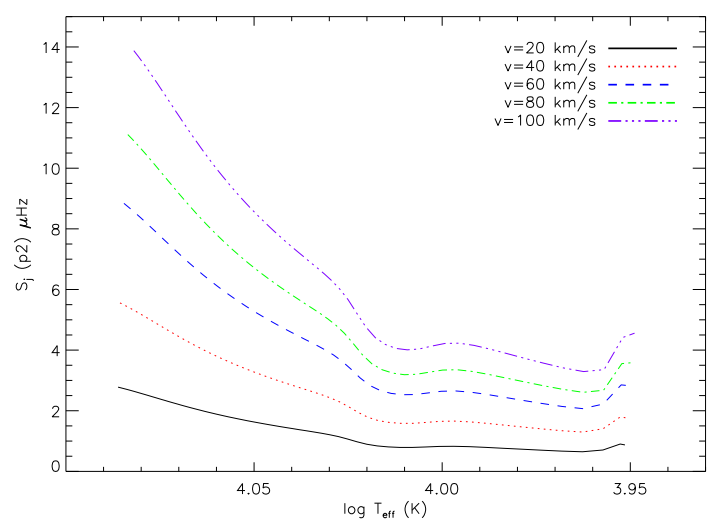

(b) $\mathrm{M}=3 M_{\odot}$

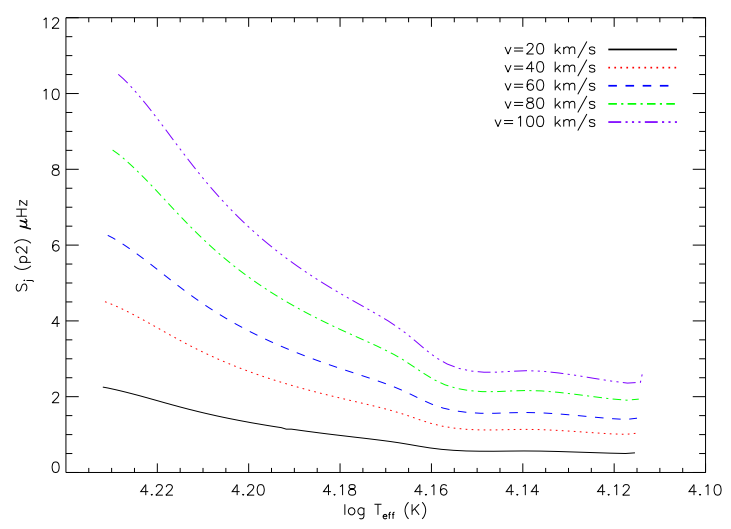

(d) $\mathrm{M}=5 M_{\odot}$

Figura 5.11: Idem a Figura 5.5, para o modo $p_{2}$ e, para as massas a) $2 M_{\odot}$, b) $3 M_{\odot}$, c) $4 M_{\odot}$ e d) $5 M_{\odot}$. 


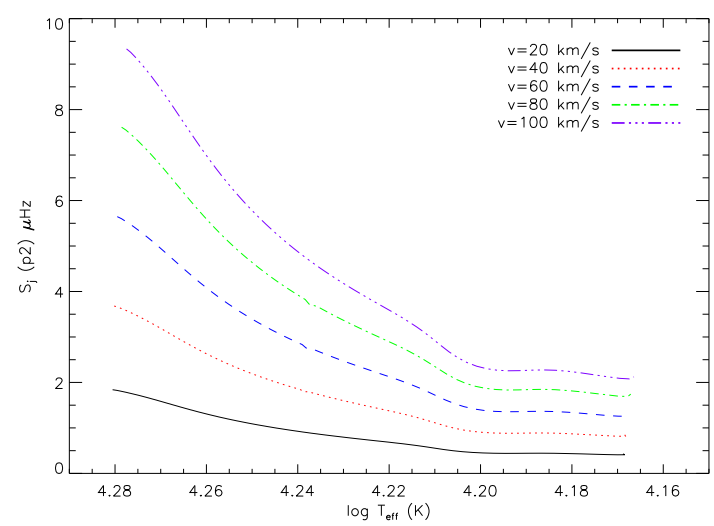

(a) $\mathrm{M}=6 M_{\odot}$

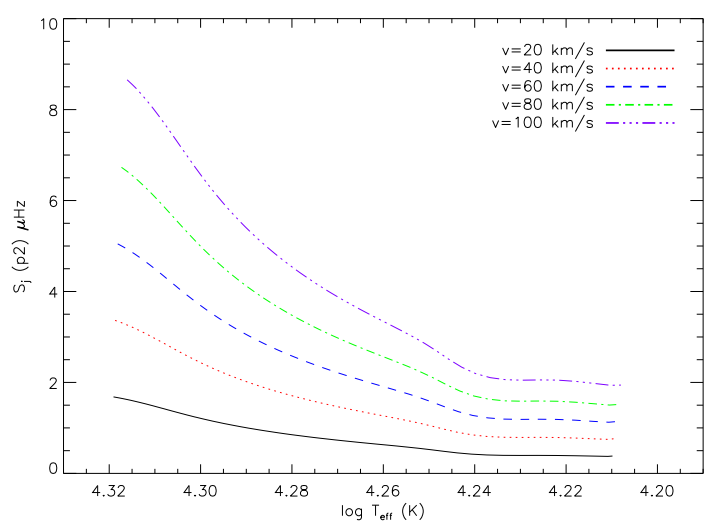

(b) $\mathrm{M}=7 M_{\odot}$

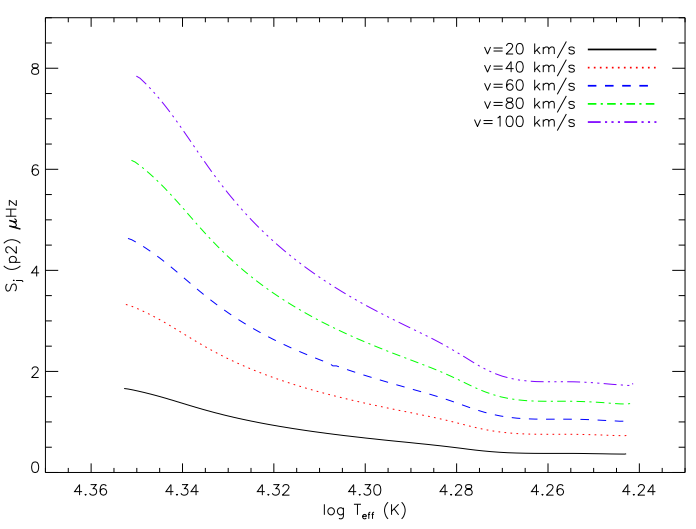

(c) $\mathrm{M}=8 M_{\odot}$

Figura 5.12: Idem a Figura 5.11, para as massas a) $6 M_{\odot}$, b) $7 M_{\odot}$ e c) $8 M_{\odot}$. 


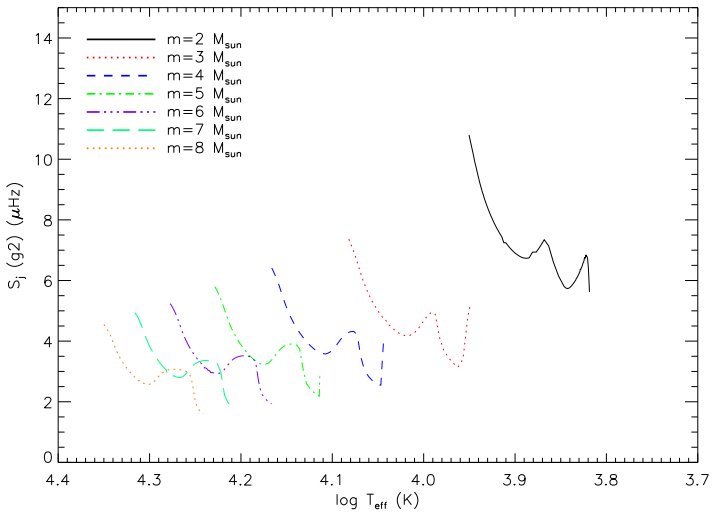

(a) modo g2

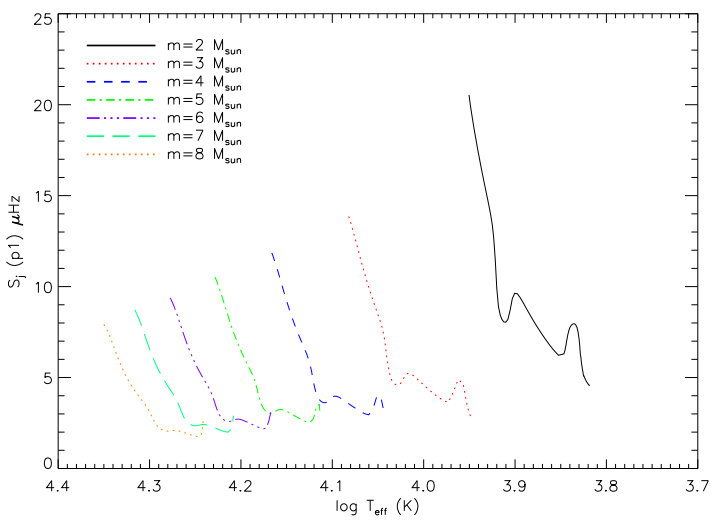

(c) modo $\mathrm{p} 1$

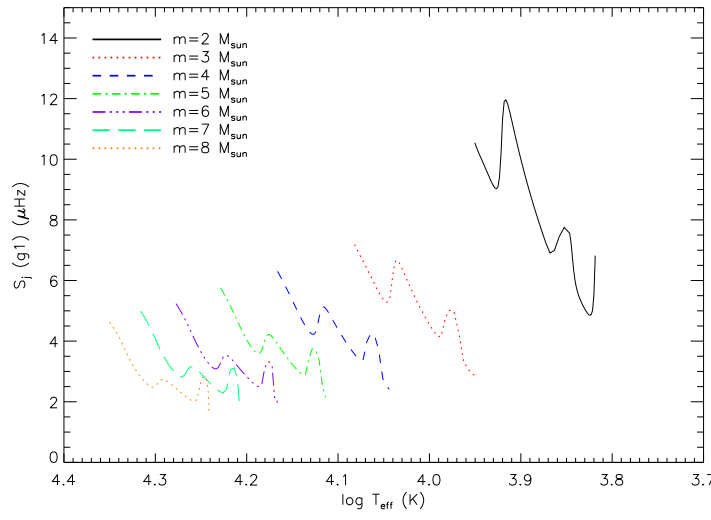

(b) modo g1

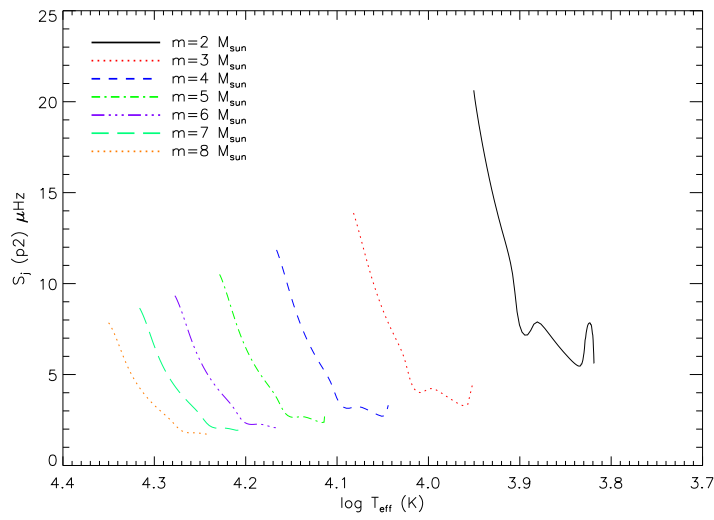

(d) modo p2

Figura 5.13: Comportamento do splitting rotacional dos modos a) $g_{2}$, b) $g_{1}$, c) $p_{1}$ e d) $p_{2}$ em função da temperatura efetiva para as trajetórias evolutivas das massas 2 à $8 M_{\odot}$ (direita para a esquerda) mostrando a influência da rotação com velocidade superficial de $100 \mathrm{~km} / \mathrm{s}$.

dade rotacional superficial de $100 \mathrm{~km} / \mathrm{s}$.

Para uma velocidade de rotação superficial maior, os modelos de menor massa apresentam valores de splitting maiores que para modelos de maior massa. Porém, ao comparar o caso para velocidades angulares de mesma ordem, esse efeito desaparece como mostra a Figura 5.14.

\subsection{Análise da Assimetria do Splitting Rotacional}

A deformação sofrida pela estrela com a introdução da rotação leva a assimetrias no splitting rotacional devido à não esfericidade da estrela como mencionado na seção 2.7.2. 


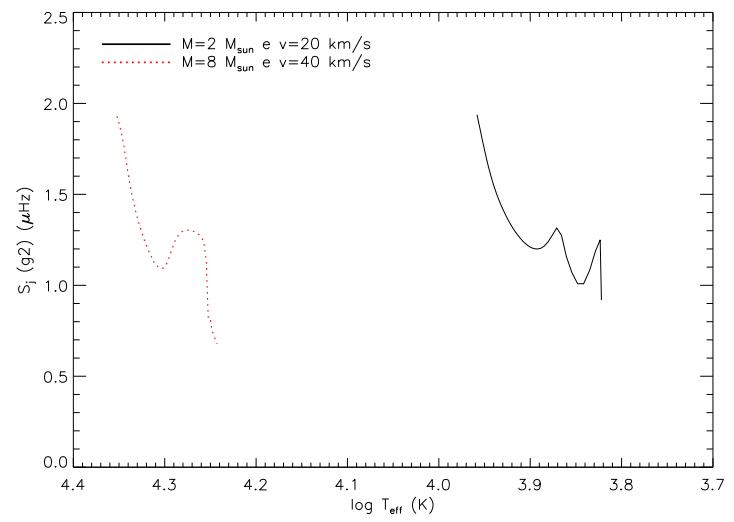

(a) modo g2

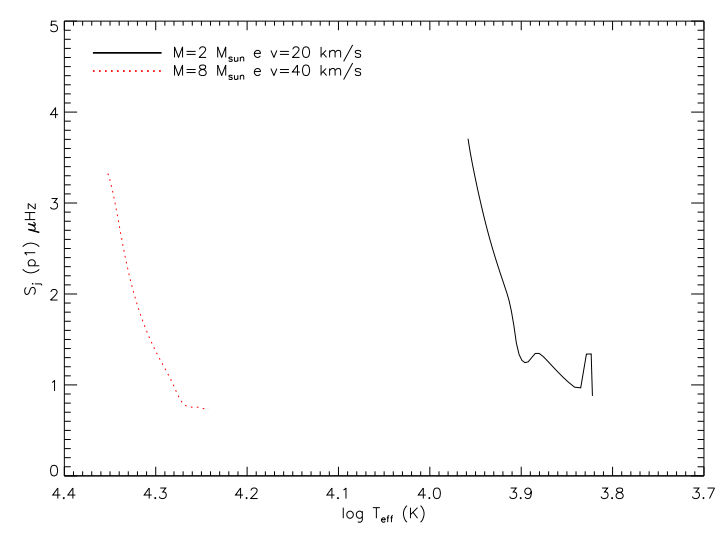

(c) modo p1

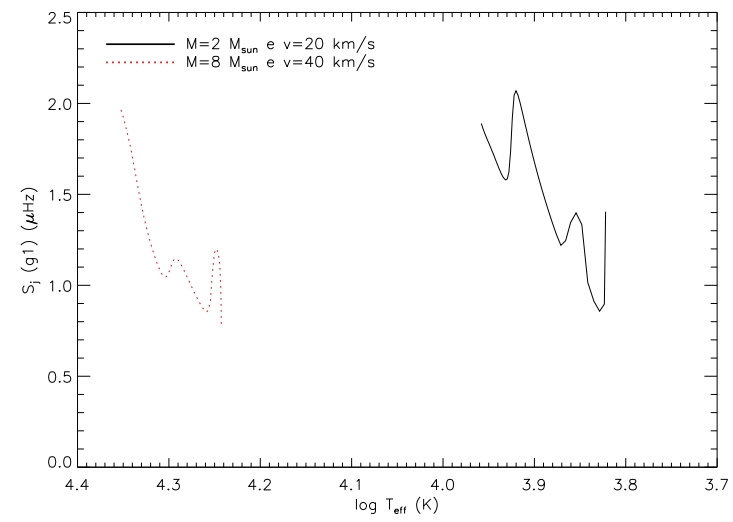

(b) modo g1

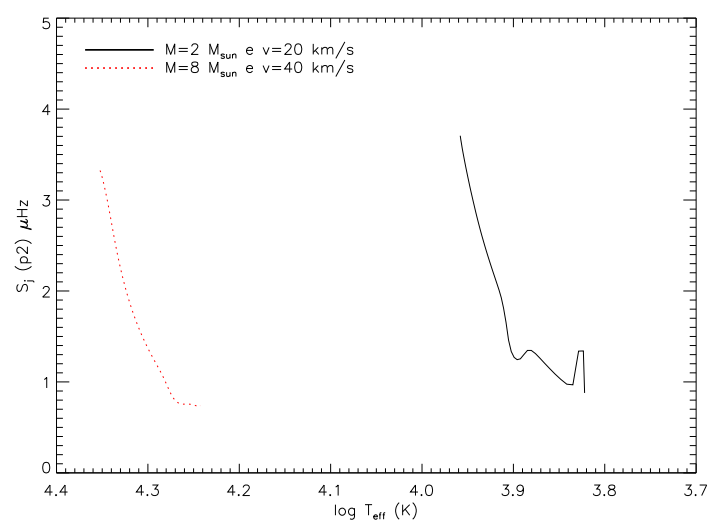

(d) modo p2

Figura 5.14: Comportamento do splitting rotacional dos modos a) $g_{2}$, b) $g_{1}$, c) $p_{1}$ e d) $p_{2}$ em função da temperatura efetiva para as trajetórias evolutivas mostrando a influência da rotação para velocidades angulares similares. A velocidade angular correspondente à $v_{r} o t=20 \mathrm{~km} / \mathrm{s}$ para a $2 M_{\odot}$ é igual a $2,3 \cdot 10^{-5}$ $\mathrm{rad} / \mathrm{s}$ e à $v_{r} o t=40 \mathrm{~km} / \mathrm{s}$ para $8 M_{\odot}$ é $2,1 \cdot 10^{-5} \mathrm{rad} / \mathrm{s}$. Note que a magnitude do splitting é da mesma ordem ao comparar modelos com velocidade angular próximas. 
A assimetria de um modo pode ser definida como

$$
A_{j}=\omega_{-1, j}+\omega_{+1, j}-2 \omega_{0, j}
$$

onde $\omega_{m, j}$ é a frequência do modo $\mathrm{j}$ com $\mathrm{m}=-1,0,+1$.

As Figuras 5.15 a 5.22 mostram o comportamento da assimetria em função da temperatura efetiva para os modos $g_{2}, g_{1}, p_{1}$ e $p_{2}$ e para a faixa de massas analisada.

O comportamento da assimetria para o modo $g_{2}$ para o caso de baixa massa, apresenta pontos em que a assimetria vai a zero independentemente da velocidade de rotação. Isso ocorre também no caso dos modos $g_{1}$ para todas as massas e no caso do modo $p_{1}$ para baixas massas. Desse modo, verifica-se que o fato de um modo não apresentar assimetria não diz a rigor nada com respeito à sua velocidade de rotação. Mais que isso, não é possível identificar em que fase evolutiva a estrela se encontra, se existe mais de um ponto onde isso ocorre. Além disso, mostra que a assimetria não é apenas medida da não-esfericidade da estrela. De fato, a assimetria aparece com as correções de segunda ordem e dependem de diversos parâmetros físicos da estrela além da velocidade de rotação como mostra a equação 2.146 .

Durante a fase na sequência principal, o principal parâmetro físico que sofre mudança é a composição química do núcleo convectivo. Como a assimetria desaparece para os modos, em alguns casos, em dois momentos na trajetória evolutiva, só a mudança desse parâmetro físico não é suficiente para explicar. Provavelmente existe uma combinação de fatores que levam a esse comportamento.

Em geral, a assimetria dos modos tem comportamento similar para todas as massas, porém torna-se mais suave com o aumento da massa. Esse comportamento pode ser visualizado melhor na Figura 5.23 para o caso com velocidade de rotação superficial de $100 \mathrm{~km} / \mathrm{s}$. Entre os modos analisados, o modo $g_{1}$ é o que apresenta o comportamento mais peculiar. A assimetria desse modo ao longo da evolução apresenta vários pontos de retorno e momentos em que a assimetria muda de sinal. Isso ocorre para todas a faixa de massas analisadas.

Esse comportamento peculiar da assimetria é interessante e pode ser utilizado como diagnóstico para a evolução. O método que descobrimos consiste no cálculo de modelos representativos das estrelas considerando as caixas de erro dos parâmetros físicos determi- 


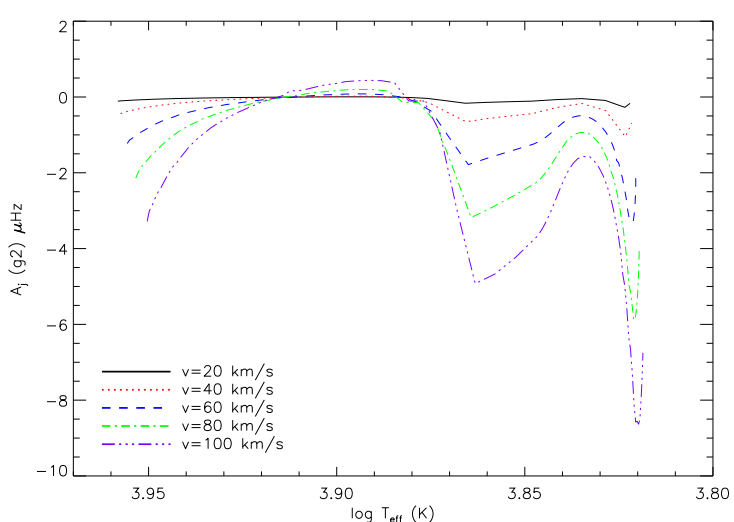

(a) $\mathrm{M}=2 M_{\odot}$

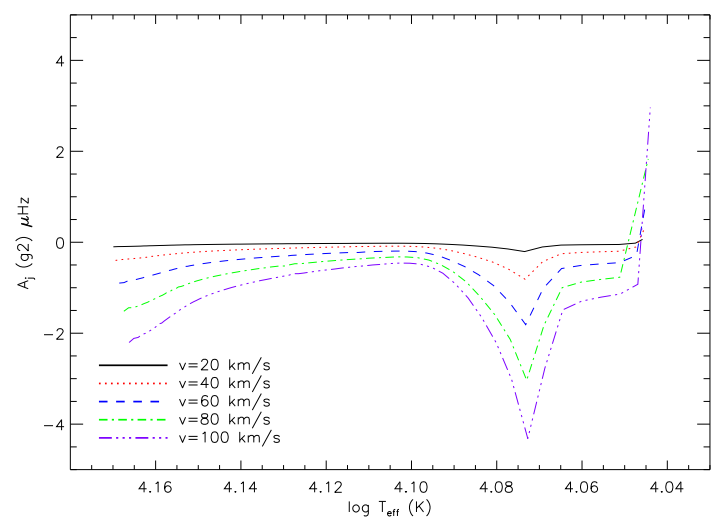

(c) $\mathrm{M}=4 M_{\odot}$

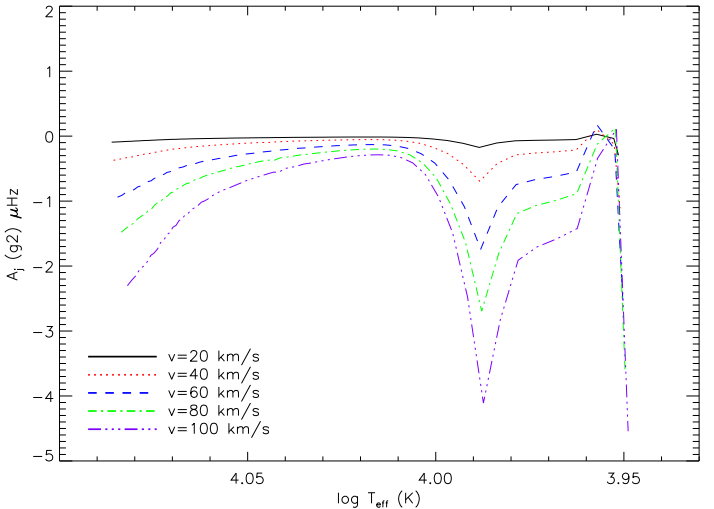

(b) $\mathrm{M}=3 M_{\odot}$

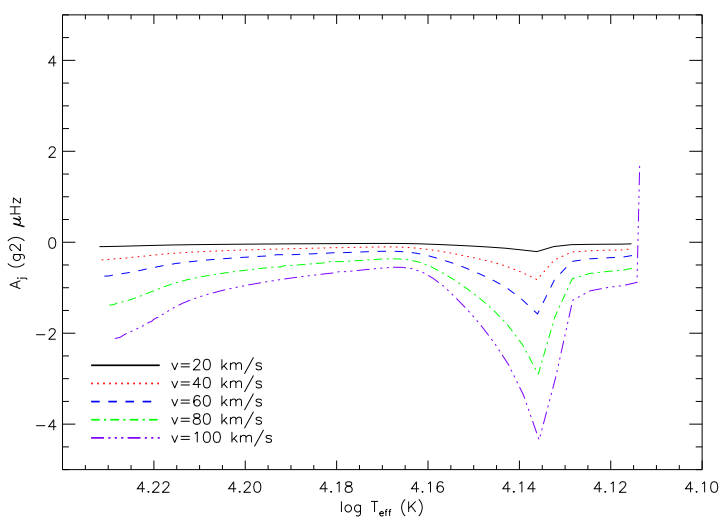

(d) $\mathrm{M}=5 M_{\odot}$

Figura 5.15: Comportamento do assimetria do splitting rotacional do modo $g_{2}$ em função da temperatura efetiva para as trajetórias evolutivas das massas a) $2 M_{\odot}$, b) $3 M_{\odot}$, c) $4 M_{\odot}$ e d) $5 M_{\odot}$ mostrando a influência da rotação com velocidade superficial de 20, 40, 60, 80 e $100 \mathrm{~km} / \mathrm{s}$. Existe um problema de convergência dos códigos utilizados nos pontos à direita (picos) das Figuras c) e d). Note que uma ausência de assimetria pode indicar que a estrela esta numa determinada fase de evolução, onde a assimetria tende a se anular para esse modo, como p. ex., no quadro a) em $\log T_{\text {eff }} \sim 3,91 ; 3,87$ e, eventualmente 3.83 . 


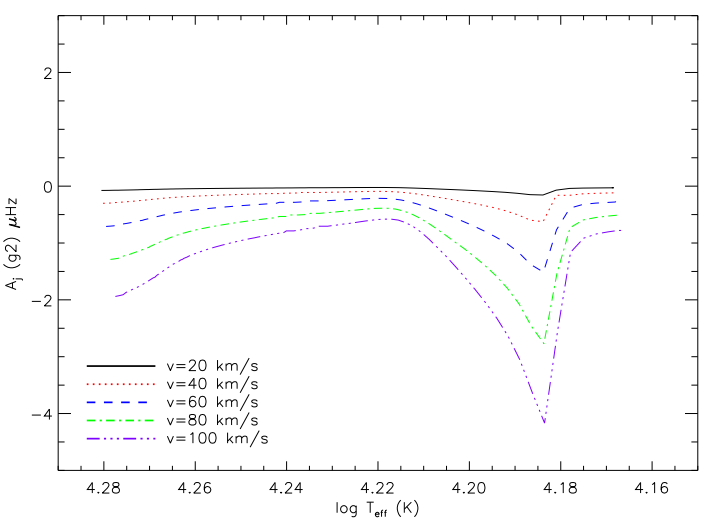

(a) $\mathrm{M}=6 M_{\odot}$

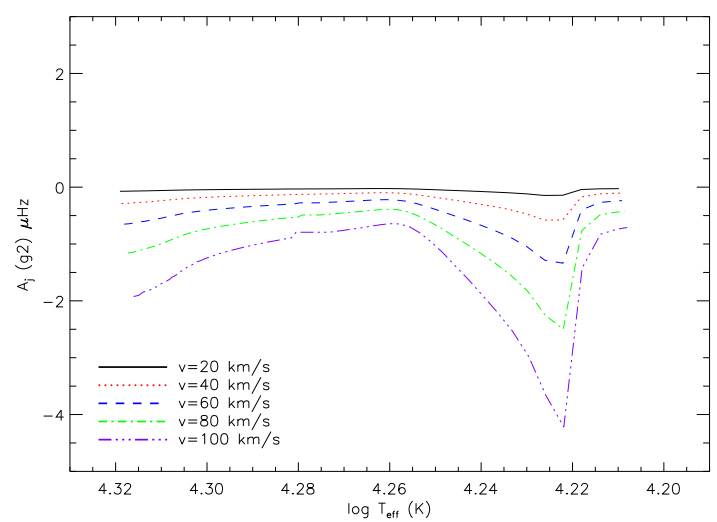

(b) $\mathrm{M}=7 M_{\odot}$

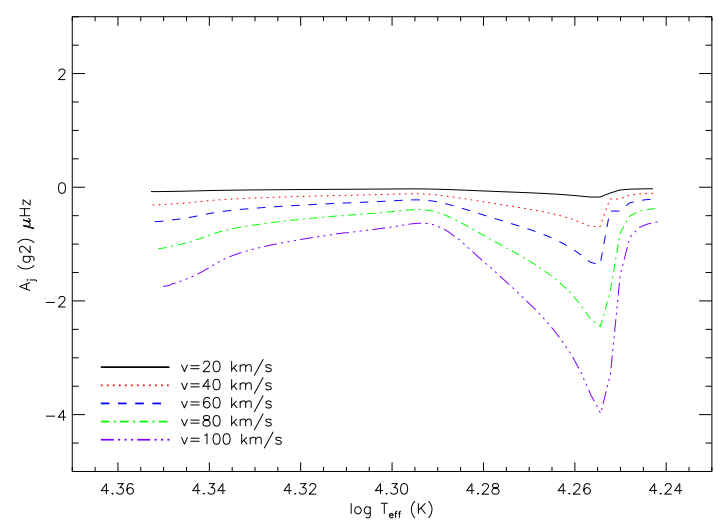

(c) $\mathrm{M}=8 M_{\odot}$

Figura 5.16: Idem a Figura 5.15, para as massas a) $6 M_{\odot}$, b) $7 M_{\odot}$ e c) $8 M_{\odot}$. 


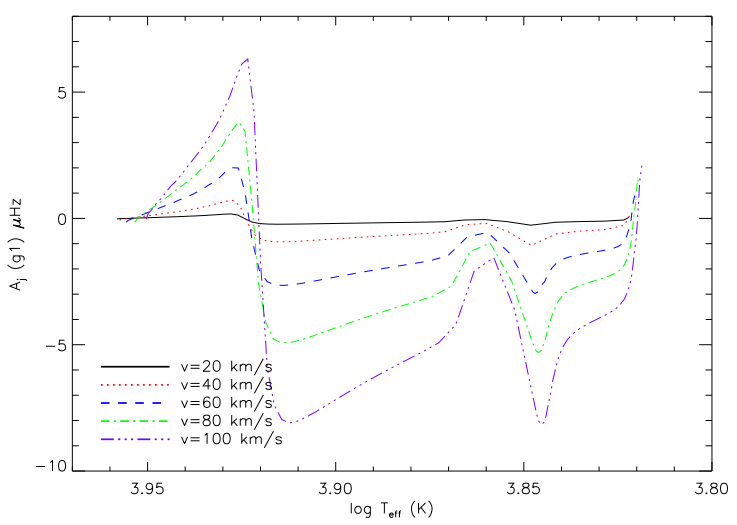

(a) $\mathrm{M}=2 M_{\odot}$

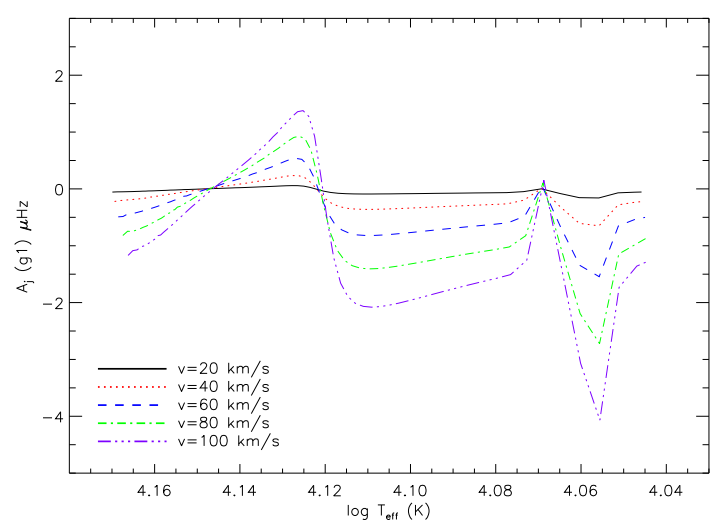

(c) $\mathrm{M}=4 M_{\odot}$

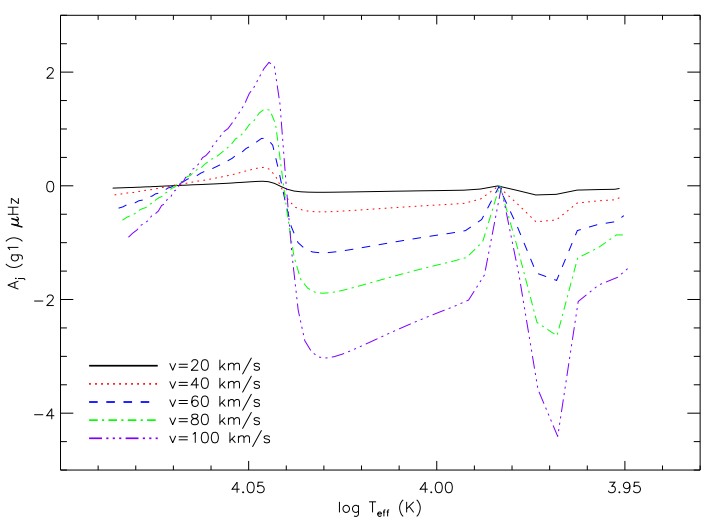

(b) $\mathrm{M}=3 M_{\odot}$

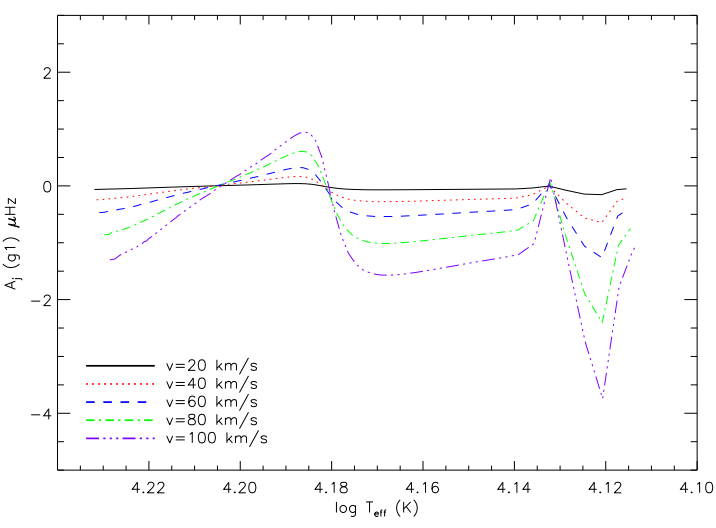

(d) $\mathrm{M}=5 M_{\odot}$

Figura 5.17: Idem a Figura 5.15, para o modo $g_{1}$ e, para as massas a) $2 M_{\odot}$, b) $3 M_{\odot}$, c) $4 M_{\odot}$ e d) $5 M_{\odot}$. Note que uma ausência de assimetria pode indicar que a estrela esta numa determinada fase de evolução, onde a assimetria tende a se anular para esse modo, como p. ex., no quadro a) em $\log T_{\text {eff }} \sim 3,95 ; 3,92$ e, eventualmente 3,86 . 


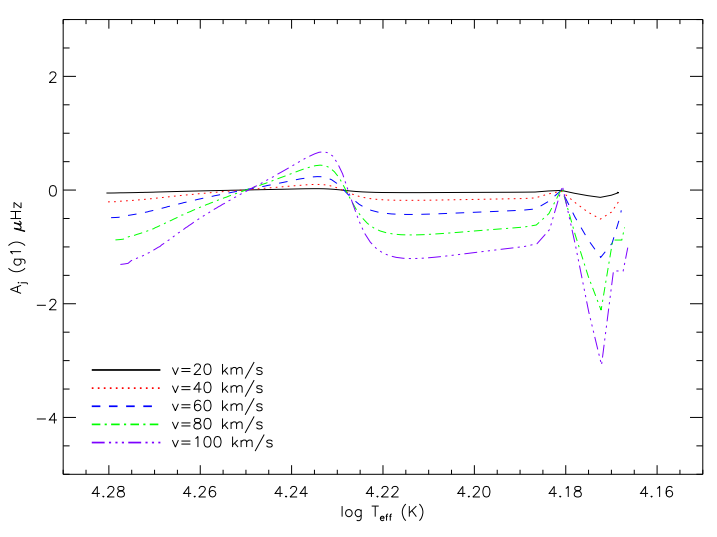

(a) $\mathrm{M}=6 M_{\odot}$

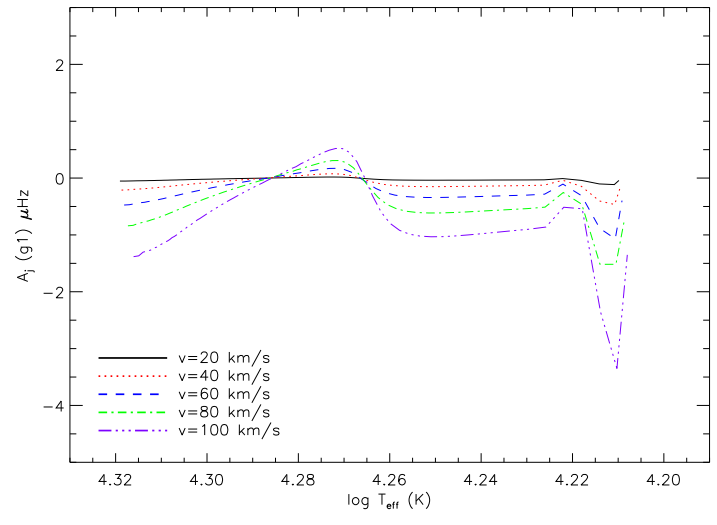

(b) $\mathrm{M}=7 M_{\odot}$

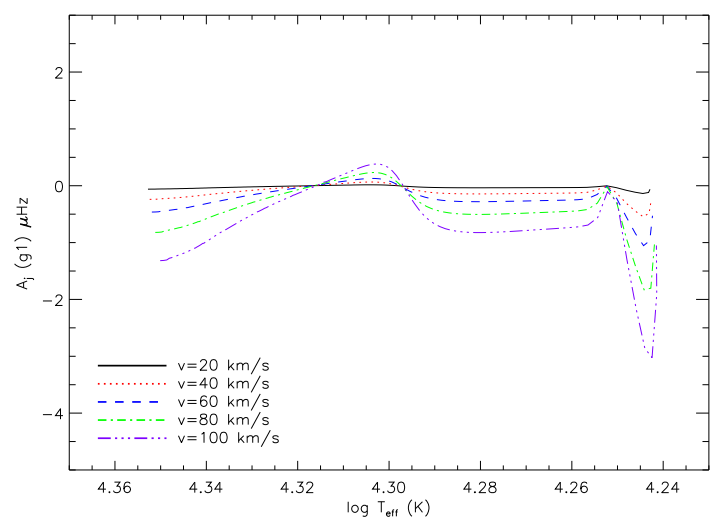

(c) $\mathrm{M}=8 M_{\odot}$

Figura 5.18: Idem a Figura 5.17, para as massas a) $6 M_{\odot}$, b) $7 M_{\odot}$ e c) $8 M_{\odot}$. Note que uma ausência de assimetria pode indicar que a estrela esta numa determinada fase de evolução, onde a assimetria tende a se anular para esse modo, como p. ex., no quadro a) em $\log T_{\text {eff }} \sim 4,25 ; 4,23$ e 3.18 . 


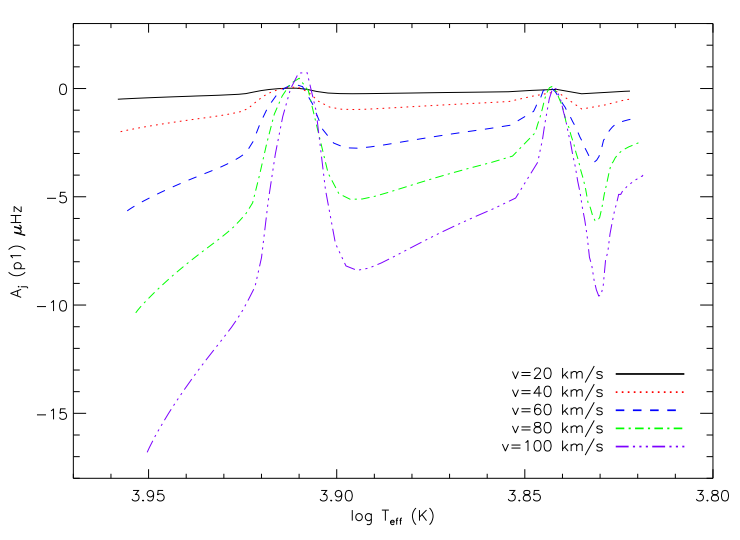

(a) $\mathrm{M}=2 M_{\odot}$

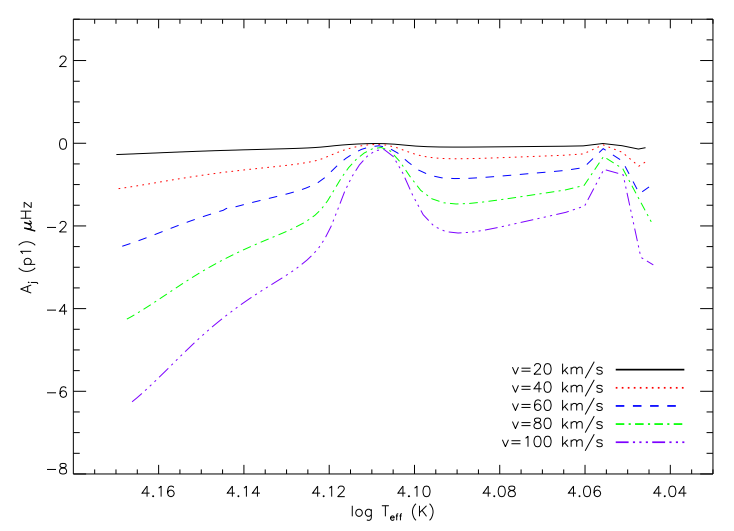

(c) $\mathrm{M}=4 M_{\odot}$

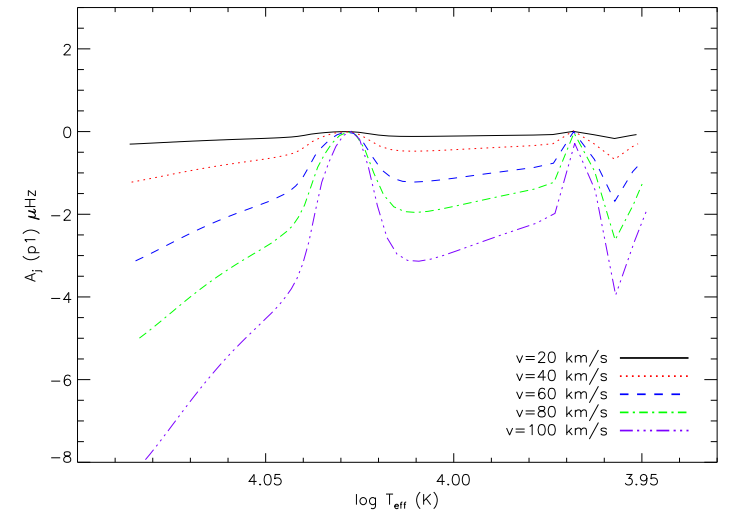

(b) $\mathrm{M}=3 M_{\odot}$

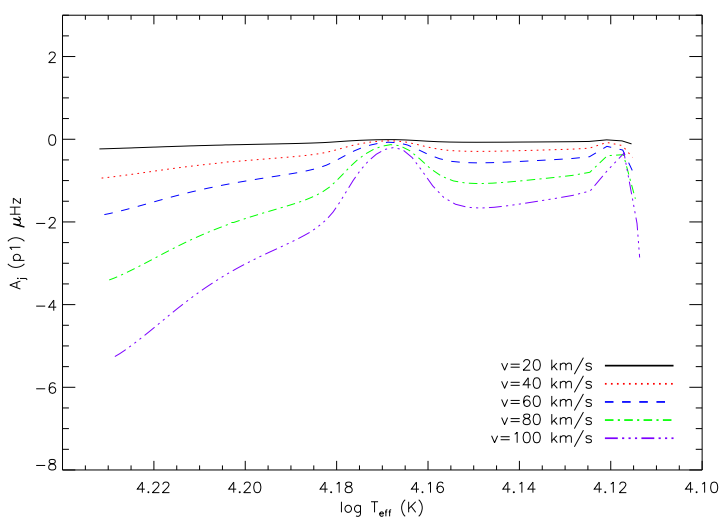

(d) $\mathrm{M}=5 M_{\odot}$

Figura 5.19: Idem a Figura 5.15, para o modo $p_{1}$ e, para as massas a) $2 M_{\odot}$, b) $3 M_{\odot}$, c) $4 M_{\odot}$ e d) $5 M_{\odot}$. Note que uma ausência de assimetria pode indicar que a estrela esta numa determinada fase de evolução, onde a assimetria tende a se anular para esse modo, como p. ex., no quadro a) em $\log T_{\text {eff }} \sim 3,91$ e 3,84 . 


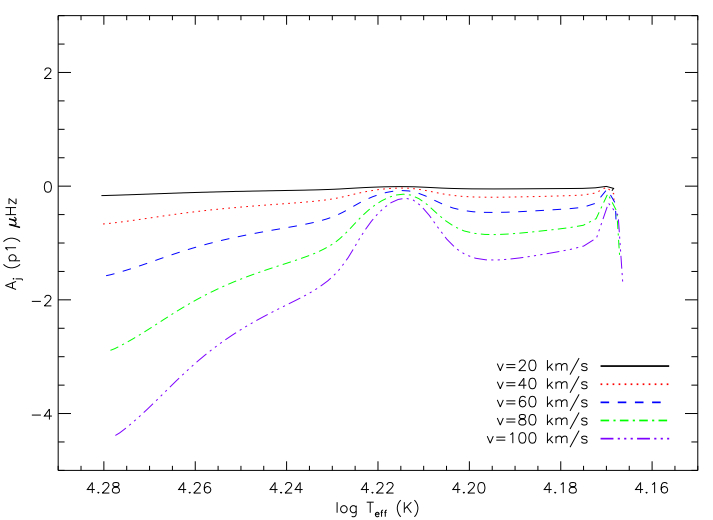

(a) $\mathrm{M}=6 M_{\odot}$

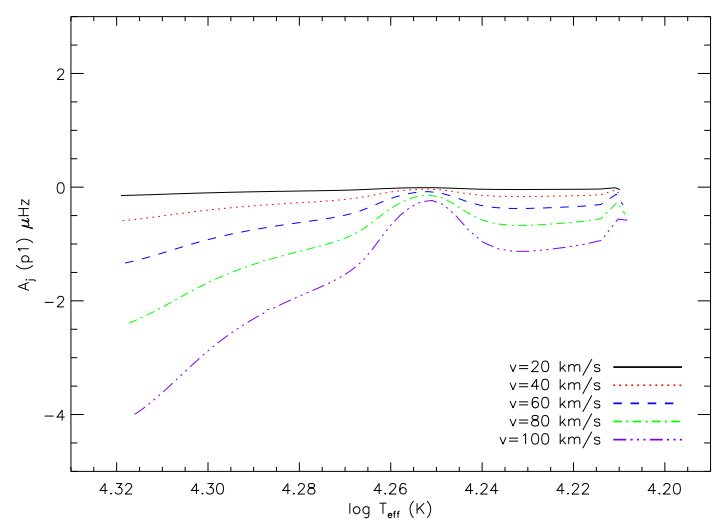

(b) $\mathrm{M}=7 M_{\odot}$

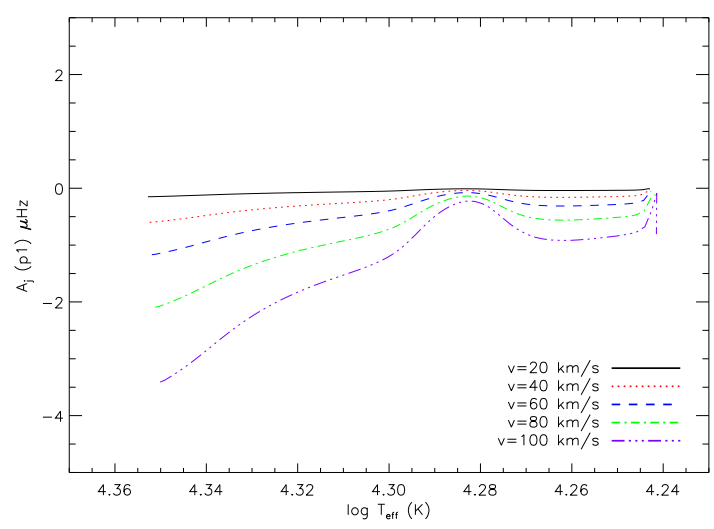

(c) $\mathrm{M}=8 M_{\odot}$

Figura 5.20: Idem a Figura 5.19, para as massas a) $6 M_{\odot}$, b) $7 M_{\odot}$ e c) $8 M_{\odot}$. 


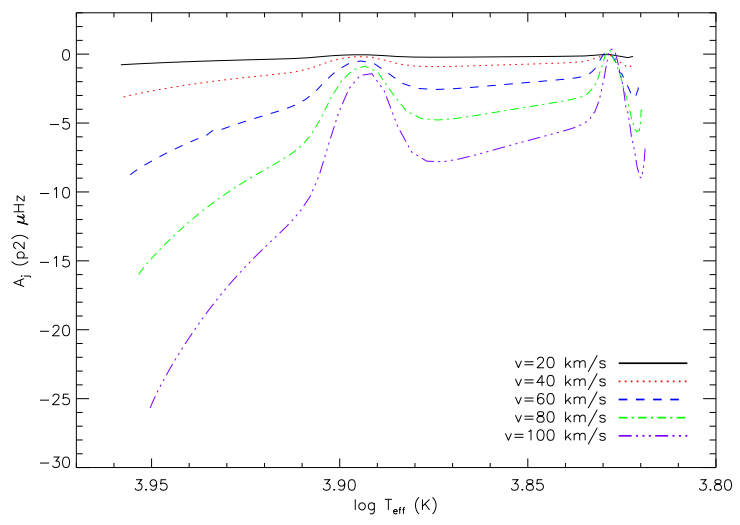

(a) $\mathrm{M}=2 M_{\odot}$

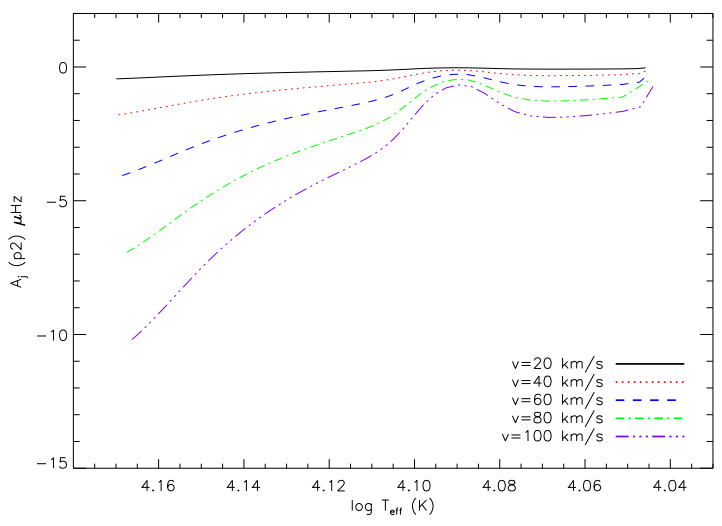

(c) $\mathrm{M}=4 M_{\odot}$

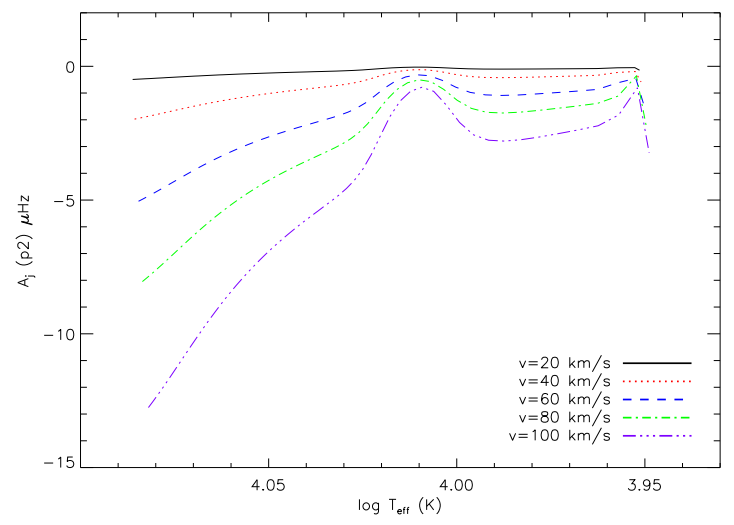

(b) $\mathrm{M}=3 M_{\odot}$

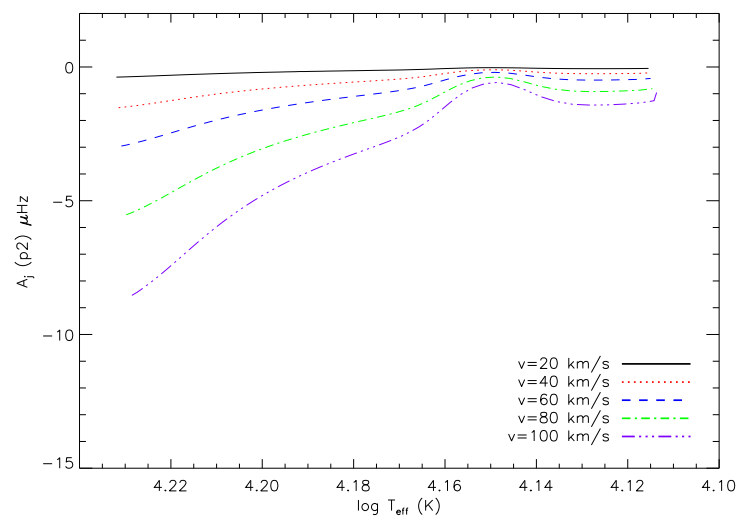

(d) $\mathrm{M}=5 M_{\odot}$

Figura 5.21: Idem a Figura 5.15, para o modo $p_{2}$ e, para as massas a) $2 M_{\odot}$, b) $3 M_{\odot}$, c) $4 M_{\odot}$ e d) 5 $M_{\odot}$. 


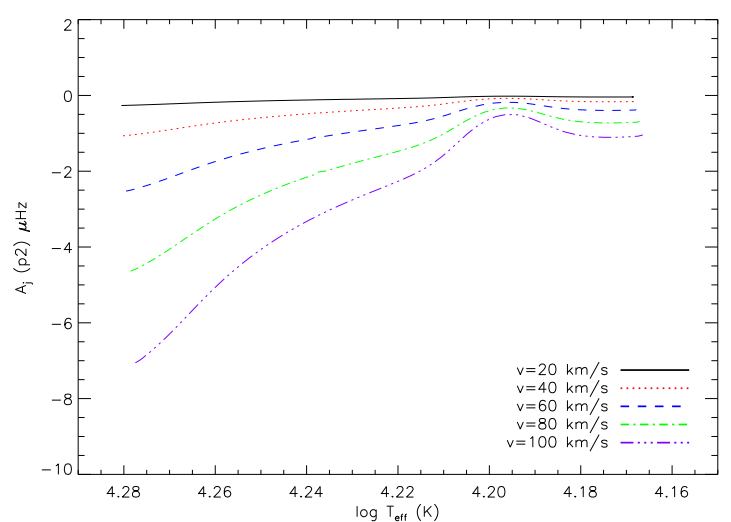

(a) $\mathrm{M}=6 M_{\odot}$

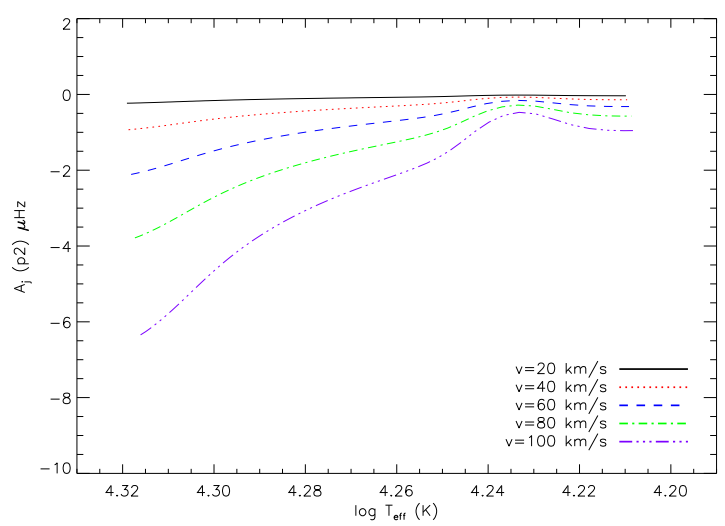

(b) $\mathrm{M}=7 M_{\odot}$

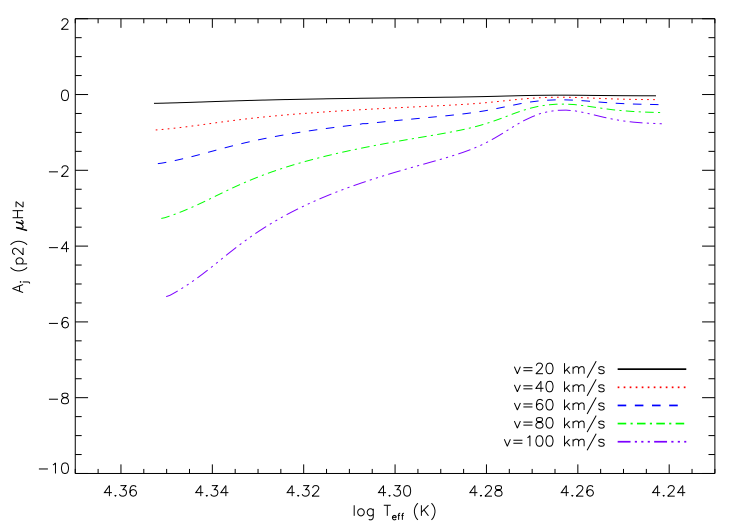

(c) $\mathrm{M}=8 M_{\odot}$

Figura 5.22: Idem a Figura 5.21, para as massas a) $6 M_{\odot}$, b) $7 M_{\odot}$ e c) $8 M_{\odot}$. 


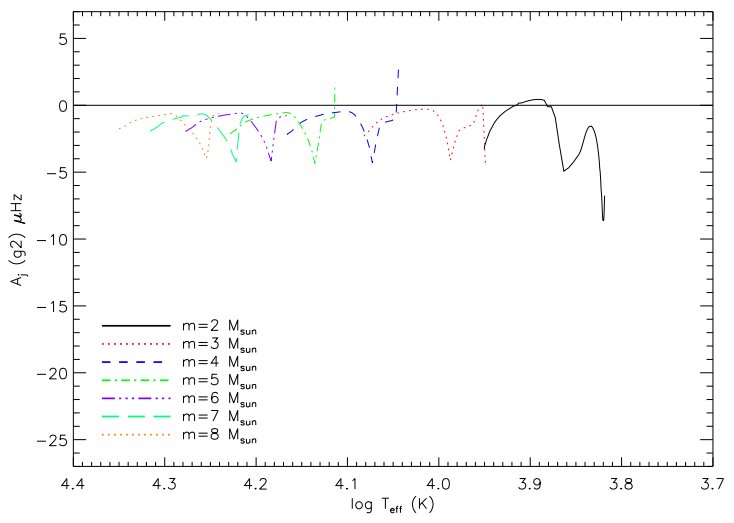

(a) modo g2

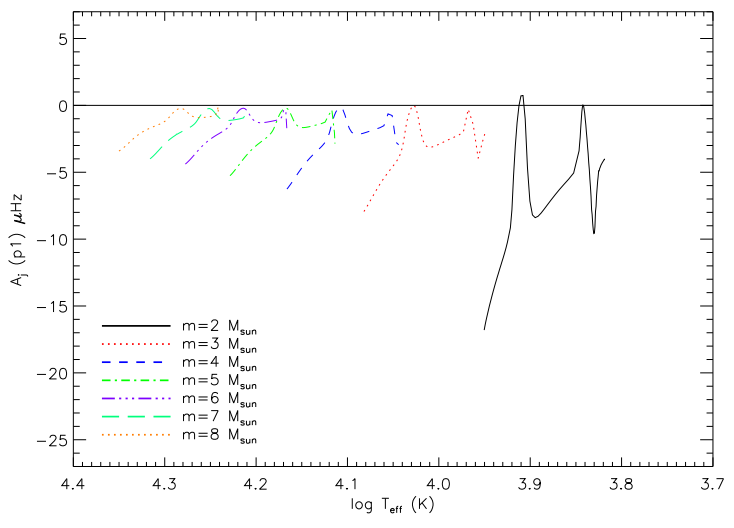

(c) modo p1

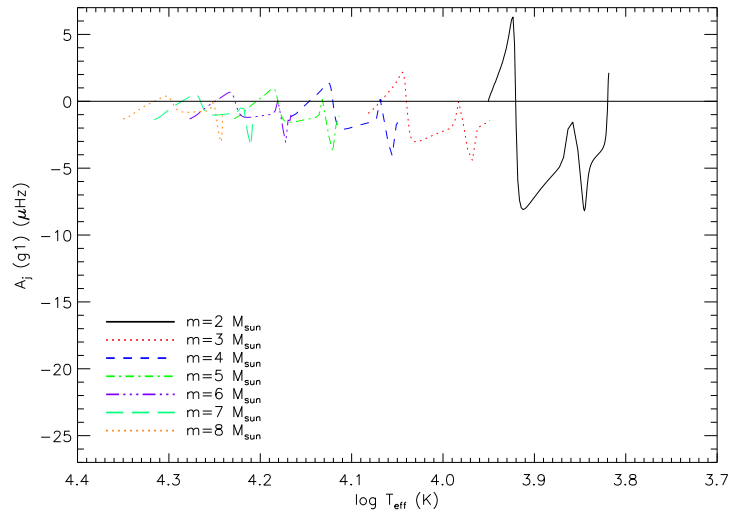

(b) modo g1

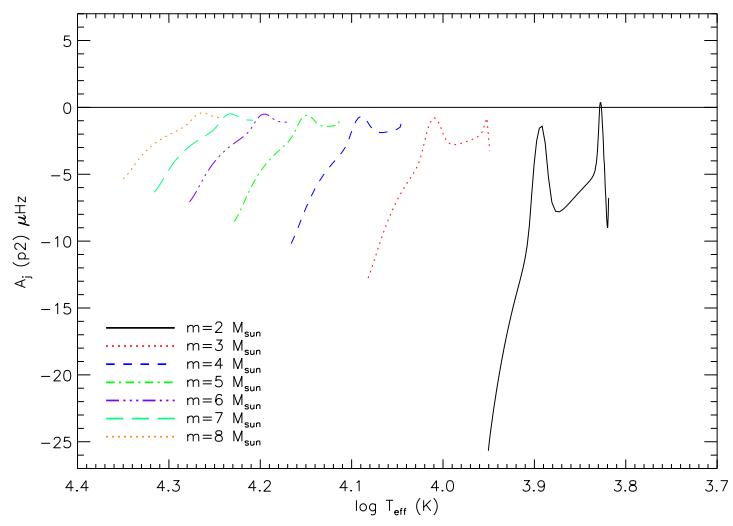

(d) modo p2

Figura 5.23: Comportamento da assimetria do splitting rotacional dos modos a) $g_{2}$, b) $g_{1}$, c) $p_{1}$ e d) $p_{2}$ em função da temperatura efetiva para as trajetórias evolutivas das massas 2 a $8 M_{\odot}$ (direita para a esquerda) mostrando a influência da rotação com velocidade superficial de 100 km/s.

nados. Com a identificação da assimetria para mais de um modo, é possível determinar com grande precisão a idade da estrela. Por exemplo, para estrelas de $2 M_{\odot}$, a identificação da ausência de assimetria no modo $g_{2}$ (Figura 5.15a) e no modo $p_{1}$ (Figura 5.19a) leva a determinação da $T_{\text {eff }} \sim 3,91$. Isso mostra também a importância do método sismológico. No diagrama HR, as mudanças estruturais são suaves e apontam na mesma direção para os modelos de diferentes massas. O que permite diferenciá-los é justamente a observação e identificação das frequências.

Assim como para o splitting, a assimetria parece ter maior intensidade para modelos de menor massa do que para modelos de maior massa, considerando a mesma velocidade. No entanto, ao comparar estrelas para velocidades angulares de mesma ordem, esse efeito 
desaparece como mostram as Figuras 5.24.

\subsection{Comparação com a estrela HD50844}

A estrela $\delta$ Scuti HD50844 foi observada com o satélite CoRoT durante a observação inicial (IR01) entre 2 de fevereiro de 2007 e 31 de março de 2007. O tempo de exposição no canal de sismologia estelar é de 1 segundo. Seu espectro de frequência foi analisado e publicado por Poretti et al. (2009), que obteve as frequências de oscilação. Na curva de luz obtida com o satélite CoRoT, o autor encontrou 462 frequências com amplitude significativa. A Figura 5.25 mostra as frequências encontradas com suas respectivas amplitudes. Dentre essas frequências, 33 foram observadas com espectroscopia das quais 28 tiveram os valores de $(1, \mathrm{~m})$ identificados.

Considerando as caixas de erro dos parâmetros físicos conhecidos de HD50844, calculamos modelos teóricos representativos da estrela. A Tabela 5.2 contém as características dos modelos calculados. Para os outros parâmetros físicos, utilizou-se os valores encontrados na Tabela 5.1. A Figura 5.26 mostra o diagrama HR contendo as trajetórias evolutivas computados e a caixa de erro dos parâmetros físicos determinados para a estrela HD50844.

Tabela 5.2 - Características dos modelos representativos da estrela $\delta$ Scuti HD50844.

\begin{tabular}{ccc}
\hline \hline Modelos & Massa $\left(M_{\odot}\right)$ & Velocidade Superficial \\
\hline modelo 1 & 2.00 & $60 \mathrm{~km} / \mathrm{s}$ \\
modelo 2 & 2.00 & $80 \mathrm{~km} / \mathrm{s}$ \\
modelo 3 & 2.00 & $100 \mathrm{~km} / \mathrm{s}$ \\
modelo 4 & 2.00 & $120 \mathrm{~km} / \mathrm{s}$ \\
modelo 5 & 2.20 & $60 \mathrm{~km} / \mathrm{s}$ \\
modelo 6 & 2.20 & $80 \mathrm{~km} / \mathrm{s}$ \\
modelo 7 & 2.20 & $100 \mathrm{~km} / \mathrm{s}$ \\
modelo 8 & 2.20 & $120 \mathrm{~km} / \mathrm{s}$ \\
modelo 9 & 2.40 & $60 \mathrm{~km} / \mathrm{s}$ \\
modelo 10 & 2.40 & $80 \mathrm{~km} / \mathrm{s}$ \\
modelo 11 & 2.40 & $100 \mathrm{~km} / \mathrm{s}$ \\
modelo 12 & 2.40 & $120 \mathrm{~km} / \mathrm{s}$ \\
\hline \hline
\end{tabular}




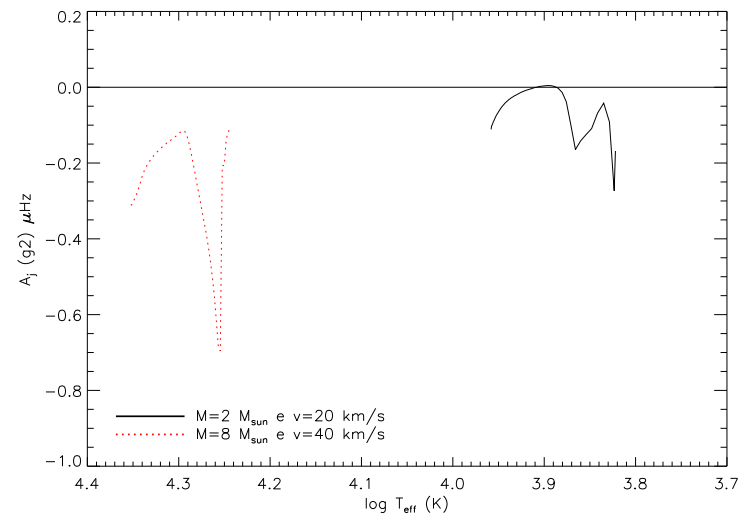

(a) modo g2

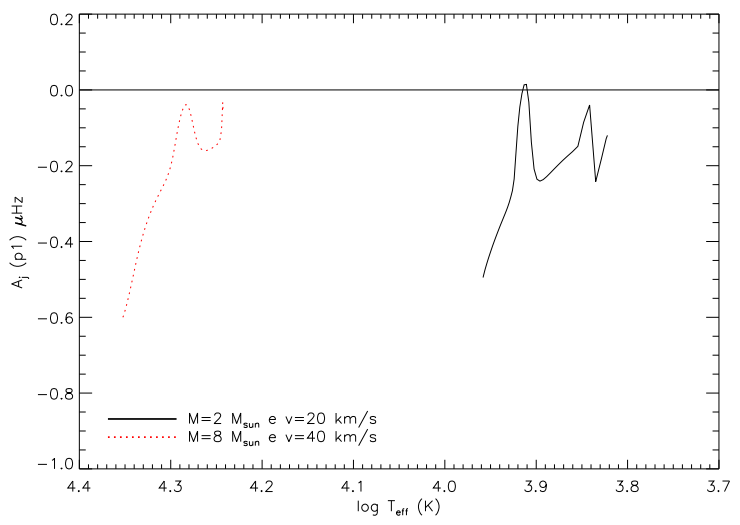

(c) modo p1

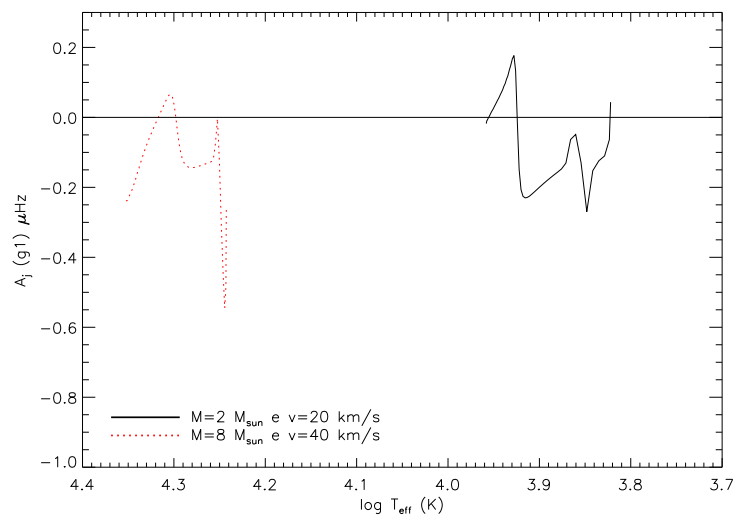

(b) modo g1

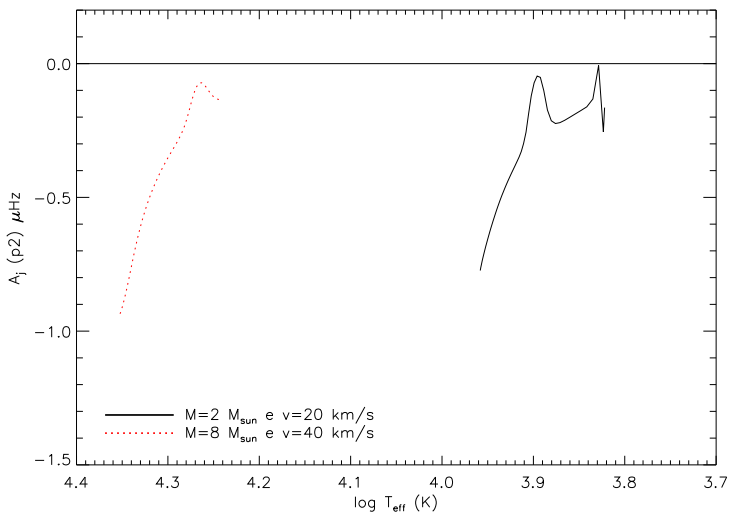

(d) modo p2

Figura 5.24: Comportamento da assimetria do splitting rotacional dos modos a) $g_{2}$, b) $g_{1}$, c) $p_{1}$ e d) $p_{2}$ em função da temperatura efetiva para as trajetórias evolutivas mostrando a influência da rotação para velocidades angulares similares. A velocidade angular correspondente à $v_{r}$ ot $=20 \mathrm{~km} / \mathrm{s}$ para a $2 M_{\odot}$ é igual a $2,3 \cdot 10^{-5} \mathrm{rad} / \mathrm{s}$ e à $v_{r}$ ot $=40 \mathrm{~km} / \mathrm{s}$ para $8 M_{\odot}$ é $2,1 \cdot 10^{-5} \mathrm{rad} / \mathrm{s}$. Note que a magnitude do splitting é da mesma ordem ao comparar modelos com velocidade angular próximas. 


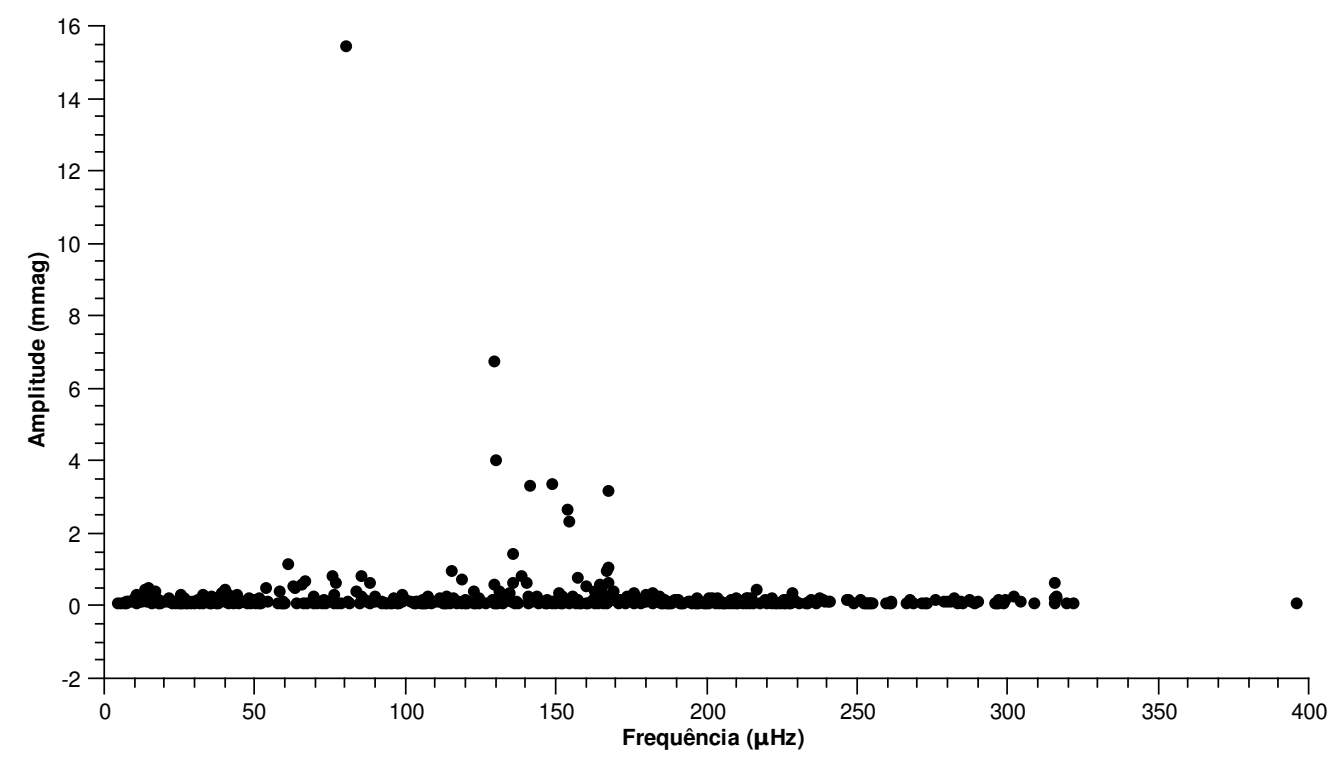

Figura 5.25: Espectro com as frequências obtidas por Poretti et al. (2009) da curva de luz observada com o CoRoT. Dentre essas frequências, somente 28 tiveram o valor $(1, \mathrm{~m})$ identificados através de espectroscopia.

Calculamos as frequências de oscilação para os modelos mencionados. A frequência do modo radial fundamental foi identificada no artigo com valor igual a 80,15370 $\mu \mathrm{Hz}$. Comparamos esse valor com os valores calculados na Figura 5.27. Nota-se que essa estrela provavelmente não constitui num caso científico clássico de estrela $\delta$ Scu porque ela esta localizada no final da sequência principal (TAMS). Esta estrela provavelmente pertence a classe de estrelas $\lambda$ Boo e está quase equator-on (Poretti et al., 2009).

O modelo que melhor representa o objeto é o de $2,2 M_{\odot}$ (Figuras 5.26 e 5.27), considerando uma metalicidade solar. A variação na velocidade de rotação é inexpressiva com relação à caixa de erro nos parâmetros estelares e, dessa forma, nada pode ser inferido sobre esse parâmetro.

Os tripletos dos modos $1=1$ calculados estão na Tabela 5.3 para os modelos com 2,2 $M_{\odot}$ dentro da caixa de erro, com 7,6 Gyrs. O comportamento do splitting rotacional e da sua assimetria são similares aos do modelo de 2,0 $M_{\odot}$ que podem ser visualizados nas Figuras 


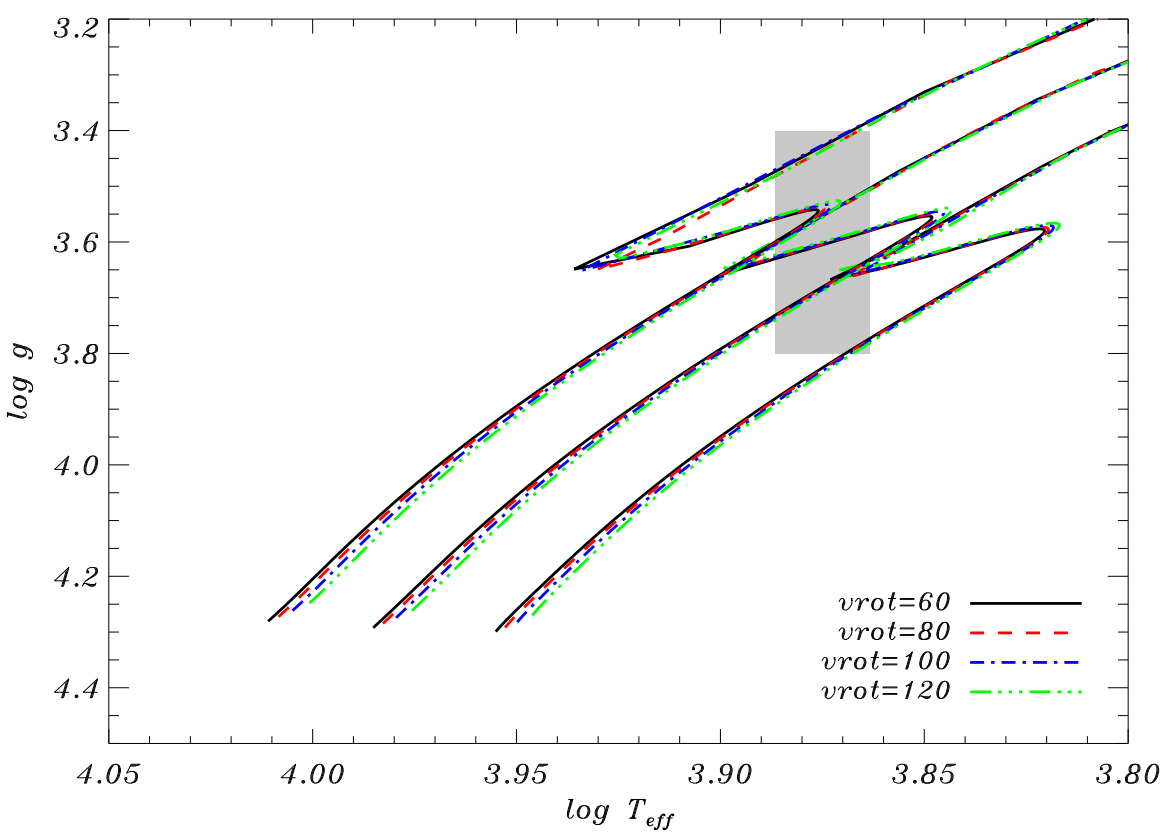

Figura 5.26: Diagrama HR contendo as trajetórias evolutivas dos 12 modelos representativos da estrela HD50844 calculados para as massas 2,0 a 2,4 $M_{\odot}$ (direita para a esquerda) com velocidade superficial de 60, 80, 100 e $120 \mathrm{~km} / \mathrm{s}$.

5.5 à 5.21. Comparando com o espectro das frequências encontradas na curva de luz obtida com o CoRoT (Figura 5.25), observamos diversas frequências com valores próximos aos valores da Tabela 5.3, por isso a identificação dos modos não é possível.

Tabela 5.3 - Frequência calculada para os modos $g_{2}, g_{1}, p_{1}$ e $p_{2}$ considerando os modelos de $2,2 M_{\odot}$ dentro da caixa de erro com idade de 7,6 Gyrs.

\begin{tabular}{cccc}
\hline \hline Modelo & Modo & $(1, \mathrm{~m})$ & Frequência $(\mu \mathrm{Hz})$ \\
\hline modelo 5 & modo $g_{2}$ & $(1,-1)$ & 86,103015 \\
modelo 5 & modo $g_{2}$ & $(1,0)$ & 83,403865 \\
modelo 5 & modo $g_{2}$ & $(1,1)$ & 79,345566 \\
modelo 5 & modo $g_{1}$ & $(1,-1)$ & 103,416969 \\
modelo 5 & modo $g_{1}$ & $(1,0)$ & 99,971483 \\
modelo 5 & modo $g_{1}$ & $(1,1)$ & 94,100032 \\
modelo 5 & modo $p_{1}$ & $(1,-1)$ & 111,290255 \\
modelo 5 & modo $p_{1}$ & $(1,0)$ & 108,540078 \\
modelo 5 & modo $p_{1}$ & $(1,1)$ & 104,237132 \\
Continua na próxima página... &
\end{tabular}


Tabela 5.3 - Continuação

\begin{tabular}{|c|c|c|c|}
\hline Modelo & Modo & $(\mathrm{l}, \mathrm{m})$ & Frequência $(\mu H z)$ \\
\hline modelo 5 & $\operatorname{modo} p_{2}$ & $(1,-1)$ & 136,733624 \\
\hline modelo 5 & $\operatorname{modo} p_{2}$ & $(1,0)$ & 134,303335 \\
\hline modelo 5 & $\operatorname{modo} p_{2}$ & $(1,1)$ & 129,797205 \\
\hline modelo 6 & modo $g_{2}$ & $(1,-1)$ & 86,947838 \\
\hline modelo 6 & modo $g_{2}$ & $(1,0)$ & 83,629146 \\
\hline modelo 6 & $\operatorname{modo} g_{2}$ & $(1,1)$ & 77,806597 \\
\hline modelo 6 & modo $g_{1}$ & $(1,-1)$ & 104,105042 \\
\hline modelo 6 & modo $g_{1}$ & $(1,0)$ & 99,984610 \\
\hline modelo 6 & $\operatorname{modo} g_{1}$ & $(1,1)$ & 91,621608 \\
\hline modelo 6 & $\operatorname{modo} p_{1}$ & $(1,-1)$ & 112,058122 \\
\hline modelo 6 & $\operatorname{modo} p_{1}$ & $(1,0)$ & 108,728513 \\
\hline modelo 6 & $\operatorname{modo} p_{1}$ & $(1,1)$ & 102,525886 \\
\hline modelo 6 & $\operatorname{modo} p_{2}$ & $(1,-1)$ & 137,283745 \\
\hline modelo 6 & $\operatorname{modo} p_{2}$ & $(1,0)$ & 134,506832 \\
\hline modelo 6 & $\operatorname{modo} p_{2}$ & $(1,1)$ & 127,889950 \\
\hline modelo 7 & $\operatorname{modo} g_{2}$ & $(1,-1)$ & 87,655908 \\
\hline modelo 7 & modo $g_{2}$ & $(1,0)$ & 83,890717 \\
\hline modelo 7 & $\operatorname{modo} g_{2}$ & $(1,1)$ & 76,060630 \\
\hline modelo 7 & $\operatorname{modo} g_{1}$ & $(1,-1)$ & 104,468253 \\
\hline modelo 7 & $\operatorname{modo} g_{1}$ & $(1,0)$ & 99,827208 \\
\hline modelo 7 & $\operatorname{modo} g_{1}$ & $(1,1)$ & 88,834045 \\
\hline modelo 7 & $\operatorname{modo} p_{1}$ & $(1,-1)$ & 112,601891 \\
\hline modelo 7 & $\operatorname{modo} p_{1}$ & $(1,0)$ & 108,923201 \\
\hline modelo 7 & $\operatorname{modo} p_{1}$ & $(1,1)$ & 100,544704 \\
\hline modelo 7 & $\operatorname{modo} p_{2}$ & $(1,-1)$ & 137,560590 \\
\hline modelo 7 & $\operatorname{modo} p_{2}$ & $(1,0)$ & 134,732574 \\
\hline modelo 7 & $\operatorname{modo} p_{2}$ & $(1,1)$ & 125,639241 \\
\hline modelo 8 & $\operatorname{modo} g_{2}$ & $(1,-1)$ & 88,142262 \\
\hline modelo 8 & modo $g_{2}$ & $(1,0)$ & 84,136122 \\
\hline modelo 8 & modo $g_{2}$ & $(1,1)$ & 74,134813 \\
\hline modelo 8 & modo $g_{1}$ & $(1,-1)$ & 104,561196 \\
\hline modelo 8 & $\operatorname{modo} g_{1}$ & $(1,0)$ & 99,489351 \\
\hline modelo 8 & $\operatorname{modo} g_{1}$ & $(1,1)$ & 85,987886 \\
\hline modelo 8 & $\operatorname{modo} p_{1}$ & $(1,-1)$ & 112,827054 \\
\hline
\end{tabular}


Tabela 5.3 - Continuação

\begin{tabular}{cccc}
\hline \hline Modelo & Modo & $(1, \mathrm{~m})$ & Frequência $(\mu H z)$ \\
\hline modelo 8 & modo $p_{1}$ & $(1,0)$ & 109,070412 \\
modelo 8 & modo $p_{1}$ & $(1,1)$ & 98,320563 \\
modelo 8 & modo $p_{2}$ & $(1,-1)$ & 137,456975 \\
modelo 8 & modo $p_{2}$ & $(1,0)$ & 134,909066 \\
modelo 8 & modo $p_{2}$ & $(1,1)$ & 123,060177 \\
\hline \hline
\end{tabular}




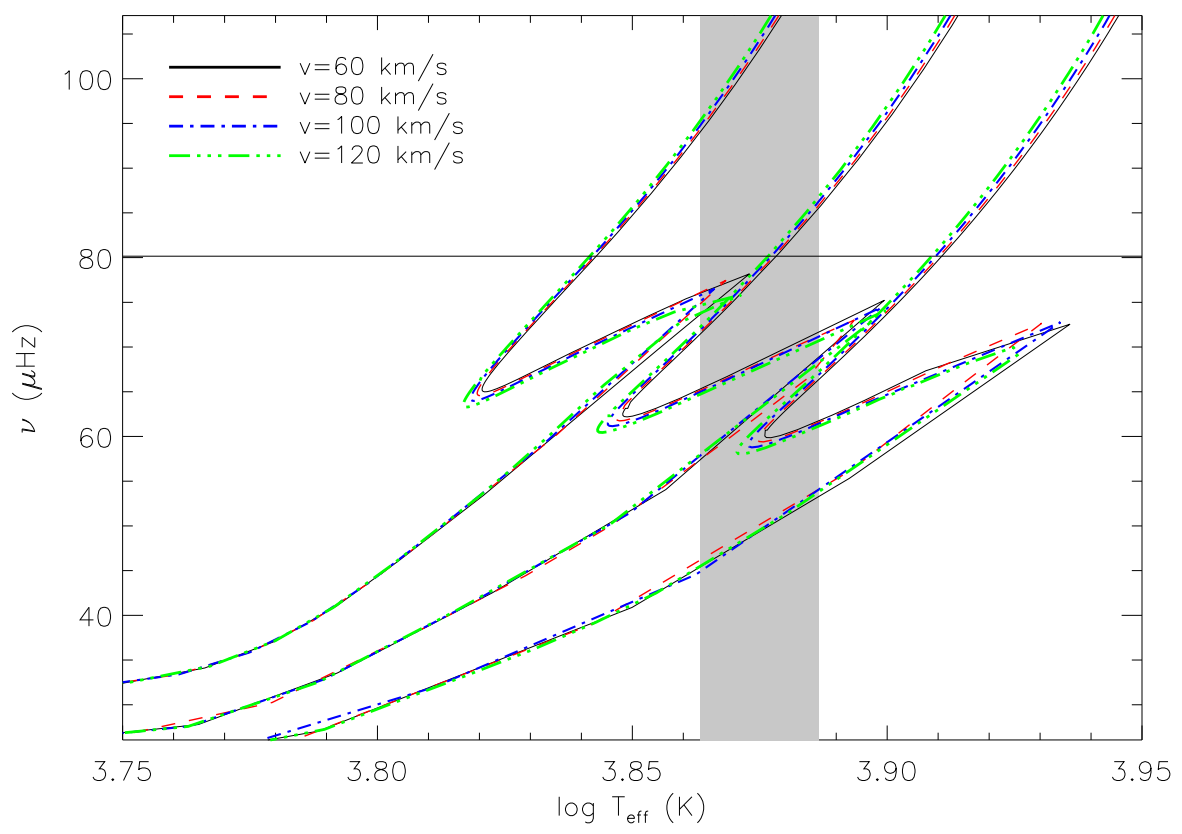

Figura 5.27: Frequência radial fundamental em função da temperatura efetiva para os 12 modelos representativos da estrela HD50844 calculados para as massas 2,0 a 2,4 $M_{\odot}$ (direita para a esquerda) com velocidade superficial de 60, 80, 100 e $120 \mathrm{~km} / \mathrm{s}$. A linha horizontal representa o valor da frequência $80,15370 \mu \mathrm{Hz}$ obtida da observação do CoRoT por Poretti et al. (2009). 


\section{Capítulo 6}

\section{Conclusões e Perspectivas}

A sismologia estelar é uma ferramenta poderosa para estudo da estrutura estelar. O objetivo deste trabalho foi estudar a dependência das frequências de oscilação estelar com a velocidade de rotação para modelos de massas intermediárias.

O splitting e a assimetria das frequências observadas nas estrelas apresentam um comportamento peculiar ao longo da evolução do objeto. Esses parâmetros mostram forte dependência com outros parâmetros físicos além da óbvia dependência com a velocidade de rotação estelar. A assimetria em particular apresenta momentos durante a evolução em que seu valor vai a zero independentemente da velocidade de rotação.

O comportamento da assimetria com a evolução da estrela na sequência principal pode ser utilizado como um importante método de diagnóstico de evolução. A observação de diferentes modos e suas respectivas assimetrias pode levar à determinação da idade da estrela com grande precisão, mostrando assim a importância do método sismológico no entendimento da evolução e estrutura estelar (cf., p. ex., Figura 5.15 e seção 5.4).

Modelos representativos da estrela HD50844 foram calculados a fim de comparar com os dados observacionais publicados por Poretti et al. (2009). Porém, a existência de um número muito grande de frequências sem identificação dos modos $(1, m)$ torna a comparação difícil. A não identificação de assimetrias não permitiu especular sobre a idade da estrela. Note que uma ausência de assimetria pode indicar que a estrela está numa determinada fase de evolução, onde a assimetria tende a se anular (cf. Figuras 5.15-5.21). Os resultados desse trabalho levaram a conclusão de que a estrela em questão está de fato na TAMS, como afirma o artigo.

O trabalho prosseguirá com o estudo do comportamento das frequências mudando 
outros parâmetros físicos como metalicidade, parâmetro de comprimento de mistura e overshooting. A análise da influência desses parâmetros pode ajudar na elucidação do desaparecimento da assimetria durante a evolução. Da mesma maneira, pretendemos comparar mais dados observacionais para estrelas de diferentes massa com nossos modelos. 


\section{Referências Bibliográficas}

Aerts C., Christensen-Dalsgaard J., Cunha M., Kurtz D. W., The Current Status of Asteroseismology, Sol. Phys., 2008, vol. 251, p. 3

Aizenman M., Smeyers P., Weigert A., Avoided Crossing of Modes of Non-radial Stellar Oscillations, A\&A, 1977, vol. 58, p. 41

Ando H., Osaki Y., Nonadiabatic nonradial oscillations - an application to the five-minute oscillation of the sun, PASJ, 1975, vol. 27, p. 581

Batchelor G. K., The Theory of Homogeneous Turbulence, 1953

Berthomieu G., Gonczi G., Graff P., Provost J., Rocca A., Low-frequency Gravity Modes of a Rotating Star, A\&A, 1978, vol. 70, p. 597

Böhm-Vitense E., Über die Wasserstoffkonvektionszone in Sternen verschiedener Effektivtemperaturen und Leuchtkräfte. Mit 5 Textabbildungen, ZAp, 1958, vol. 46, p. 108

Boisnard L., Auvergne M., CoRoT in Brief. In ESA Special Publication, vol. 1306 of ESA Special Publication, 2006, p. 19

Borucki W., Koch D., Basri G., et al. Finding Earth-size planets in the habitable zone: the Kepler Mission. In IAU Symposium, vol. 249 of IAU Symposium, 2008, p. 17

Breger M., Lenz P., Antoci V., et al. Detection of $75+$ pulsation frequencies in the $\delta$ Scuti star FG Virginis, A\&A, 2005, vol. 435, p. 955

Canuto V. M., Mazzitelli I., Stellar turbulent convection - A new model and applications, ApJ, 1991, vol. 370, p. 295 
Christensen-Dalsgaard J., Helioseismology, Reviews of Modern Physics, 2002, vol. 74, p. 1073

Christensen-Dalsgaard J., Stellar Oscillations. 5th ed., 2003, 268 p.

Christensen-Dalsgaard J., Seismological challenges for stellar structure, ArXiv e-prints, 2010

Christensen-Dalsgaard J., Daeppen W., Solar oscillations and the equation of state, A\&A Rev., 1992, vol. 4, p. 267

Christensen-Dalsgaard J., Gough D. O., On the interpretation of five-minute oscillations in solar spectrum line shifts, MNRAS, 1982, vol. 198, p. 141

CNES, 2011 CoRoT http://corot.oamp.fr/index.html

Cowling T. G., The non-radial oscillations of polytropic stars, MNRAS, 1941, vol. 101, p. 367

Cowling T. G., Newing R. A., The Oscillations of a Rotating Star., ApJ, 1949, vol. 109, p. 149

Cox J. P., Giuli R. T., Principles of stellar structure, 1968

CSA, 2011 MOST: Canada's First Space Telescope http://www.astro .ubc.ca/MOST/

Cunha M. S., Aerts C., Christensen-Dalsgaard J., Baglin A., et al. Asteroseismology and interferometry, A\&A Rev., 2007, vol. 14, p. 217

Cunha M. S., Théado S., Vauclair S., Excitation of the oscillations in roAp stars: Magnetic fields, diffusion, and winds. In The A-Star Puzzle , vol. 224 of IAU Symposium, 2004, p. 359

Deubner F., Observations of low wavenumber nonradial eigenmodes of the sun, A\&A, 1975, vol. 44 , p. 371

Domiciano de Souza A., Kervella P., Jankov S., Abe L., Vakili F., di Folco E., Paresce F., The spinning-top Be star Achernar from VLTI-VINCI, A\&A, 2003, vol. 407, p. L47 
Eggleton P. P., Faulkner J., Flannery B. P., An Approximate Equation of State for Stellar Material, A\&A, 1973, vol. 23, p. 325

Favata F., Roxburgh I., Christensen-Dalsgaard J., Eddington: A mission to map stellar evolution through oscillations and to find habitable planets, Assessment Study Report/ESA, 2000

Gough D., Solar inverse theory. In Solar Seismology from Space , 1984, p. 49

Kepler S. O., Giovannini O., Wood M. A., et al. Whole Earth Telescope Observations of the DAV White Dwarf G226-29, ApJ, 1995, vol. 447, p. 874

Kippenhahn R., Weigert A., Stellar Structure and Evolution, 1990

Koch D. G., Borucki W. J., Basri et al. Kepler Mission Design, Realized Photometric Performance, and Early Science, ApJ, 2010, vol. 713, p. L79

Kumar P., Franklin J., Goldreich P., Distribution functions for the time-averaged energies of stochastically excited solar p-modes, ApJ, 1988, vol. 328, p. 879

Leavitt H. S., 1777 variables in the Magellanic Clouds, Annals of Harvard College Observatory, 1908 , vol. 60 , p. 87

Ledoux P., The Nonradial Oscillations of Gaseous Stars and the Problem of Beta Canis Majoris., ApJ, 1951, vol. 114, p. 373

Leighton R. B., Noyes R. W., Simon G. W., Velocity Fields in the Solar Atmosphere. I. Preliminary Report., ApJ, 1962, vol. 135, p. 474

Minh F. T., Léon L., Numerical solution of stellar nonradial oscillations: the Galerkin and B-Splines method. In Particle Physics and Astrophysics, vol. 458 of Lecture Notes in Physics, Berlin Springer Verlag, 1995, p. 219

Morel P., CESAM: A code for stellar evolution calculations, A\&AS, 1997, vol. 124, p. 597 NASA, 2011 Kepler: A Search for Habitable Planets http://www.nasa.gov/mission_pages/kepler/main/index.html 
Osaki J., Nonradial oscillations of a 10 solar mass star in the main-sequence stage, PASJ, 1975, vol. 27, p. 237

Osaki Y., Non-Radial Oscillations and the Beta Canis Majoris Phenomenon, PASJ, 1971, vol. 23 , p. 485

Padmanabhan T., Theoretical Astrophysics, Volume 2: Stars and Stellar Systems, 2001

Pekeris C. L., Nonradial Oscillations of Stars., ApJ, 1938, vol. 88, p. 189

Poretti E., Michel E., Garrido R. e. a., HD 50844: a new look at $\delta$ Scuti stars from CoRoT space photometry, A\&A, 2009, vol. 506, p. 85

Rodríguez E., Breger M., delta Scuti and related stars: Analysis of the R00 Catalogue, A\&A, 2001, vol. 366, p. 178

Schaller G., Schaerer D., Meynet G., Maeder A., New grids of stellar models from 0.8 to 120 solar masses at $\mathrm{Z}=0.020$ and $\mathrm{Z}=0.001$, A\&AS, 1992, vol. 96, p. 269

Scuflaire R., The Non Radial Oscillations of Condensed Polytropes, A\&A, 1974, vol. 36, p. 107

Shapley H., On the Nature and Cause of Cepheid Variation, ApJ, 1914, vol. 40, p. 448

Smolec R., Moskalik P., Amplitude saturation in $\beta$ Cephei models, MNRAS, 2007, vol. 377, p. 645

Soufi F., Goupil M. J., Dziembowski W. A., Effects of moderate rotation on stellar pulsation. I. Third order perturbation formalism, A\&A, 1998, vol. 334, p. 911

Stankov A., Handler G., Catalog of Galactic $\beta$ Cephei Stars, ApJS, 2005, vol. 158, p. 193

Suárez J. C., Seismology of rotating stars. Application to delta Scuti stars, Observatoire de Paris, 2002, Tese de Doutorado

Suárez J. C., Goupil M. J., filou oscillation code, Ap\&SS, 2008, vol. 316, p. 155

Suárez J. C., Goupil M. J., Morel P., Effects of moderately fast shellular rotation on adiabatic oscillations, A\&A, 2006, vol. 449, p. 673 
Suárez J. C., Moya A., Amado P. J., Martín-Ruiz S., Rodríguez-López C., Garrido R., Seismology of $\beta$ Cephei Stars: Differentially Rotating Models for Interpreting the Oscillation Spectrum of $\nu$ Eridani, ApJ, 2009, vol. 690, p. 1401

Tassoul J., Stellar Rotation, 2000

Tassoul M., Asymptotic approximations for stellar nonradial pulsations, ApJS, 1980, vol. 43 , p. 469

Théado S., Vauclair S., Castro M., Charpinet S., Dolez N., Asteroseismic tests of element diffusion in solar type stars, A\&A, 2005, vol. 437, p. 553

Unno W., Osaki Y., Ando H., Saio H., Shibahashi H., Nonradial oscillations of stars, 1989

Vauclair S., Théado S., Asteroseismic signatures of helium gradients in main-sequence A stars; application to the roAp star HD 60435, A\&A, 2004, vol. 425, p. 179

Walker G., Matthews J., Kuschnig R., et al. The MOST Asteroseismology Mission: Ultraprecise Photometry from Space, PASP, 2003, vol. 115, p. 1023

Wolff C. L., Some simple properties of stellar pulsation modes, ApJ, 1979, vol. 227, p. 943

Zahn J., Convective penetration in stellar interiors, A\&A, 1991, vol. 252, p. 179 